\title{
Diffusion Profile for Random Band Matrices: a Short Proof
}

\author{
Yukun $\mathrm{He}^{*} \quad$ Matteo Marcozzi ${ }^{\dagger}$
}

September 11, 2019

\begin{abstract}
Let $H$ be a Hermitian random matrix whose entries $H_{x y}$ are independent, centred random variables with variances $S_{x y}=\mathbb{E}\left|H_{x y}\right|^{2}$, where $x, y \in(\mathbb{Z} / L \mathbb{Z})^{d}$ and $d \geqslant 1$. The variance $S_{x y}$ is negligible if $|x-y|$ is bigger than the band width $W$.

For $d=1$ we prove that if $L \ll W^{1+\frac{2}{7}}$ then the eigenvectors of $H$ are delocalized and that an averaged version of $\left|G_{x y}(z)\right|^{2}$ exhibits a diffusive behaviour, where $G(z)=(H-z)^{-1}$ is the resolvent of $H$. This improves the previous assumption $L \ll W^{1+\frac{1}{4}}$ of [9]. In higher dimensions $d \geqslant 2$, we obtain similar results that improve the corresponding ones from [9]. Our results hold for general variance profiles $S_{x y}$ and distributions of the entries $H_{x y}$.

The proof is considerably simpler and shorter than that of [7,9]. It relies on a detailed Fourier space analysis combined with isotropic estimates for the fluctuating error terms. It is completely self-contained and avoids the intricate fluctuation averaging machinery from [7].
\end{abstract}

\section{Introduction}

Given a large finite graph $\Gamma$, random band matrices $H=\left(H_{x y}\right)_{x, y \in \Gamma}$ are matrices whose entries $H_{x y}$ are independent and centred random variables and the variance $S_{x y}:=\mathbb{E}\left|H_{x y}\right|^{2}$ typically decays with the distance on a characteristic length scale $W$, called the band width of $H$.

This name is due to the simplest one-dimensional model where $\Gamma=\{1,2, \ldots, N\}$ and $H_{x y}=0$ if $|x-y| \geqslant W$, where $1 \leqslant W \leqslant L$. As an example of higher-dimensional models, one can take $\Gamma$ to be the box of linear size $L$ in $\mathbb{Z}^{d}$, so that the dimension of the matrix is $N=L^{d}$. For a more general and extensive presentation of random band matrix models, we refer to [17].

From the physics view point, random band matrices turn out to be very useful to study the disordered systems. In fact, it is conjectured that, depending on the level of energy and disorder strength, all these systems belong to two universality classes: in the strong disorder regime (as for the random Schrödinger operator models such as the Anderson model [1]), the eigenfunctions are localized and the local spectral statistics are Poisson, while in the weak disorder regime (as for the mean-field models such as Wigner matrices [18]), the eigenfunctions are delocalized and the local statistics are those of a mean-field Gaussian matrix ensemble.

As $W$ varies, random band matrices interpolate between these two classes: in particular, we recover the Wigner matrices by setting $W=N$ and all variances equal, while for $W=O(1)$ we essentially obtain the Anderson model. The delocalization property is expressed in the term of the localization length $\ell$, which, in the framework of random matrices, describes the typical length scale of the eigenvectors of $H$ : if the localization length is comparable with the system size, $\ell \sim L$, the system is delocalized and it is localized otherwise. The direct physical interpretation of the delocalization is that delocalized systems describe electric conductors, while localized systems insulators. Therefore, random band matrices represent a good model to investigate the Anderson metal-insulator phase transition.

According to nonrigorous results [11], for random band matrices the localization length is expected to be $\ell \sim W^{2}$ in $d=1$, exponentially growing in $W$ in $d=2$, and $\ell \sim L$ in $d \geqslant 3$, i.e. the system is delocalized. Notice that the claim that $\ell \sim W^{2}$ in $d=1$ is equivalent to have the delocalization of the eigenvectors for $W \geqslant L^{\frac{1}{2}}$ : this is the formulation used in the abstract. For more details on these conjectures, see [15-17].

\footnotetext{
*University of Zürich, Institute of Mathematics, yukun.he@math.uzh.ch.

$\dagger$ University of Geneva, Section of Mathematics, matteo.marcozzi@unige.ch.
} 
Up to now, only lower and upper bounds have been rigorously established for $\ell$. Since the Anderson transition can be studied via random matrices directly in $d=1$ by varying $W$ in the interval $1 \leqslant W \leqslant L$, here we focus on the results in the one-dimensional setting, but analogous theorems were proved for higher dimensions.

Schenker [15] showed that $\ell \leqslant W^{8}$, uniformly in the system size. The lower bound $\ell \geqslant W$ was proved in [10] by using a self-consistent equation for the diagonal matrix entries $G_{x x}$ of the resolvent (or Green function) $G=G(z)=(H-z)^{-1}, z=E+\mathrm{i} \eta \in \mathbb{C}$. In a series of papers Erdős and collaborators improved this lower bound: in $[5,6]$ it was proved that $\ell \geqslant W^{1+\frac{1}{6}}$ by using diagrammatic perturbation theory and then in [9] the authors showed that $\ell \geqslant W^{1+\frac{1}{4}}$ by employing a self consistent equation for an averaged version of $G$ and the so called fluctuation averaging estimates [7].

In $[5,6]$ the unitary time evolution, $\mathrm{e}^{\mathrm{i} t H}$, was analysed and it was shown to behave diffusively on the spatial scale $W$, i.e. the typical propagation distance is $\sqrt{t} W$. From this, one deduces that a (superposition of) random walk with step size of order $W$ is responsible for the delocalization of random band matrices. The barrier at $W^{1+\frac{1}{6}}$ is due to the fact, in order to see that the localization length $\ell$ is greater than the naive size $W$, a control of the random walk for large times is needed, but the diagrammatic expansion used in $[5,6]$ allows to control the time evolution only up to time $t \ll W^{1 / 3}$. Hence, the delocalization occurs on the scale $\sqrt{t} W=W^{1+\frac{1}{6}}$.

In $[7,9]$ the same result was translated in term of the resolvent $G$ : as explained in Remark 2.7 in [9], controlling $\mathrm{e}^{\mathrm{i} t H}$ up to $t \ll W^{\nu}$ for some exponent $\nu>0$ is equivalent to controlling $G(z)$ for $\eta \gg W^{-\nu}$. In $[7,9]$ the authors managed to overcome the technical barrier of $[5,6]$ and they proved the diffusion behaviour of $G(z)$ when $\eta \gg W^{-1 / 2}$.

We emphasize that the delocalization results $[5-7,9]$ hold for general variance profiles $S_{x y}$ and distributions of the entries $H_{x y}$. Our results hold under similarly general assumptions.

In this paper we improve the results of $[7,9]$ : we prove that $\ell \gg W^{1+\frac{2}{7}}$ and that $G(z)$ exhibits a diffusive behaviour for $\eta \gg W^{-4 / 7}$. As in [9], the main object of interest is the matrix $T$, whose entries are local averages of $\left|G_{x y}\right|^{2}$ :

$$
T_{x y}:=\sum_{i} S_{x i}\left|G_{i y}\right|^{2}
$$

The band structure of $H$ is obtained by assuming that $S_{x y}:=\mathbb{E}\left|H_{x y}\right|^{2} \approx W^{-d} f((i-j) / W)$ where $f$ is a symmetric probability density on $\mathbb{R}^{d}$. Note that in this way $S$ is translation invariant.

We will show that $T$ satisfies a self-consistent equation of the form

$$
T_{x y}=|\mathfrak{m}|^{2} \sum_{i} S_{x i} T_{i y}+|\mathfrak{m}|^{2} S_{x y}+\mathcal{E}_{x y}
$$

where $\mathfrak{m} \equiv \mathfrak{m}(z)$ is an explicit function of the spectral parameter $z \in \mathbb{C}$ and $\mathcal{E}$ is an error term.

Translation invariance of $S$ implies that its Fourier transform $\widehat{s}(p)$ for $|p| \ll W^{-1}$ reads

$$
\widehat{s}(p) \approx 1-W^{2}(p \cdot D p)+\cdots,
$$

where $D$ is the matrix of second moments of $f$ (see (2.25)).

Neglecting the error term $\mathcal{E}$, we get from (1.1) that

$$
T \approx \frac{|\mathfrak{m}|^{2} S}{1-|\mathfrak{m}|^{2} S}
$$

By using $|\mathfrak{m}|^{2}=1-\alpha \eta+O\left(\eta^{2}\right)$ (see (3.6) below), where

$$
\alpha \equiv \alpha(E):=\frac{2}{\sqrt{4-E^{2}}} \quad(E=\operatorname{Re} z)
$$

we obtain that the Fourier transform of $T$ is approximately given by

$$
\frac{\alpha^{-1}}{\eta+W^{2}\left(p \cdot D_{\text {eff }} p\right)} \quad \text { where } \quad D_{\text {eff }}:=\frac{D}{\alpha},
$$


for $|p| \ll W^{-1}$ and $\eta \ll 1$. This corresponds to the diffusion approximation on scales larger than $W$ with an effective diffusion constant $D_{\text {eff }}$. As in [7,9], the main work of the proof is to estimate the error term $\mathcal{E}_{x y}$ from (1.1), in order to make the approximation (1.3) rigorous.

The crucial difference between our approach and that of [9] is how this error term is estimated. In [9] it is bounded by the fluctuation averaging estimates relying on very intricate expansions derived using the Schur's complement formula [7]. In contrast, in this paper we first perform a careful analysis of the error term in Fourier space, and then apply isotropic error estimates to the resulting Fourier coefficients. Here, isotropic refers to generalized matrix entries $\langle\mathbf{v}, \mathcal{E} \mathbf{w}\rangle$ where the vectors $\mathbf{v}, \mathbf{w}$ do not lie in the direction of the standard coordinate axes. A trivial but essential observation behind our proof is that the Fourier basis is completely delocalized. The isotropic error estimates are derived using the cumulant expansion formula (see Lemma 3.2 below) in the formulation given in $[12,13]$. We do not use Schur's complement formula at all. Thus, apart from sharing the basic starting point and self-consistent equation (1.1) with [7,9], our proof differs fundamentally from that of [7,9]. For more details on the proof strategy, see Section 4. We would also like to emphasis that our proof is considerably simpler than that of [7,9]: the arguments in [9] need [7] as an input, and they are altogether 120 pages; on the other hand, our proof is completely self-contained.

More recently, the series of works [2,3,19] further improves the result of this paper. For $W \gg L^{\frac{3}{4}}$, it was proved that the $L^{\infty}$-norm of the bulk eigenvectors of $H$ are all simultaneously bounded by $N^{-\frac{1}{2}+\varepsilon}$ with overwhelming probability. The authors also prove bulk universality under the same condition. The proof uses a strong (high probability) version of the quantum unique ergodicity property of random matrices, as well as estimates on the "generalized resolvent" of band matrices. The later is obtained by the intricate fluctuation averaging machinery similar to [10], but the estimates are done much more carefully. As a result, [2,3,19] are also more lengthy and complicated than this paper.

Acknowledgments. We thank Antti Knowles, who motivated us to study this problem, for useful discussions and suggestions on the topic. This project has received funding from the European Research Council (ERC) under the European Unions Horizon 2020 research and innovation programme (grant agreement No. 715539_RandMat) and the Swiss National Science Foundation (SNF).

\section{The main results}

Let $f: \mathbb{R}^{d} \rightarrow \mathbb{R}$ be a smooth and symmetric probability density for some fixed $d \geqslant 1$. Let $L, W \in \mathbb{N}$ such that

$$
L^{\delta} \leqslant W \leqslant L
$$

for some fixed $\delta>0$. We set $\mathbb{T}_{L}^{d}:=[-L / 2, L / 2)^{d} \cap \mathbb{Z}^{d}$ to be the $d$-dimensional discrete torus, so that $\mathbb{T}_{L}^{d}$ has $N:=L^{d}$ lattice points. For the following we fix an (arbitrary) ordering of $\mathbb{T}_{L}^{d}$, which allows us to identify it with $\{1, \ldots, N\}$. Let the canonical representative of $i \in \mathbb{Z}^{d}$ be

$$
[i]_{L}:=\left(i+L \mathbb{Z}^{d}\right) \cap \mathbb{T}_{L}^{d}
$$

and the periodic distance $|i|_{L}:=\left|[i]_{L}\right|$, where $|\cdot|$ denotes Euclidean distance in $\mathbb{R}^{d}$.

Moreover, define the $N \times N$ matrix $S(L, W) \equiv S=\left(S_{i j}: i, j \in \mathbb{T}_{L}^{d}\right)$ through

$$
S_{i j}:=\frac{1}{Z_{L, W}} f\left(\frac{[i-j]_{L}}{W}\right)
$$

where $Z_{L, W}$ is a normalization constant such that for all $i \in \mathbb{T}_{L}^{d}$

$$
\sum_{j} S_{i j}=1
$$

i.e. $S$ is a stochastic matrix. Here we adopted the convention that summations are always over the set $\mathbb{T}_{L}^{d}$, unless specified otherwise. The symmetry of $f$ implies that $S$ is also symmetric: $S_{i j}=S_{j i}$. 
Let $\left(\zeta_{i j}: i \leqslant j\right)$, where $i, j \in \mathbb{T}_{L}^{d}$, be a family of independent, complex-valued, centred random variables $\zeta_{i j} \equiv \zeta_{i j}^{(N)}$ satisfying

$$
\mathbb{E} \zeta_{i j}=0, \quad \mathbb{E}\left|\zeta_{i j}\right|^{2}=1, \quad \zeta_{i i} \in \mathbb{R}, \quad \zeta_{i j}:=\bar{\zeta}_{j i} \text { for } i>j .
$$

The band matrix $H=\left(H_{i j}\right)_{i, j \in \mathbb{T}_{L}^{d}}$ is defined as

$$
H_{i j}:=\left(S_{i j}\right)^{1 / 2} \zeta_{i j}
$$

By definition we have $H=H^{*}$ and $\mathbb{E}\left|H_{i j}\right|^{2}=S_{i j}$. Moreover, we assume that the random variables $\zeta_{i j}$ have finite moments, uniformly in $N, i$, and $j$, in the sense that for all $p \in \mathbb{N}$ there is a constant $\mu_{p}$ such that

$$
\mathbb{E}\left|\zeta_{i j}\right|^{p} \leqslant \mu_{p}
$$

for all $N, i$, and $j$. Furthermore, we set the parameter

$$
M \equiv M_{N}:=\frac{1}{\max _{i, j} S_{i j}}
$$

Note that $M=\left(W^{d}+O\left(W^{d-1}\right)\right) /\|f\|_{\infty}$ since the definition of $S$ implies that $Z_{N, W}=W^{d}+O\left(W^{d-1}\right)$. Here we used the usual $O(\cdot)$ notation. Furthermore, we will write $O_{\alpha}(\cdot)$ if the implicit constant depends on the parameter $\alpha$ which can never depend on $N$.

The following definition introduces a notion of a high-probability bound that will be used throughout the whole paper.

Definition 2.1 (Stochastic domination). Let

$$
X=\left(X^{(N)}(u): N \in \mathbb{N}, u \in U^{(N)}\right), \quad Y=\left(Y^{(N)}(u): N \in \mathbb{N}, u \in U^{(N)}\right)
$$

be two families of random variables, where the $Y^{(N)}(u)$ are nonnegative and $U^{(N)}$ is a possibly $N$-dependent parameter set. We say that $X$ is stochastically dominated by $Y$, uniformly in $u$, if for all $\varepsilon>0$ and $D>0$ we have

$$
\sup _{u \in U^{(N)}} \mathbb{P}\left[\left|X^{(N)}(u)\right|>N^{\varepsilon} Y^{(N)}(u)\right] \leqslant N^{-D}
$$

for large enough $N \geqslant N_{0}(\varepsilon, D)$. Unless stated otherwise, throughout this paper the stochastic domination will always be uniform in all parameters apart from the parameter $\delta$ in (2.1) and the sequence of constants $\mu_{p}$ in (2.6); thus, $N_{0}(\varepsilon, D)$ also depends on $\delta$ and $\mu_{p}$. If $X$ is stochastically dominated by $Y$, uniformly in $u$, we use the equivalent notations

$$
X \prec Y \quad \text { and } \quad X=O_{\prec}(Y) .
$$

As stated in Lemma 3.1 below, the relation $\prec$ satisfies the familiar algebraic rules of order relations. Moreover, note that for deterministic $X$ and $Y, X=O_{\prec}(Y)$ implies $X=O_{\varepsilon}\left(N^{\varepsilon} Y\right)$ for any $\varepsilon>0$.

We note that, if (2.6) only holds for some large but fixed $p$, Definition 2.1 should be slightly modified so that our results would still remain true, even though in a slightly weaker sense. In fact, in this case the exponents $\varepsilon$ and $D$ in the definition of $\prec$ must depend on $p$, therefore one should keep track of the $p$ dependencies in all the stochastic domination bounds involved in the proof.

We use the spectral parameter $z=E+\mathrm{i} \eta \in \mathbb{C}$ with $\eta:=\operatorname{Im} z>0$ and we fix two arbitrary (small) global constants $\gamma>0$ and $\kappa>0$. All of our estimates will depend on $\kappa$ and $\gamma$, and we shall often omit the explicit mention of this dependence. We set

$$
\mathbf{S} \equiv \mathbf{S}^{(N)}(\kappa, \gamma):=\left\{E+\mathrm{i} \eta:-2+\kappa \leqslant E \leqslant 2-\kappa, M^{-1+\gamma} \leqslant \eta \leqslant 10\right\} .
$$

and we shall always assume that $z \in \mathbf{S}(\kappa, \gamma)$.

We will use the Stieltjes transform of Wigner's semicircle law, which is defined by

$$
\mathfrak{m}(z):=\frac{1}{2 \pi} \int_{-2}^{2} \frac{\sqrt{4-\xi^{2}}}{\xi-z} \mathrm{~d} \xi
$$


and is characterized by the unique solution of

$$
\mathfrak{m}(z)+\frac{1}{\mathfrak{m}(z)}+z=0
$$

with $\operatorname{Im} \mathfrak{m}(z)>0$ for $\operatorname{Im} z>0$, i.e.

$$
\mathfrak{m}(z)=\frac{-z+\sqrt{z^{2}-4}}{2}
$$

We define the resolvent of $H$ through

$$
G \equiv G(z):=(H-z)^{-1}
$$

and denote its entries by $G_{i j}(z)$.

Moreover, throughout the paper $C$ and $c$ will denote a generic large and small positive constant respectively, which may depend on some fixed parameters and whose value may change from one expression to the next. Given two positive quantities $A_{N}$ and $B_{N}$, the notation $A_{N} \asymp B_{N}$ means $c A_{N} \leqslant B_{N} \leqslant C A_{N}$, while $A_{N} \ll B_{N}$ means that there exists a constant $c>0$ such that $A_{N} \leqslant N^{-c} B_{N}$; we also use $A_{N} \gg B_{N}$ to denote $B_{N} \ll A_{N}$. We also set $\langle x\rangle:=\sqrt{1+|x|^{2}}$. Notice that we will often drop the $z$ dependence from the notation, even though most quantities in this paper depend on it.

Finally, we assume the following decay condition on $f$ (and therefore on $S$ )

$$
|f(x)| \leqslant C_{n}\langle x\rangle^{-n} \quad \text { for all } n \in \mathbb{N} .
$$

We are now ready to state our main theorems in $d=1$. The analogous results for $d>1$ are discussed in Section 9 .

2.1. Local semicircle law and delocalization. We introduce the control parameter $\Phi$ through

$$
\Phi^{2}:=\frac{1}{L \eta}+\frac{1}{W \sqrt{\eta}} .
$$

Our first theorem is a local semicircle law for the resolvent entries: more precisely we get a bound for the random variable

$$
\Lambda(z):=\max _{x, y}\left|G_{x y}(z)-\delta_{x y} \mathfrak{m}(z)\right| .
$$

Theorem 2.2 (Local semicircle law). Assume (2.12) and that

$$
L \ll W^{1+\frac{1}{3}}, \quad \eta \gg \frac{L^{3 / 2}}{W^{5 / 2}} .
$$

Then for $z \in \mathbf{S}$ we have

$$
\Lambda^{2} \prec \Phi^{2} .
$$

This improves Theorem 2.2 in [9] where the assumptions are $L \ll W^{1+\frac{1}{4}}$ and $\eta \gg \frac{L^{2}}{W^{3}}=\frac{L^{3 / 2}}{W^{5 / 2}} \frac{L^{1 / 2}}{W^{1 / 2}}$. Note also that, as one can see from (2.26) and (2.20) below, (2.15) is optimal.

We observe that by spectral decomposition of $G$ one gets that for any $d$

$$
\frac{1}{N^{2}} \sum_{x, y}\left|G_{x y}\right|^{2}=\frac{1}{N^{2}} \operatorname{Tr} G^{*} G=\frac{1}{N \eta} \operatorname{Im} \frac{\operatorname{Tr} G}{N}=\frac{\operatorname{Im} m}{N \eta}+O_{\prec}\left(\frac{\Lambda}{N \eta}\right) .
$$

Furthermore, from (2.13) we have $\Phi^{2} \leqslant C(L \eta)^{-1}$ for $\eta \leqslant(W / L)^{2}$, thus (2.15) shows that all off-diagonal entries of $G$ have a magnitude comparable with the average of their magnitudes computed in (2.16): in other words, $\left|G_{x y}\right|^{2}$ is essentially flat. In this case we say that the resolvent is completely delocalized and this implies that the eigenvectors of $H$ are delocalized.

More precisely, let us denote the eigenvalues of $H$ by $\lambda_{1} \leqslant \lambda_{2} \leqslant \cdots \leqslant \lambda_{N}$, and the associated normalized eigenvectors by $\mathbf{u}_{1}, \mathbf{u}_{2} \ldots, \mathbf{u}_{N}$. We use the notation $\mathbf{u}_{\alpha}=\left(u_{\alpha}(x)\right)_{x=1}^{N}$. We shall only consider eigenvectors associated with eigenvalues lying in the interval

$$
\mathcal{I}:=[-2+\kappa, 2-\kappa],
$$


where $\kappa>0$ is fixed. For $\ell \equiv \ell(N)$, define the characteristic function $P_{x, \ell}$ projecting onto the complement of the $\ell$-neighborhood of $x$,

$$
P_{x, \ell}(y):=\mathbf{1}(|x-y| \geqslant \ell) .
$$

Let $\varepsilon>0$ and define the random subset of eigenvector indices through

$$
\mathcal{A}_{\varepsilon, \ell}:=\left\{\alpha: \lambda_{\alpha} \in \mathcal{I}, \sum_{x}\left|u_{\alpha}(x)\right|\left\|P_{x, \ell} \mathbf{u}_{\alpha}\right\| \leqslant \varepsilon\right\}
$$

which indexes the set of eigenvectors localized on scale $\ell$ up to an error $\varepsilon$.

As stated in [9, Proposition 7.1], given the interval the condition for the eigenvector delocalization is that

$$
\sup _{E \in \mathcal{I}}\left|G_{x y}(E+\mathrm{i} \eta)\right|^{2} \prec \frac{1}{N \eta}+\delta_{x y}
$$

for $\eta$ such that $M^{-1+\gamma} \leqslant \eta \ll 1$. Hence by choosing $\eta$ such that $L^{3 / 2} W^{-5 / 2} \ll \eta \leqslant W^{2} L^{-2}$, (2.17) and Theorem 2.2 imply the following corollary.

Corollary 2.3 (Eigenvector delocalization). Assume (2.12) and $L \ll W^{1+\frac{2}{7}}$. Then the eigenvectors of $H$ are completely delocalized in the sense that for any $\ell \ll N$ and fixed $\varepsilon>0$, we have

$$
\frac{\left|A_{\varepsilon, \ell}\right|}{N} \leqslant C \sqrt{\varepsilon}+O_{\prec}\left(N^{-c}\right) .
$$

This improves [9, Corollary 2.4], where the condition is $L \ll W^{1+\frac{1}{4}}$.

2.2. Diffusion profile. Set $\mathbb{T}_{L}^{1} \equiv \mathbb{T}_{L}$. In the previous section we saw that for $\eta \leqslant(W / L)^{2}$ the profile of $\left|G_{x y}\right|^{2}$ is essentially flat, while we will see that for $\eta \geqslant(W / L)^{2}$, an averaged version of $\left|G_{x y}\right|^{2}$ is well approximated by a diffusion profile

$$
\Theta_{x y}:=\left(\frac{|\mathfrak{m}|^{2} S}{1-|\mathfrak{m}|^{2} S}\right)_{x y}, \quad x, y \in \mathbb{T}_{L} .
$$

Note that the matrix $\Theta$ is the solution of

$$
\Theta=|\mathfrak{m}|^{2} S \Theta+|\mathfrak{m}|^{2} S
$$

which is obtained from (1.1) by dropping the error term $\mathcal{E}$. For a precise formulation of the result, we consider the weighted average

$$
T_{x y}:=\sum_{i} S_{x i}\left|G_{i y}\right|^{2} .
$$

Theorem 2.4 (Diffusion profile). Assume (2.12) and

$$
(W / L)^{2} \leqslant \eta \leqslant 1, \quad L \ll W^{1+\frac{2}{7}}
$$

Then

$$
\begin{aligned}
T_{x y}-\Theta_{x y} & \prec \frac{1}{L \eta}, \\
T_{x y} & \prec \Upsilon_{x y}, \\
\left|G_{x y}-\delta_{x y} \mathfrak{m}\right|^{2} & \prec \Upsilon_{x y},
\end{aligned}
$$

where we defined

$$
\Upsilon_{x y} \equiv \Upsilon_{x y}^{(K)}:=\frac{1}{L \eta}+\frac{1}{W \sqrt{\eta}} \exp \left[-\frac{\sqrt{\alpha \eta}}{W \sqrt{D}}|x-y|_{L}\right]+\frac{1}{W}\left\langle\frac{\sqrt{\eta}|x-y|_{L}}{W}\right\rangle^{-K}
$$

Here $K$ is an arbitrary, fixed, positive integer. All estimates are uniform in $z \in \mathbf{S}$ and $x, y \in \mathbb{T}_{L}$. 
Remark 2.5. Theorem 2.4 improves the analogous result in [9] where it was assumed that $L \ll W^{1+\frac{1}{4}}$. However, one expects that (2.20) and the local semicircle law (2.15) should in fact hold under the weaker conditions $\eta \gg 1 / L$ and $L \ll W^{2}$, from which one could deduce the complete delocalization of the eigenvectors for all $L \ll W^{2}$.

As explained in Section 2.2 in [9], $\Theta$ can be interpreted in terms of random walks. In fact, since $S$ is translation invariant, also $\Theta$ is so and it can be written as

$$
\Theta_{x y}=\sum_{n \geqslant 1}|\mathfrak{m}|^{2 n}\left(S^{n}\right)_{x y} \approx \sum_{n \geqslant 1} \mathrm{e}^{-n \alpha \eta}\left(S^{n}\right)_{x y},
$$

where we used (3.6) below and $\alpha$ is defined in (1.4). Moreover, $S$ is the transition matrix of a random walk on $\mathbb{T}_{L}$ whose steps are of size $W$, therefore $\Theta_{x y}$ is a superposition of random walks up to times of order $(\alpha \eta)^{-1}$.

The normalized variance of each step is given by the unrenormalized diffusion constant $D$ appearing in (1.2):

$$
D:=\frac{1}{2} \sum_{u}\left(\frac{u}{W}\right)^{2} S_{u 0}=D_{\infty}+O\left(W^{-1}\right) \quad \text { where } \quad D_{\infty}:=\frac{1}{2} \int x^{2} f(x) \mathrm{d} x .
$$

Proposition 2.8 in [9] provides an explicit formula for $\Theta$ : for each $K \in \mathbb{N}$ we have

$$
\Theta_{x y}=\theta_{x-y}+O\left(\frac{1}{W^{2}}\right)+O_{K}\left(\frac{1}{W}\left\langle\frac{\sqrt{\eta}|x-y|_{L}}{W}\right\rangle^{-K}\right)
$$

where

$$
\theta_{x}:=\frac{|\mathfrak{m}|^{2}}{L} \sum_{p \in \frac{2 \pi}{L} \mathbb{Z}} \mathrm{e}^{\mathrm{i} p x} \frac{1}{\alpha \eta+W^{2} D p^{2}}=\frac{|\mathfrak{m}|^{2}}{2 W \sqrt{D \alpha \eta}} \sum_{k \in \mathbb{Z}} \exp \left[-\frac{\sqrt{\alpha \eta}}{W \sqrt{D}}|x+k L|\right] .
$$

From (2.26), (2.27) and (2.23) we see that $\Theta_{x y} \leqslant C \Upsilon_{x y} \leqslant C^{\prime} \Phi^{2}$. The total mass of the profile $\sum_{x} \theta_{x}$ is simply given by

$$
\sum_{x} \theta_{x}=\frac{|\mathfrak{m}|^{2}}{\alpha \eta}=\frac{\operatorname{Im} \mathfrak{m}}{\eta}(1+O(\eta))
$$

where in the last step we used Lemma 3.5 below. Hence, the average height of the profile is of order $(L \eta)^{-1}$, while its maximum is of order $(W \sqrt{\eta})^{-1}$ : this means that when $\eta \gg(W / L)^{2}$ the profile is concentrated in the region $|x-y| \leqslant W \eta^{-1 / 2} \ll L$ and the complete delocalization has not taken place. For more details about the physical interpretation of $\Theta$ we refer to $[6,9]$.

\section{Preliminaries}

In this Section we work in the $d$-dimensional setting. The following lemma collects basic algebraic properties of stochastic domination $\prec$.

Lemma 3.1 (Basic properties of $\prec$ ).

(i) Suppose that $X(v) \prec Y(v)$ for all $v \in V$. If $|V| \leqslant N^{C}$ for some constant $C$ then $\sum_{v \in V} X(v) \prec \sum_{v \in V} Y(v)$.

(ii) Suppose that $X_{1} \prec Y_{1}$ and $X_{2} \prec Y_{2}$. Then $X_{1} X_{2} \prec Y_{1} Y_{2}$.

(iii) Suppose that $X \leqslant N^{C}$ and $Y \geqslant N^{-C}$ for some constant $C>0$. Then $X \prec Y$ implies $\mathbb{E}[X] \prec \mathbb{E}[Y]$.

If the above random variables depend on some parameter $u$ and the hypotheses are uniform in $u$ then so are the conclusions.

Proof. The proof follows from the definition of stochastic domination together with a union bound argument. 
Note that if for any $\varepsilon>0$ and $p \geqslant 1$ we have $\mathbb{E}|X|^{p} \leqslant N^{\varepsilon} \Psi^{p}$ for large enough $N$ which depends on $\varepsilon$ and $p$, then $X \prec \Psi$ by Chebyshev's inequality.

A crucial tool in our analysis is the cumulant expansion formula: the following version of the cumulant expansion formula is proved in [12]. Slightly different versions of the same formula can be found in $[4,13,14])$.

Lemma 3.2 (Cumulant expansion). Let $h$ be a complex random variable with all its moments exist. The $(p, q)$ cumulant of $h$ is defined as

$$
\mathcal{C}^{(p, q)}(h):=\left.(-i)^{p+q} \cdot\left(\frac{\partial^{p+q}}{\partial s^{p} \partial t^{q}} \log \mathbb{E} e^{\mathrm{i} s h+\mathrm{i} t \bar{h}}\right)\right|_{s=t=0}
$$

Let $f: \mathbb{C}^{2} \rightarrow \mathbb{C}$ be a smooth function, and we denote its holomorphic derivatives by

$$
f^{(p, q)}\left(z_{1}, z_{2}\right):=\frac{\partial^{p+q}}{\partial z_{1}^{p} \partial z_{2}^{q}} f\left(z_{1}, z_{2}\right) .
$$

Then for any fixed $\ell \in \mathbb{N}$, we have

$$
\mathbb{E} f(h, \bar{h}) \bar{h}=\sum_{p+q=0}^{\ell} \frac{1}{p ! q !} \mathcal{C}^{(p, q+1)}(h) \mathbb{E} f^{(p, q)}(h, \bar{h})+R_{\ell+1},
$$

given all integrals in (3.1) exists. Here $R_{\ell+1}$ is the remainder term depending on $f$ and $h$, and for any $\tau>0$, we have the estimate

$$
\begin{aligned}
R_{\ell+1}= & O(1) \cdot \mathbb{E}\left|h^{\ell+2} \cdot \mathbf{1}_{\left\{|h|>N^{\tau-1 / 2}\right\}}\right| \cdot \max _{p+q=\ell+1}\left\|f^{(p, q)}(z, \bar{z})\right\|_{\infty} \\
& +O(1) \cdot \mathbb{E}|h|^{\ell+2} \cdot \max _{p+q=\ell+1}\left\|f^{(p, q)}(z, \bar{z}) \cdot \mathbf{1}_{\left\{|z| \leqslant N^{\tau-1 / 2}\right\}}\right\|_{\infty} .
\end{aligned}
$$

The following result gives bounds on the cumulants of the entries of $H$.

Lemma 3.3. If $H$ satisfies (2.5) and (2.6) then for every $i, j \in \llbracket N \rrbracket$ and $k \in \mathbb{N}$ we have

$$
\mathcal{C}^{(p, q)}\left(H_{i j}\right)=O_{k}\left(S_{i j}^{k / 2}\right)=O_{k}\left(M^{-k / 2}\right), \quad p+q=k
$$

and $\mathcal{C}^{(0,1)}\left(H_{i j}\right)=\mathcal{C}^{(1,0)}\left(H_{i j}\right)=0$.

Proof. This follows easily by the homogeneity of the cumulants.

Moreover, we need the following bound on $\Lambda$, which was proved in [10] and [8] with two different approaches.

Lemma 3.4. We have

$$
\Lambda(z) \prec \frac{1}{\sqrt{M \eta}}
$$

uniformly for $z \in \mathbf{S}$.

Finally, we collect some elementary facts about $\mathfrak{m}$ which were proved in Lemma 3.5 in [9].

Lemma 3.5. (i) We have the identity

$$
1-|\mathfrak{m}|^{2}=\frac{\eta|\mathfrak{m}|^{2}}{\operatorname{Im} \mathfrak{m}}
$$

(ii) There is a constant $c>0$ such that for $z \in \mathbf{S}$

$$
c \leqslant|\mathfrak{m}| \leqslant 1 .
$$

(iii) For $z \in \mathbf{S}$ and $\alpha$ given in (1.4)

$$
|\mathfrak{m}|^{2}=1-\eta \alpha+O\left(\eta^{2}\right)
$$

(iv) For $z \in \mathbf{S}$

$$
\operatorname{Im} \mathfrak{m} \asymp 1, \quad 1-|\mathfrak{m}|^{2} \asymp \eta
$$

where the implicit constants in the two estimates depend on $\kappa$. 


\section{Self-consistent equation for $T$ and Fourier space analysis}

From this section up to Section 8 we will work in $d=1$, while in Section 9 we will discuss the high dimensions case. Following [9], we write a self-consistent equation for $T$ defined in (2.19) and we control its error term by using a Fourier space argument. Set

$$
\mathcal{E}_{x y}:=T_{x y}-|\mathfrak{m}|^{2} \sum_{i} S_{x i} T_{i y}-|\mathfrak{m}|^{2} S_{x y}
$$

so that $T$ satisfies (1.1). We introduce the projection $\Pi:=\mathbf{i i}^{*}$ with $\mathbf{i}=L^{-1 / 2}(1, \ldots, 1)$ and we denote the complementary projection by $\bar{\Pi}:=I-\Pi$ where $I$ is the identity matrix. Setting $\bar{T}_{y}=(\Pi T)_{x y}$, from Proposition 5.1 in [9] we know that

$$
T_{x y}=\bar{T}_{y}+|\mathfrak{m}|^{2}\left(\frac{S-\Pi}{I-|\mathfrak{m}|^{2} S}\right)_{x y}+\tilde{\mathcal{E}}_{x y}
$$

where

$$
\bar{T}_{y}=\frac{\operatorname{Im} \mathfrak{m}}{L \eta}\left[1+O_{\prec}\left(\frac{1}{\sqrt{W \eta}}\right)\right]
$$

and

$$
\tilde{\mathcal{E}}_{x y}=\left(\frac{1}{I-|\mathfrak{m}|^{2} S} \bar{\Pi} \mathcal{E}\right)_{x y} .
$$

Note that $\frac{\operatorname{Im} \mathfrak{m}}{L \eta}=\Pi_{x y} \frac{\operatorname{Im} \mathfrak{m}}{\eta}$ and

$$
\frac{\operatorname{Im} \mathfrak{m}}{\eta} \Pi+|\mathfrak{m}|^{2}\left(\frac{S-\Pi}{I-|\mathfrak{m}|^{2} S}\right)=\Theta
$$

where we used (3.4) and the fact that $\Pi S=S \Pi=\Pi$. Thus, from (4.2) and (4.3) we get

$$
T_{x y}=O_{\prec}\left(\frac{1}{L \sqrt{W \eta^{3}}}\right)+\Theta_{x y}+\tilde{\mathcal{E}}_{x y} .
$$

In order to get a bound for $\tilde{\mathcal{E}}$, we will analyze it in Fourier space. We introduce our conventions for the discrete Fourier transform: given $f: \mathbb{T}_{L} \rightarrow \mathbb{C}$ and $p \in \frac{2 \pi}{L} \mathbb{T}_{L}=: \mathbb{T}_{L}^{*}$, then we set

$$
\widehat{f}(p):=\sum_{x \in \mathbb{T}_{L}} \mathrm{e}^{-\mathrm{i} p x} f_{x}, \quad f_{x}=\frac{1}{L} \sum_{p \in \mathbb{T}_{L}^{*}} \mathrm{e}^{\mathrm{i} p x} \widehat{f}(p) .
$$

We define the family of vectors $\left(\mathbf{e}(p), p \in \mathbb{T}_{L}\right)$ whose components are $e_{x}(p):=L^{-1 / 2} \mathrm{e}^{\mathrm{i} p x}$. Thus, one can write $\widehat{f}(p)=L^{1 / 2}\langle\mathbf{e}(p), f\rangle$, where $\langle\cdot, \cdot\rangle$ is the standard inner product of $L^{2}\left(\mathbb{T}_{L}\right)$. Note that $\mathbf{e}(p)$ is completely delocalized for all $p \in \mathbb{T}_{L}^{*}$, in the sense that $\|\mathbf{e}(p)\|_{\infty} \leqslant L^{-1 / 2}$.

In the following proposition we get a bound for $\tilde{\mathcal{E}}_{x y}$ via a Fourier space argument. The idea is to split $\tilde{\mathcal{E}}$ in three parts corresponding to the zero mode, the low modes and the high modes contribution. The treatment of the first and the third term is very close to what is done in section 5 of [9], while the second term is analyzed by exploring the quadratic behaviour of the Fourier transform of $S_{x 0}$ for small momenta (see (1.2)).

Proposition 4.1. Let $\tilde{\mathcal{E}}_{x y}$ be defined as in (4.4). Then

$$
\sup _{x, y}\left|\tilde{\mathcal{E}}_{x y}\right| \prec \frac{1}{\sqrt{L}} \min \left(\frac{L^{2}}{W^{2}}, \frac{L}{W \sqrt{\eta}}\right) \sup _{y} \sup _{p \neq 0}\left|\langle\mathbf{e}(p), \mathcal{E}\rangle_{y}\right|+\sup _{x, y}\left|\mathcal{E}_{x y}\right|,
$$

where $\langle\mathbf{e}(p), \mathcal{E}\rangle_{y}=\sum_{x \in \mathbb{T}_{L}} e_{-x}(p) \mathcal{E}_{x y}$. Here $\mathcal{E}_{x y}$ is regarded as a vector in $x$, while the $y$ 's are just considered as parameters. 
Proof. We will use this trivial bound: let $\mathbf{w} \in \mathbb{C}^{L}$, then

$$
\|\mathbf{w}\|_{\infty} \leqslant\|\mathbf{w}\|_{2} \leqslant \sqrt{L}\|\mathbf{w}\|_{\infty} .
$$

Let $Q=\left(Q_{x y}, x, y \in \mathbb{T}_{L}\right)$ be a $L \times L$ matrix with translation invariant and $L$-periodic entries, i.e. there exists a function $q: \mathbb{T}_{L} \rightarrow \mathbb{C}$ such that $q_{x}=Q_{x 0}$. We now specify the form of $q_{x}$ via its discrete Fourier transform: for $p \in \mathbb{T}_{L}^{*}$ we set

$$
\widehat{q}(p)=1-\chi(p W)
$$

where $\chi \in C^{\infty}(\mathbb{R})$ is a bump function such that $\chi(r)=1$ for $|r|^{2} \leqslant 1$ and $\chi(r)=0$ for $|r|^{2} \geqslant 2$. Basically, $\widehat{q}(p)$ is a smoothed version of the indicator function $\mathbf{1}\left(p>W^{-1}\right)$. Furthermore, we introduce the notation $w_{x}:=(\bar{\Pi} \mathcal{E})_{x y}$, where we regard the index $y$ as a parameter.

Note that $Q$ and $S$ commute because they are translation invariant. Hence the error term (4.4) can be written as

$$
\tilde{\mathcal{E}}=\frac{I-Q}{I-|\mathfrak{m}|^{2} S} \mathbf{w}+\frac{Q}{I-|\mathfrak{m}|^{2} S} \mathbf{w} .
$$

From the definition of $\widehat{q}(p)$, one can easily see that the first term on the right hand side of (4.7) is the small Fourier modes contribution, while the second one is the large Fourier modes contribution. Let us analyze the large modes term: its $\ell^{\infty}$-norm can be bounded as follows

$$
\begin{aligned}
\left\|\frac{Q}{I-|\mathfrak{m}|^{2} S} \mathbf{w}\right\|_{\infty} & \leqslant \sum_{k=0}^{K-1}|\mathfrak{m}|^{2 k}\left\|Q S^{k} \mathbf{w}\right\|_{\infty}+\sum_{k=K}^{\infty}|\mathfrak{m}|^{2 k}\left\|Q S^{k} \mathbf{w}\right\|_{\infty} \\
& \leqslant \sum_{k=0}^{K-1}|\mathfrak{m}|^{2 k}\left\|Q S^{k}\right\|_{\infty \rightarrow \infty}\|\mathbf{w}\|_{\infty}+\sum_{k=K}^{\infty}|\mathfrak{m}|^{2 k}\left\|Q S^{k} \mathbf{w}\right\|_{2} \\
& \leqslant \sum_{k=0}^{K-1}|\mathfrak{m}|^{2 k}\left\|Q S^{k}\right\|_{\infty \rightarrow \infty}\|\mathbf{w}\|_{\infty}+\sqrt{L} \sum_{k=K}^{\infty}|\mathfrak{m}|^{2 k}\left\|Q S^{k}\right\|_{2 \rightarrow 2}\|\mathbf{w}\|_{\infty}
\end{aligned}
$$

where we used (4.6) and $K \in \mathbb{N}$ is going to be chosen later. Here we denoted the $\ell^{\infty} \rightarrow \ell^{\infty}$ norm of a matrix $A$ by $\|A\|_{\infty \rightarrow \infty}=\max _{i} \sum_{j}\left|A_{i j}\right|$ and the Euclidean matrix norm by $\|A\|_{2 \rightarrow 2}$.

We observe that

$$
\begin{aligned}
& \left\|S^{k} Q\right\|_{\infty \rightarrow \infty}=O(\log L) \\
& \left\|S Q^{1 / k}\right\|_{2 \rightarrow 2} \leqslant 1-c
\end{aligned}
$$

for some small positive $c$.

Note that $\|S\|_{\infty \rightarrow \infty} \leqslant 1$ since $\sup _{x}\left|\sum_{y} S_{x y} v_{y}\right| \leqslant \sup _{y}\left|v_{y}\right|=\|\mathbf{v}\|_{\infty}$ for any $\mathbf{v} \in \mathbb{C}^{L}$. Thus, to prove (4.8) we need to show that $\|Q\|_{\infty \rightarrow \infty}=\|q\|_{1}=O(\log L)$.

To do this, we consider $u(r)=1-\chi(r W)$ as smooth function on the torus, i.e. $r \in \tilde{\mathbb{T}}=[-\pi, \pi]$, with Fourier coefficients

$$
\widehat{u}(n):=\frac{1}{2 \pi} \int_{\widetilde{\mathbb{T}}} \mathrm{d} r \mathrm{e}^{\mathrm{i} r n} u(r), \quad n \in \mathbb{Z} .
$$

Since $u(r)$ is smooth, then for any $\ell \geqslant 0$ we have

$$
|\widehat{u}(n)| \leqslant C\left(\mathbf{1}(n=0)+\frac{\mathbf{1}(n \neq 0)}{W}\left|\frac{W}{n}\right|^{\ell}\right) .
$$

Note that $\widehat{q}(p)=u(p)$ for $p \in \mathbb{T}_{L}^{*}$, then we can write

$$
q_{x}=\frac{1}{L} \sum_{p \in \mathbb{T}_{L}^{*}} \mathrm{e}^{\mathrm{i} p x} \widehat{q}(p)=\frac{1}{L} \sum_{p \in \mathbb{T}_{L}^{*}} \mathrm{e}^{\mathrm{i} p x} u(p)=\sum_{m \in \mathbb{Z}} \widehat{u}(x+L m) .
$$


Using (4.10) and (4.11) and approximating the sums via integrals, one can see that

$$
\|q\|_{1} \leqslant \sum_{x \in \mathbb{T}_{L}} \sum_{m \in \mathbb{Z}}|\widehat{u}(x+L m)|=O(\log L),
$$

which shows (4.8).

On the other hand, to prove (4.9) we observe that a discrete Fourier analysis argument yields for some small positive $c$ that

$$
\mathbf{1}\left(|p|^{2} \geqslant W^{-2}\right)(1-\widehat{s}(p)) \geqslant c \mathbf{1}\left(|p|^{2} \geqslant W^{-2}\right),
$$

where $\widehat{s}$ is the Fourier transform of the function $s_{x}:=S_{x 0}$ for $x \in \mathbb{T}_{L}$. Hence, from (4.12) we get

$$
\left\|S Q^{1 / k}\right\|_{2 \rightarrow 2}=\sup _{p \in \mathbb{T}_{L}^{*}}\left|\widehat{s}(p)(1-\chi(p W))^{1 / k}\right| \leqslant \sup _{p \in \mathbb{T}_{L}^{*}}\left|\widehat{s}(p) \mathbf{1}\left(|p|^{2} \geqslant W^{-2}\right)\right| \leqslant 1-c .
$$

Thus, by (4.9) and (4.8) we obtain

$$
\left\|\frac{Q}{I-|\mathfrak{m}|^{2} S} \mathbf{w}\right\|_{\infty} \leqslant\|\mathbf{w}\|_{\infty}\left[O(K \log L)+\sqrt{L} \sum_{k=K}^{\infty}(1-c)^{k}\right] .
$$

By summing the geometric series and choosing $K=C \log L$ for some sufficiently large constant $C$ we get

$$
\left\|\frac{Q}{1-|\mathfrak{m}|^{2} S} \mathbf{w}\right\|_{\infty}=O(\log L)^{2}\|\mathbf{w}\|_{\infty} \prec\|\mathbf{w}\|_{\infty} .
$$

We now need to estimate the second term in (4.7), i.e. the small modes contribution, which can be written as

$$
\left(\frac{I-Q}{I-|\mathfrak{m}|^{2} S} \bar{\Pi} \mathcal{E}\right)_{x y}=\sum_{\substack{p \in \mathbb{T}_{L}^{*} \\ p \neq 0}} \frac{\chi(p W)}{1-|\mathfrak{m}|^{2} \widehat{s}(p)} e_{x}(p)\langle\mathbf{e}(p), \mathcal{E}\rangle_{y}
$$

where we recall that $\langle\mathbf{e}(p), \mathcal{E}\rangle_{y}=\sum_{x \in \mathbb{T}_{L}} e_{-x}(p) \mathcal{E}_{x y}$. Using (3.7) and the constraint on the momentum $p$ given by $\widehat{P}(p)$ and $1-\widehat{s}(p) \geqslant c W^{2} p^{2}$ for $(p W)^{2} \leqslant 2$, one can see that

$$
\left\|\sum_{\substack{p \in \mathbb{T}_{L}^{*} \\ p \neq 0}} \frac{\chi(p W)}{1-|\mathfrak{m}|^{2} \widehat{s}(p)} \mathbf{e}(p)\langle\mathbf{e}(p), \mathcal{E}\rangle_{y}\right\|_{\infty} \prec \frac{1}{\sqrt{L}} \sup _{p \neq 0}\left|\langle\mathbf{e}(p), \mathcal{E}\rangle_{y}\right| \sum_{\substack{p \in \mathbb{T}_{L}^{*} \\ p \neq 0}} \frac{\chi(p W)}{\eta+(p W)^{2}} .
$$

Note that

$$
\sum_{\substack{p \in \mathbb{T}_{L}^{*} \\ p \neq 0}} \frac{\chi(p W)}{\eta+W^{2}|p|^{2}} \prec \sum_{\substack{j \in \mathbb{Z} \\ 0<|j| \leqslant L / W}} \frac{1}{\eta+\left(\frac{W|j|}{L}\right)^{2}} \prec \frac{L^{2}}{W^{2}} \sum_{j=1}^{\infty}|j|^{-2} \prec \frac{L^{2}}{W^{2}}
$$

On the other hand, by estimating the sum with an integral we get

$$
\sum_{\substack{j \in \mathbb{Z} \\ 0<|j| \leqslant L / W}} \frac{1}{\eta+\left(\frac{W|j|}{L}\right)^{2}} \prec \int_{0}^{L / W} \frac{\mathrm{d} x}{\eta+\left(\frac{W x}{L}\right)^{2}} \prec \frac{L}{W \sqrt{\eta}} .
$$

Thus, by using (4.13), (4.14), (4.15) and (4.16) we obtain

$$
\sup _{x, y}\left|\tilde{\mathcal{E}}_{x y}\right| \prec \frac{1}{\sqrt{L}} \min \left(\frac{L^{2}}{W^{2}}, \frac{L}{W \sqrt{\eta}}\right) \sup _{y} \sup _{p \neq 0}\left|\langle\mathbf{e}(p), \mathcal{E}\rangle_{y}\right|+\sup _{x, y}\left|\mathcal{E}_{x y}\right| .
$$


Remark 4.2. For $T_{x y}^{\prime}=\sum_{i}\left|G_{x i}\right|^{2} S_{i y}$ an analogous result holds: it satisfies the self-consistent equation

$$
T^{\prime}=|\mathfrak{m}|^{2} T^{\prime} S+|\mathfrak{m}|^{2} S+\mathcal{E}^{\prime}
$$

so that one gets

$$
T^{\prime}=O_{\prec}\left(\frac{1}{L \sqrt{W \eta^{3}}}\right)+\Theta+\tilde{\mathcal{E}}^{\prime}, \quad \tilde{\mathcal{E}}^{\prime}=\mathcal{E}^{\prime} \bar{\Pi}\left(I-|\mathfrak{m}|^{2} S\right)^{-1} .
$$

In the proof of Proposition 4.1 we regarded $\mathcal{E}_{x y}$ as a column vector indexed by $x$ and we regarded $y$ as a parameter. Conversely, the analogous statement for $T^{\prime}$ can be obtained with the same Fourier analysis argument by seeing $\mathcal{E}_{x y}^{\prime}$ as a row vector indexed by $y$ with $x$ as parameter.

Remark 4.3. Note that in [9] the authors derive the bound

$$
\sup _{x, y}\left|\tilde{\mathcal{E}}_{x y}\right| \prec \min \left(\frac{L^{2}}{W^{2}}, \frac{L}{W \sqrt{\eta}}\right) \sup _{x, y}\left|\mathcal{E}_{x y}\right|
$$

and then use the fluctuation averaging estimates based on the Schur's complement formula to control $\sup _{x, y}\left|\mathcal{E}_{x y}\right|$. In (4.17) we see that in the first term, i.e. the small modes contribution, we gain a prefactor $L^{-1 / 2}$ compared to the prefactor in (4.18), but we have to estimate $\sup _{y} \sup _{p \neq 0}\left|\langle\mathbf{e}(p), \mathcal{E}\rangle_{y}\right|$ instead of just $\sup _{x, y}\left|\mathcal{E}_{x y}\right|$.

On the other hand, the second term in (4.17), corresponding to the large Fourier modes, is going to be always subdominant compared to the first one, since its prefactor is just 1.

In the following section we discuss how to estimate $\sup _{y} \sup _{p \neq 0}\left|\langle\mathbf{e}(p), \mathcal{E}\rangle_{y}\right|$ and $\sup _{x, y}\left|\mathcal{E}_{x y}\right|$ and we prove the main results. As already mentioned in the Introduction, for this task we will avoid using cumbersome expansions based on the Schur's complement formula, but we will rather employ the cumulant expansion method.

\section{Proofs of the main results}

Let us define the following family of vectors:

$$
\mathbb{V}:=\left\{\mathbf{v} \in \mathbb{C}^{L}:\|\mathbf{v}\|_{2}=O(1), \quad\|\mathbf{v}\|_{\infty}=O\left(L^{-1 / 2}\right)\right\} .
$$

Note that $\mathbf{e}(p) \in \mathbb{V}$ for any $p \in \mathbb{T}_{L}^{*}$. Moreover, let us fix the following notation: let $A$ be a $L \times L$ matrix and $\mathbf{u} \in \mathbb{C}^{L}$, then we will write $A_{\mathbf{u} b}:=\sum_{a} u_{a} A_{a b}$. Thus

$$
\sup _{y} \sup _{p \neq 0}\left|\langle\mathbf{e}(p), \mathcal{E}\rangle_{y}\right| \leqslant \sup _{y} \sup _{\mathbf{v} \in \mathbb{V}}\left|\mathcal{E}_{\mathbf{v} y}\right| .
$$

Furthermore, it is convenient to split $\mathcal{E}_{x y}$ as $\mathcal{E}_{x y}=\mathcal{P}_{x y}+\mathcal{R}_{x y}$ where

$$
\begin{aligned}
& \mathcal{P}_{x y}=-\mathfrak{m} z T_{x y}-\mathfrak{m} \sum_{i, j} S_{x i} S_{i j} G_{j j}\left|G_{i y}\right|^{2}-\mathfrak{m} \sum_{i, j} S_{x i} S_{i j} \bar{G}_{i i}\left|G_{j y}\right|^{2}-\mathfrak{m} S_{x y} \bar{G}_{y y} \\
& \mathcal{R}_{x y}=\mathfrak{m} \sum_{i, j} S_{x i} S_{i j}\left(G_{j j}-\mathfrak{m}\right)\left|G_{i y}\right|^{2}+\mathfrak{m} \sum_{i, j} S_{x i} S_{i j}\left(\bar{G}_{i i}-\overline{\mathfrak{m}}\right)\left|G_{j y}\right|^{2}+\mathfrak{m} S_{x y}\left(\bar{G}_{y y}-\overline{\mathfrak{m}}\right) .
\end{aligned}
$$

By using (2.10) in (5.2) we readily see that indeed

$$
\mathcal{P}_{x y}=\left(1+\mathfrak{m}^{2}\right) T_{x y}-\mathfrak{m} \sum_{i, j} S_{x i} S_{i j} G_{j j}\left|G_{i y}\right|^{2}-\mathfrak{m} \sum_{i, j} S_{x i} S_{i j} \bar{G}_{i i}\left|G_{j y}\right|^{2}-\mathfrak{m} S_{x y} \bar{G}_{y y}=\mathcal{E}_{x y}-\mathcal{R}_{x y} .
$$

The reason why we chose to write $\mathcal{E}_{x y}$ in this complicated way is that, applying the cumulant expansion directly to $\mathbb{E} \mathcal{E}_{x y}$, it is not possible to get any cancellation even at the level of the expectation, while for $\mathcal{P}_{x y}$ one gets $\mathbb{E} \mathcal{P}_{x y}=0$ when $H_{i j}$ are Gaussian. 
To see that, we recall the basic definition of the Green function $G(z)=(H-z)^{-1}$ which amounts to

$$
z G=H G-z I .
$$

We set the notation $\partial_{i j} g(H):=\frac{\partial}{\partial H_{i j}} g(H)$ and we recall also the differentiation rule when $H$ is complex Hermitian:

$$
\partial_{k l} G_{i j}=-G_{i k} G_{l j} .
$$

Note that, assuming $H_{i j}$ to be Gaussian with $\mathbb{E} H_{i j}^{2}=0$, in the cumulant expansion formula (3.1) only the term dependent on the variance $\mathbb{E}\left|H_{i j}\right|^{2}$ survives:

$$
\mathbb{E} H_{i j} f\left(H_{i j}, H_{j i}\right)=\mathbb{E}\left|H_{i j}\right|^{2} \mathbb{E} \partial_{j i} f\left(H_{i j}, H_{j i}\right)=S_{i j} \mathbb{E} \partial_{j i} f\left(H_{i j}, H_{j i}\right) .
$$

Thus, from (5.4), (5.5) and (5.6), we easily get

$$
\begin{aligned}
z \mathbb{E} T_{x y}+S_{x y} \mathbb{E} \bar{G}_{y y} & =\sum_{i} S_{x i}\left(z G_{i y}\right) \bar{G}_{i y}=\mathbb{E} \sum_{i, j} S_{x i} H_{i j} G_{j y} \bar{G}_{i y}=\mathbb{E} \sum_{i, j} S_{x i} S_{i j} \partial_{j i}\left(G_{j y} \bar{G}_{i y}\right) \\
& =-\mathbb{E} \sum_{i, j} S_{x i} S_{i j}\left(G_{j j}\left|G_{i y}\right|^{2}+\bar{G}_{i i}\left|G_{j y}\right|^{2}\right)
\end{aligned}
$$

which implies that $\mathbb{E} \mathcal{P}_{x y}=0$. As we will see, remarkable cancellations occur also for the moments of $\mathcal{P}_{x y}$ even when $H$ is non Gaussian and $\mathbb{E} H_{i j}^{2} \neq 0$, allowing us to control $\mathcal{P}_{x y}$ and $\mathcal{P}_{\mathbf{v} y}$.

In the following, we shall call control parameter any positive and deterministic quantity $\Psi^{(N)}(z)$ and we shall call admissible any control parameter $\Psi^{(N)}(z)$ such that

$$
M^{-1 / 2} \leqslant \Psi^{(N)}(z) \leqslant M^{-\gamma / 2}
$$

for all $N$ and $z \in \mathbf{S}$, where $\gamma$ is the same fixed number as in (2.8). A typical example of an admissible control parameter is $\Psi(z)=\frac{1}{\sqrt{M \eta}}$.

The proposition below states precisely the bounds that we obtain for $\mathcal{P}_{x y}$ and $\mathcal{P}_{\mathbf{v} y}$, as well as for $\mathcal{R}_{\mathbf{v} y}$ and $\mathcal{R}_{x y}$ : it is the technical core of the paper and it replaces the fluctuation averaging estimates of Proposition 3.9 in [9].

Proposition 5.1. Let $\Psi$ be an admissible control parameter as defined in (5.7) such that $\Lambda \prec \Psi$ and $\mathbf{v} \in \mathbb{V}$. Then the following bounds hold true:

$$
\begin{aligned}
\mathcal{P}_{\mathbf{v} y} & \prec \frac{\Psi^{3}}{\sqrt{\eta}}+\frac{\Psi}{\sqrt{L \eta}}, \\
\mathcal{P}_{x y} & \prec \frac{\Psi^{3}}{W} \sqrt{\frac{L}{\eta}}+\frac{\Psi}{W \sqrt{\eta}}, \\
\mathcal{R}_{\mathbf{v} x} & \prec \frac{\Psi^{2}}{\eta \sqrt{L}}+\frac{\Psi}{\sqrt{L}}, \\
\mathcal{R}_{x y} & \prec \frac{\Psi^{2}}{W \eta}+\frac{\Psi}{W} .
\end{aligned}
$$

Proof. See Section 6.

To prove the main results we will need also a couple of auxiliary lemmata which show how to combine apriori bounds on $\Lambda$ and $T$ to get a better estimate for $\Lambda$. Note that the first one is basically Lemma 5.3 in [9] and in the Appendix we give a new proof which does not rely on the averaging fluctuation estimates of [7].

Lemma 5.2. Suppose that $\Lambda \prec \Psi$ for some admissible parameter $\Psi$ and $T_{a b}, T_{a b}^{\prime} \prec \Omega_{a b}^{2}$ for a family of admissible control parameters $\Omega_{a b}$ indexed by a pair $(a, b)$. Then

$$
\left|G_{a b}-\mathfrak{m} \delta_{a b}\right|^{2} \prec \Omega_{a b}^{2}+\Psi^{4} .
$$


Proof. See Appendix.

The second lemma implements the idea of self improving bounds: we start with a rough bound and we improve it by a recursive procedure.

Lemma 5.3. Suppose that

$$
\Lambda \prec \Psi, \quad \Omega \leqslant \Psi \leqslant \tilde{\Psi} ; \quad T_{i j}, T_{i j}^{\prime} \prec \Omega^{2}+\sum_{k=1}^{K} a_{k} \Psi^{k}
$$

where $\Psi, \tilde{\Psi}, \Omega$ are admissible control parameters and $K$ is some fixed integer. Assume also that $a_{k} \geqslant 0$ for $k=1, \ldots, K$ and

$$
a_{1} \Omega^{-1} \ll 1 ; \quad a_{\ell} \Psi^{\ell-2} \ll 1, \quad 2 \leqslant \ell \leqslant K
$$

Then $\Lambda \prec \Omega$.

Proof. Set $\Xi^{2}=\Omega^{2}+\sum_{k=1}^{K} a_{k} \Psi^{k}$. From Lemma 5.2, we easily deduce the implication

$$
\Lambda \prec \Psi \quad \Longrightarrow \quad \sup _{a, b}\left|G_{a b}-\mathfrak{m} \delta_{a b}\right|=\Lambda \prec \Xi+\Psi^{2} .
$$

After $k$ iterations of (5.14) we get $\Lambda \prec \Xi+\Psi^{2^{k}}$. Since $\Xi$ and $\Psi$ are both admissible control parameters, taking $k \sim|\log \gamma|$ we get the implication

$$
\Lambda^{2} \prec \Psi^{2} \quad \Longrightarrow \quad \Lambda^{2} \prec \Xi^{2}=\Omega^{2}+\sum_{k=1}^{K} a_{k} \Psi^{k} .
$$

We can iterate (5.15) by defining the recursion relation

$$
\Psi_{i+1}^{2}:=\Omega^{2}+\sum_{k=1}^{K} a_{k} \Psi_{i}^{k}, \quad \Psi_{0}=\tilde{\Psi} .
$$

Thus, (5.15) implies that $\Lambda^{2} \prec \Psi_{i}^{2}$ for any fixed $i$. The conditions (5.13) and the fact that $\Omega$ is admissible imply that there is a finite integer $i$ (depending on the implicit constants involved in the relation "«"), such that $\Psi_{i}^{2} \prec \Omega^{2}$.

We are now ready to prove our main results.

5.1. Proof of Theorem 2.2. From (5.1) and propositions 4.1 and 5.1 we get

$$
\begin{aligned}
\sup _{x, y}\left|\tilde{\mathcal{E}}_{x y}\right| & \left.\prec \frac{L^{3 / 2}}{W^{2}} \sup _{y} \sup _{\mathbf{v} \in \mathbb{V}}\left(\left|\mathcal{P}_{\mathbf{v} y}\right|+\left|\mathcal{R}_{\mathbf{v} y}\right|\right)+\sup _{x, y}\left|\mathcal{P}_{x y}\right|+\sup _{x, y}\left|\mathcal{R}_{x y}\right|\right) \\
& \prec \frac{L^{2}}{W^{2}}\left(\frac{\Psi^{2}}{L \eta}+\frac{\Psi^{3}}{\sqrt{L \eta}}+\frac{\Psi}{L \sqrt{\eta}}\right)+\frac{\Psi^{2}}{W \eta}+\frac{\Psi^{3}}{W} \sqrt{\frac{L}{\eta}}+\frac{\Psi}{W \sqrt{\eta}} .
\end{aligned}
$$

By (4.5), (2.26) and (5.16) we have

$$
T_{x y} \prec \Phi^{2}+\frac{L^{2}}{W^{2}}\left(\frac{\Psi^{2}}{L \eta}+\frac{\Psi^{3}}{\sqrt{L \eta}}+\frac{\Psi}{L \sqrt{\eta}}\right)+\frac{\Psi^{2}}{W \eta}+\frac{\Psi^{3}}{W} \sqrt{\frac{L}{\eta}}+\frac{\Psi}{W \sqrt{\eta}} .
$$

To finish the proof we apply Lemma 5.3 with $\Omega=\Phi$ and, thanks to Lemma $3.4, \tilde{\Psi}=(W \eta)^{-1 / 2}$. In this setting we have $\Psi^{-2} \leqslant L \eta+W \sqrt{\eta}$. The conditions (2.14) in Theorem 2.2 arise from the assumption (5.13) in Lemma 5.3. 
5.2. Proof of Theorem 2.4. We are now in the range $(W / L)^{2} \leqslant \eta \leqslant 1$, therefore (4.5), Proposition 4.1 and 5.1 imply that for some admissible control parameter $\Psi$ such that $\Lambda \prec \Psi$

$$
T_{x y}-\Theta_{x y} \prec \frac{L}{W \sqrt{\eta}}\left(\frac{\Psi^{2}}{L \eta}+\frac{\Psi^{3}}{\sqrt{L \eta}}+\frac{\Psi}{L \sqrt{\eta}}\right)+\frac{\Psi^{2}}{W \eta}+\frac{\Psi^{3}}{W} \sqrt{\frac{L}{\eta}}+\frac{\Psi}{W \sqrt{\eta}} .
$$

From Theorem 2.2 we see that, in the range $(W / L)^{2} \leqslant \eta \leqslant 1$, we have $\Psi=W^{-1 / 2} \eta^{-1 / 4}$ when

$$
\frac{W^{2}}{L^{2}} \gg \frac{L^{3 / 2}}{W^{5 / 2}} \Longleftrightarrow L \ll W^{9 / 7} .
$$

It is now easy to check from (5.17) with $\Psi=W^{-1 / 2} \eta^{-1 / 4},(W / L)^{2} \leqslant \eta \leqslant 1$ and $L \ll W^{9 / 7}$ that (2.20) holds true. Note that (2.21) follows by using (2.23) in (2.20). Finally, using Lemma 5.2 with $\Omega_{i j}^{2}=\Upsilon_{i j}$ and $\Psi=W^{-1 / 2} \eta^{-1 / 4}$, we obtain

$$
\left|G_{i j}-\delta_{i j} \mathfrak{m}\right|^{2} \prec \Upsilon_{i j}+\Psi^{4} \prec \Upsilon_{i j}
$$

Here we used that $\Psi^{4}$ can be absorbed into $(L \eta)^{-1} \leqslant \Upsilon_{i j}$. This proves (2.22), and hence concludes the proof of Theorem 2.4.

5.3. Proof of Corollary 2.3. From $(2.17)$ we see that we need $\Lambda^{2} \prec(L \eta)^{-1}$. From Theorem 2.2 we know that this is true when $\eta \leqslant(W / L)^{2}$ and $\eta \gg L^{3 / 2} W^{-5 / 2}$. Therefore, we have to require that

$$
\frac{L^{3 / 2}}{W^{5 / 2}} \ll \frac{W^{2}}{L^{2}}
$$

which is true when $L \ll W^{9 / 7}$.

\section{Proof of Proposition 5.1}

In order to avoid useless technical complications we assume that $H$ is Gaussian and Hermitian and that

$$
\mathbb{E} \zeta_{i j}^{2}=0 \text { for all } i<j .
$$

in addition to (2.4). For example, (6.1) is true when the real and imaginary parts of $\zeta_{i j}$ are independent with identical variance. However, our results hold also without these assumptions and in Section 8 we sketch how to achieve this generalization.

Let us define a family of matrices $\mathbb{S}$ such that

$$
\mathbb{S}=\left\{\sigma \in \mathbb{C}^{L \times L}: \sup _{x, y}\left|\sigma_{x y}\right| \prec W^{-1}, \quad \sum_{x}\left|\sigma_{x y}\right| \prec 1, \quad \sum_{y}\left|\sigma_{x y}\right| \prec 1\right\} .
$$

Note that $\mathbb{S}$ is closed under matrix addition and multiplication. In particular, we immediately see that $S \in \mathbb{S}$. The following lemma, proven in Section 6.1, collects all the necessary estimates needed to prove Proposition 5.1.

Lemma 6.1. Let be $\Psi$ an admissible control parameter defined as in (5.7) and such that $\Lambda \prec \Psi$.

(i) Let $\sigma^{(1)}, \sigma^{(2)}, \ldots, \sigma^{(n)} \in \mathbb{S}$, then for $n \geqslant 1$ we have

$$
Y_{a b ; u_{1} \cdots u_{n}}^{(n)}:=\sum_{i_{1}, \ldots, i_{n}} \sigma_{u_{1} i_{1}}^{(1)} \cdots \sigma_{u_{n} i_{n}}^{(n)} G_{a i_{1}} G_{i_{1} i_{2}} \cdots G_{i_{n-1} i_{n}} G_{i_{n} b} \prec \Psi^{2 n+1}+\delta_{a b} \Psi^{2 n} .
$$

(ii) Let $\sigma^{(1)}, \sigma^{(2)}, \ldots, \sigma^{(n)} \in \mathbb{S}$, then for $n \geqslant 2$ and for some $\xi \in \mathbb{S}$ with nonnegative entries we have

$$
Z_{a b ; u_{3} \cdots u_{n}}^{(n)}:=\sum_{i_{1}, \ldots, i_{n}} \sigma_{a i_{1}}^{(1)} \sigma_{b i_{2}}^{(2)} \sigma_{u_{3} i_{3}}^{(3)} \cdots \sigma_{u_{n} i_{n}}^{(n)} G_{i_{1} i_{2}} \cdots G_{i_{n-1} i_{n}} G_{i_{n} i_{1}} \prec \Psi^{2 n}+\xi_{a b} \Psi^{2(n-2)} .
$$


(iii) Let $\sigma \in \mathbb{S}$, then we have

$$
X_{i}:=\sum_{j} \sigma_{i j}\left(G_{j j}-\mathfrak{m}\right) \prec \Psi^{2}, \quad X_{i j}:=\sum_{k} \sigma_{i k} G_{k j} \prec \Psi^{2} .
$$

Remark 6.2. In what follows we will refer to $Y_{a b ; u_{1} \ldots u_{n}}^{(n)}$ and $Z_{a b ; u_{3} \ldots u_{n}}^{(n)}$ respectively as open chain (or simply chain) and loop of order $n$. This terminology emphasizes that in $Y^{(n)}$ the extreme indices $a$ and $b$ of the product $G_{a i_{1}} G_{i_{1} i_{2}} \cdots G_{i_{n-1} i_{n}} G_{i_{n} b}$ are not summed over, while in $Z^{(n)}$ the extreme indices are identical and they are summed over. The order $n$ refers to the fact that both $Y^{(n)}$ and $Z^{(n)}$ involve $n$ summations.

The following lemma translates the control of arbitrary moment of a random variable into stochastic domination bounds.

Lemma 6.3. Let $\phi$ be a random variable such that $0 \leqslant \phi \leqslant L^{C}$ for some $C>0$ and let $\varphi \in \mathbb{R}^{+}$be deterministic such that $\varphi \in\left[L^{-C}, L^{C}\right]$. Suppose that there exists $q \in[0,1)$ such that for any deterministic $\vartheta \in\left[\varphi, L^{C}\right]$ and any $p \in \mathbb{N}$ one has the implication

$$
\phi \prec \vartheta \quad \Longrightarrow \quad \mathbb{E}|\phi|^{2 p}=\sum_{k=1}^{2 p} O_{\prec}\left(\left(\vartheta^{q} \varphi^{1-q}\right)^{k}\right) \mathbb{E}|\phi|^{2 p-k},
$$

then $\phi \prec \varphi$.

Proof. Applying Hölder inequality to (6.6) one gets

$$
\mathbb{E}|\phi|^{2 p} \leqslant \sum_{k=1}^{2 p} O_{\prec}\left(\left(\vartheta^{q} \varphi^{1-q}\right)^{k}\right)\left(\mathbb{E}|\phi|^{2 p}\right)^{\frac{2 p-k}{2 p}}
$$

which implies that

$$
\mathbb{E}|\phi|^{2 p}=O_{\prec}\left(\left(\vartheta^{q} \varphi^{1-q}\right)^{2 p}\right) .
$$

Then from Markov inequality we deduce the implication

$$
\phi \prec \vartheta \Longrightarrow \phi \prec \vartheta^{p} \varphi^{1-p} .
$$

By invoking Lemma 2.6 in [12], we conclude the proof.

We are now ready to prove Proposition 5.1. We will first prove (5.8).

Step 1. We recall that by spectral decomposition of $G$ we can easily get the so called Ward identity

$$
\sum_{i}\left|G_{x i}\right|^{2}=\frac{\operatorname{Im} G_{x x}}{\eta}
$$

Let us now define

$$
\mathcal{Q}_{x y}=\sum_{i, j} S_{\mathbf{v} i}\left(H_{i j} G_{j x} \bar{G}_{i y}+S_{i j} G_{j j} G_{i x} \bar{G}_{i y}+S_{i j} \bar{G}_{i i} G_{j x} \bar{G}_{j y}\right)
$$

and accordingly,

$$
\overline{\mathcal{Q}}_{x y}=\sum_{i, j} \bar{S}_{\mathbf{v} j}\left(H_{i j} G_{j y} \bar{G}_{i x}+S_{j i} G_{j j} \bar{G}_{i x} G_{i y}+S_{j i} \bar{G}_{i i} \bar{G}_{j x} G_{j y}\right),
$$

where we recall that $S_{\mathbf{v} i}=\sum_{x} v_{x} S_{x i}$ and $\|\mathbf{v}\|_{\infty}=O\left(L^{-1 / 2}\right)$. From (5.2) we see that $\mathcal{P}_{\mathbf{v} y}=-\mathfrak{m} \mathcal{Q}_{y y}$, and by (5.5) we have the derivatives

$$
\partial_{j i} \mathcal{Q}_{y y}=-\mathcal{Q}_{j y} G_{i y}-\mathcal{Q}_{y i} \bar{G}_{j y}+S_{\mathbf{v} j} G_{i y} \bar{G}_{j y}-\sum_{k, l} S_{\mathbf{v} k} S_{k l} G_{l j} G_{i l}\left|G_{k y}\right|^{2}-\sum_{k, l} S_{\mathbf{v} k} S_{k l} \bar{G}_{k i} \bar{G}_{j k}\left|G_{l y}\right|^{2}
$$


and

$$
\partial_{j i} \overline{\mathcal{Q}}_{y y}=-\overline{\mathcal{Q}}_{i y} \bar{G}_{j y}-\overline{\mathcal{Q}}_{y j} G_{i y}+\bar{S}_{\mathbf{v} j} G_{i y} \bar{G}_{j y}-\sum_{k, l} \bar{S}_{\mathbf{v} l} S_{l k} G_{l j} G_{i l}\left|G_{k y}\right|^{2}-\sum_{k, l} \bar{S}_{\mathbf{v} l} S_{l k} \bar{G}_{k i} \bar{G}_{j k}\left|G_{l y}\right|^{2}
$$

Now we fix $p \geqslant 2$, and by cumulant formula (5.6) we have

$$
\begin{aligned}
\mathbb{E}\left|\mathcal{Q}_{y y}\right|^{2 p} & =\mathbb{E} \sum_{i_{1}, j_{1}} S_{\mathbf{v} i_{1}}\left(H_{i_{1} j_{1}} G_{j_{1} y} \bar{G}_{i_{1} y}+S_{i_{1} j_{1}} G_{j_{1} j_{1}}\left|G_{i_{1} y}\right|^{2}+S_{i_{1} j_{1}} \bar{G}_{i_{1} i_{1}}\left|G_{j_{1} y}\right|^{2}\right) \cdot \mathcal{Q}_{y y}^{p-1} \overline{\mathcal{Q}}_{y y}^{p} \\
& =\mathbb{E} \sum_{i_{1}, j_{1}} S_{\mathbf{v} i_{1}} S_{i_{1} j_{1}} G_{j_{1} y} \bar{G}_{i_{1} y}\left[(p-1)\left(\partial_{j_{1} i_{1}} \mathcal{Q}_{y y}\right) \mathcal{Q}_{y y}^{p-2} \overline{\mathcal{Q}}_{y y}^{p}+p\left(\partial_{j_{1} i_{1}} \overline{\mathcal{Q}}_{y y}\right)\left|\mathcal{Q}_{y y}\right|^{2 p-2}\right] .
\end{aligned}
$$

Now we would like to compute the second line of (6.9) by recursively applying cumulant formula. To this end, we define for each $m \in\{2,3, \ldots, 2 p-1\}$ the set

$$
\mathcal{V}_{m-1, m}=\left\{G_{j_{m} j_{m-1}} G_{i_{m-1} y} \bar{G}_{i_{m} y}, \bar{G}_{i_{m} i_{m-1}} \bar{G}_{j_{m-1} y} G_{j_{m} y}\right\}
$$

For $n \in\{1,2, \ldots, 2 p-1\}$, let us consider

$$
\left(\frac{1}{\sqrt{L}}\right)^{n} \mathbb{E} \sum_{i_{1}, j_{1}, \ldots, i_{n}, j_{n}} \sigma_{i_{1} j_{1}}^{(1)} \cdots \sigma_{i_{n} j_{n}}^{(n)} G_{j_{1} y} \bar{G}_{i_{1} y} V_{1,2} \cdots V_{n-1, n}\left(\partial_{j_{n} i_{n}} \mathcal{Q}_{y y}\right) \mathcal{Q}_{y y}^{\alpha} \overline{\mathcal{Q}}_{y y}^{\beta}
$$

where $V_{m-1, m} \in \mathcal{V}_{m-1, m}$ for $m=2,3, \ldots, n, \sigma^{(1)}, \ldots, \sigma^{(n)} \in \mathbb{S}$, and $\alpha+\beta=2 p-n-1$. Formula (6.11) is one of the terms produced by applying $n$ times the cumulant expansion to $\mathbb{E}\left|\mathcal{Q}_{y y}\right|^{2 p}$ : for instance, if for $n=1$ we set $\sigma^{(1)}:=\sqrt{L} S_{\mathbf{v} i_{1}} S_{i_{1} j_{1}},(6.11)$ corresponds to the first term on the second line of (6.9). By the differential rule (6.8) we see that $(6.11)$ becomes

$$
\begin{aligned}
& \left(\frac{1}{\sqrt{L}}\right)^{n} \mathbb{E} \sum_{i_{1}, j_{1}, \ldots, i_{n}, j_{n}} \sigma_{i_{1} j_{1}}^{(1)} \cdots \sigma_{i_{n} j_{n}}^{(n)} G_{j_{1} y} \bar{G}_{i_{1} y} V_{1,2} \cdots V_{n-1, n} \cdot \mathcal{Q}_{y y}^{\alpha} \overline{\mathcal{Q}}_{y y}^{\beta} \cdot \\
& \left(-\mathcal{Q}_{j_{n} y} G_{i_{n} y}-\mathcal{Q}_{y i_{n}} \bar{G}_{j_{n} y}+S_{\mathbf{v} j_{n}} G_{i_{n} y} \bar{G}_{j_{n} y}-\sum_{i, j} S_{\mathbf{v} i} S_{i j} G_{j j_{n}} G_{i_{n} j}\left|G_{i y}\right|^{2}-\sum_{i, j} S_{\mathbf{v} i} S_{i j} \bar{G}_{i i_{n}} \bar{G}_{j_{n} i}\left|G_{j y}\right|^{2}\right) \\
& =:(A)+(B)+(C)+(D)+(E) .
\end{aligned}
$$

In the remaining proof we look at each term on the above carefully.

Step 2. Let us first look at term (C), which is

$$
\begin{aligned}
& \left(\frac{1}{\sqrt{L}}\right)^{n} \mathbb{E} \sum_{i_{1}, j_{1}, \ldots, i_{n}, j_{n}} \sigma_{i_{1} j_{1}}^{(1)} \cdots \sigma_{i_{n} j_{n}}^{(n)} G_{j_{1} y} \bar{G}_{i_{1} y} V_{1,2} \cdots V_{n-1, n} S_{\mathbf{v} j_{n}} G_{i_{n} y} \bar{G}_{j_{n} y} \cdot \mathcal{Q}_{y y}^{\alpha} \overline{\mathcal{Q}}_{y y}^{\beta} \\
= & \left(\frac{1}{\sqrt{L}}\right)^{n+1} \mathbb{E} \sum_{i_{1}, j_{1}, \ldots, i_{n}, j_{n}} \sigma_{i_{1} j_{1}}^{(1)} \cdots \sigma_{i_{n-1} j_{n-1}}^{(n-1)} \sigma_{i_{n} j_{n}}^{(n)} G_{j_{1} y} \bar{G}_{i_{1} y} V_{1,2} \cdots V_{n-1, n} G_{i_{n} y} \bar{G}_{j_{n} y} \cdot \mathcal{Q}_{y y}^{\alpha} \overline{\mathcal{Q}}_{y y}^{\beta},
\end{aligned}
$$

where in the second line we renamed $\sqrt{L} S_{\mathbf{v} j_{n}} \sigma_{i_{n} j_{n}}^{(n)} \in \mathbb{S}$ by $\sigma_{i_{n} j_{n}}^{(n)}$. By our definition of $\mathcal{V}$ we see that for $m=$ $1,2, \ldots, n-2, V_{m, m+1}$ contains either $\bar{G}_{i_{m+1} i_{m}}$ or $G_{j_{m+1} j_{m}}$. W.L.O.G. assume $G_{j_{2} j_{1}}$ is a factor of $V_{1,2}$, and let $k_{1} \in\{1,2,3, \ldots, n-2\}$ be the smallest integer such that $\bar{G}_{i_{k_{1}+2} i_{k_{1}+1}}$ is a factor of $V_{k_{1}+2, k_{1}+1}$. Thus

$$
V_{k_{1}+1, k_{1}}=G_{j_{k_{1}+1} j_{k_{1}}} G_{i_{k_{1}} y} \bar{G}_{i_{k_{1}+1} y}
$$

and $(\mathrm{C})$ contains

$$
G_{j_{k_{1}+1} j_{k_{1}}} \cdots G_{j_{2} j_{1}} G_{j_{1} y}
$$

This means that (C) actually contains a chain (in the sense of Remark 6.2 ) of order $k_{1}$. Now let $k_{2} \in\left\{k_{1}+1, \ldots, n-2\right\}$ be the smallest integer bigger than $k_{1}$ such that $G_{j_{k_{2}+2} j_{k_{2}+1}}$ is a factor of $V_{k_{2}+1, k_{2}+2}$, then

$$
V_{k_{2}+1, k_{2}}=\bar{G}_{i_{k_{2}+1} i_{k_{2}}} G_{j_{k_{2}} y} \bar{G}_{j_{k_{2}+1} y}
$$


and (6.14) shows that $(\mathrm{C})$ contains

$$
\bar{G}_{i_{k_{2}+1} i_{k_{2}}} \cdots \bar{G}_{i_{k_{1}+2} i_{k_{1}+1}} \bar{G}_{i_{k_{1}+1} y} .
$$

and consequently it contains another chain of order $k_{2}-k_{1}$. By continuing this process, we can find in (A) a product of finitely many different chains. Let $\mathcal{T}$ be the collection of all such $G$ and $\bar{G}$ that appear in all these chains, and for $m=1,2, \ldots, n-1$, let $q_{m} \in\left\{i_{m}, j_{m}\right\}$ denote the index that appears in one of the chains. Let $\left\{p_{m}\right\}:=\left\{i_{m}, j_{m}\right\} /\left\{q_{m}\right\}$ for $m \in\{1,2, \ldots, n-1\}$.

By Lemma 6.1 (i), we see that

$$
\sum_{j_{1}, \ldots, j_{k_{1}}} \sigma_{i_{1} j_{1}}^{(1)} \cdots \sigma_{i_{k_{1}} j_{k_{1}}}^{\left(k_{1}\right)} G_{j_{k_{1}+1} j_{k_{1}}} \cdots G_{j_{2} j_{1}} G_{j_{1} y} \prec \Psi^{2 k_{1}+1}+\Psi^{2 k_{1}} \delta_{j_{k_{1}+1} y} .
$$

Assume there are totally $l$ many chains. By applying the above estimate for other chains, we see that

$$
\sum_{q_{1}, \ldots, q_{n-1}} \prod_{m=1}^{n-1} \sigma_{i_{m} j_{m}}^{(m)} \prod_{t \in \mathcal{T}} t \prec\left(\Psi^{2 k_{1}+1}+\Psi^{2 k_{1}} \delta_{p_{k_{1}+1} y}\right)\left(\Psi^{2\left(k_{2}-k_{1}\right)+1}+\Psi^{2\left(k_{2}-k_{1}\right)} \delta_{p_{k_{2}+1} y}\right) \cdots\left(\Psi^{2\left(k_{l}-k_{l-1}\right)+1}+\Psi^{2\left(k_{l}-k_{l-1}\right)} \delta_{p_{n} y}\right),
$$

Note that the LHS of (6.15) is contained in (C). Together with (6.13) we have

$$
\begin{aligned}
(C) \prec & \left(\frac{1}{\sqrt{L}}\right)^{n+1}\left(\Psi^{2 k_{1}+1}+\Psi^{2 k_{1}} \delta_{p_{k_{1}+1} y}\right)\left(\Psi^{2\left(k_{2}-k_{1}\right)+1}+\Psi^{2\left(k_{2}-k_{1}\right)} \delta_{p_{k_{2}+1} y}\right) \cdots\left(\Psi^{2\left(k_{l}-k_{l-1}\right)+1}+\Psi^{2\left(k_{l}-k_{l-1}\right)} \delta_{p_{n} y}\right) \\
& \cdot \mathbb{E} \sum_{p_{1}, \ldots, p_{n-1}, i_{n}, j_{n}}\left|\sigma_{i_{n} j_{n}}^{(n)} G_{j_{1} y} \bar{G}_{i_{1} y} V_{1,2} \cdots V_{n-1, n} G_{i_{n} y} \bar{G}_{j_{n} y} / \prod_{t \in \mathcal{T}} t\right|\left|\mathcal{Q}_{y y}\right|^{\alpha+\beta} .
\end{aligned}
$$

To estimate the above, we need to expand

$$
\left(\Psi^{2 k_{1}+1}+\Psi^{2 k_{1}} \delta_{p_{k_{1}+1} y}\right)\left(\Psi^{2\left(k_{2}-k_{1}\right)+1}+\Psi^{2\left(k_{2}-k_{1}\right)} \delta_{p_{k_{2}+1} y}\right) \cdots\left(\Psi^{2\left(k_{l}-k_{l-1}\right)+1}+\Psi^{2\left(k_{l}-k_{l-1}\right)} \delta_{p_{n} y}\right)
$$

and consider each term in the result separately. Here we only give estimates of two terms, and other cases follow in a similar fashion.

Suppose we take the term

$$
\Psi^{2 k_{1}+1} \Psi^{2\left(k_{2}-k_{1}\right)+1} \cdots \Psi^{2\left(k_{l}-k_{l-1}\right)}
$$

from (6.17). Note that $k_{l}=n-1$, and $k_{l}+l=|\mathcal{T}|$. We have

$$
\begin{aligned}
& \left(\frac{1}{\sqrt{L}}\right)^{n+1} \Psi^{2 k_{1}+1} \Psi^{2\left(k_{2}-k_{1}\right)+1} \cdots \Psi^{2\left(k_{l}-k_{l-1}\right)} \cdot \mathbb{E} \sum_{p_{1}, \ldots, p_{n-1}, i_{n}, j_{n}}\left|\sigma_{i_{n} j_{n}}^{(n)} G_{j_{1} y} \bar{G}_{i_{1} y} V_{1,2} \cdots V_{n-1, n} G_{i_{n} y} \bar{G}_{j_{n} y} / \prod_{t \in \mathcal{T}} t\right|\left|\mathcal{Q}_{y y}\right|^{\alpha+\beta} \\
= & \left(\frac{1}{\sqrt{L}}\right)^{n+1} \Psi^{|\mathcal{T}|+n-1} \cdot \mathbb{E} \sum_{p_{1}, \ldots, p_{n-1}, i_{n}, j_{n}}\left|\sigma_{i_{n} j_{n}}^{(n)} G_{j_{1} y} \bar{G}_{i_{1} y} V_{1,2} \cdots V_{n-1, n} G_{i_{n} y} \bar{G}_{j_{n} y} / \prod_{t \in \mathcal{T}} t\right|\left|\mathcal{Q}_{y y}\right|^{\alpha+\beta} \\
\prec & \left(\frac{1}{\sqrt{L}}\right)^{n+1} \Psi^{|\mathcal{T}|+n-1} \Psi \cdot \mathbb{E} \sum_{p_{1}, \ldots, p_{n-1}, q_{n}}\left|G_{j_{1} y} \bar{G}_{i_{1} y} V_{1,2} \cdots V_{n-1, n} G_{i_{n} y} \bar{G}_{j_{n} y} /\right| G_{p_{n} y}\left|\left(\prod_{t \in \mathcal{T}} t\right)\right|\left|\mathcal{Q}_{y y}\right|^{\alpha+\beta} \\
\prec & \left(\frac{1}{\sqrt{L}}\right)^{n+1} \Psi^{|\mathcal{T}|+n-1} \Psi \cdot L^{n}\left(\frac{1}{\sqrt{L \eta}}\right)^{n+1} \Psi^{3 n+1-|\mathcal{T}|-1-(n+1)} \mathbb{E}\left|\mathcal{Q}_{y y}\right|^{\alpha+\beta}=\frac{\Psi^{2}}{L \eta} \cdot\left(\frac{\Psi^{3}}{\sqrt{\eta}}\right)^{n-1} \cdot \mathbb{E}\left|\mathcal{Q}_{y y}\right|^{2 p-n-1},
\end{aligned}
$$

where in the second step we used $\sum_{p_{n}} \sigma_{i_{n} j_{n}}^{(n)}\left|G_{p_{n} y}\right| \prec \Psi$, and in the third step there are at least $n+1$ many $G, \bar{G}$ that we can use to apply Ward identity.

Suppose we take from (6.17) the term

$$
\Psi^{2 k_{1}} \delta_{p_{k_{1}+1} y} \Psi^{2\left(k_{2}-k_{1}\right)+1} \cdots \Psi^{2\left(k_{l}-k_{l-1}\right)}
$$


then we have

$$
\begin{aligned}
&\left(\frac{1}{\sqrt{L}}\right)^{n+1} \Psi^{2 k_{1}} \delta_{p_{k_{1}+1} y} \Psi^{2\left(k_{2}-k_{1}\right)+1} \ldots \Psi^{2\left(k_{l}-k_{l-1}\right)} \\
& \cdot \mathbb{E} \sum_{p_{1}, \ldots, p_{n-1}, i_{n}, j_{n}}\left|\sigma_{i_{n} j_{n}}^{(n)} G_{j_{1} y} \bar{G}_{i_{1} y} V_{1,2} \cdots V_{n-1, n} G_{i_{n} y} \bar{G}_{j_{n} y} / \prod_{t \in \mathcal{T}} t\right|\left|\mathcal{Q}_{y y}\right|^{\alpha+\beta} \\
&=\left(\frac{1}{\sqrt{L}}\right)^{n+1} \Psi^{|\mathcal{T}|+n-2} \cdot \mathbb{E} \sum_{p_{1}, \ldots, p_{n-1}, i_{n}, j_{n}}\left|\delta_{p_{k_{1}+1} y} \sigma_{i_{n} j_{n}}^{(n)} G_{j_{1} y} \bar{G}_{i_{1} y} V_{1,2} \cdots V_{n-1, n} G_{i_{n} y} \bar{G}_{j_{n} y} / \prod_{t \in \mathcal{T}} t\right|\left|\mathcal{Q}_{y y}\right|^{\alpha+\beta} .
\end{aligned}
$$

For $k_{1}+1=n$, we use $\sigma_{i_{n} j_{n}}^{(n)} \prec W^{-1} \prec \Psi^{2}$ and have

$$
\begin{aligned}
&(6.18) \prec\left(\frac{1}{\sqrt{L}}\right)^{n+1} \Psi^{|\mathcal{T}|+n} \cdot \mathbb{E} \sum_{p_{1}, \ldots, p_{n-1}, q_{n}}\left|G_{j_{1} y} \bar{G}_{i_{1} y} V_{1,2} \cdots V_{n-1, n} G_{i_{n} y} \bar{G}_{j_{n} y} /\right| G_{p_{n} y}\left|\left(\prod_{t \in \mathcal{T}} t\right)\right|\left|\mathcal{Q}_{y y}\right|^{\alpha+\beta} \\
& \prec\left(\frac{1}{\sqrt{L}}\right)^{n+1} \Psi^{|\mathcal{T}|+n} \cdot L^{n}\left(\frac{1}{\sqrt{L \eta}}\right)^{n+1} \Psi^{3 n+1-|\mathcal{T}|-1-(n+1)} \mathbb{E}\left|\mathcal{Q}_{y y}\right|^{\alpha+\beta}=\frac{\Psi^{2}}{L \eta} \cdot\left(\frac{\Psi^{3}}{\sqrt{\eta}}\right)^{n-1} \cdot \mathbb{E}\left|\mathcal{Q}_{y y}\right|^{2 p-n-1} .
\end{aligned}
$$

For $k_{1}+1 \leqslant n-1$, note that there is only one factor $\left|G_{p_{k_{1}+1} y}\right|$ in (6.18) that contains the index $p_{k_{1}+1}$, and we have

$$
\begin{aligned}
(6.18) & \prec\left(\frac{1}{\sqrt{L}}\right)^{n+1} \Psi^{|\mathcal{T}|+n-2} \cdot \mathbb{E} \sum_{\substack{p_{1}, \ldots, p_{k_{1}} ; p_{k_{1}+1}=y ; \\
p_{k_{1}+2}, \ldots, p_{n-1}, i_{n}, j_{n}}}\left|\sigma_{i_{n} j_{n}}^{(n)} G_{j_{1} y} \bar{G}_{i_{1} y} V_{1,2} \cdots V_{n-1, n} G_{i_{n} y} \bar{G}_{j_{n} y} /\right| G_{p_{k_{1}+1} y}\left|\left(\prod_{t \in \mathcal{T}} t\right)\right|\left|\mathcal{Q}_{y y}\right|^{\alpha+\beta} \\
\prec & \left.\sum_{\substack{p_{1}, \ldots, p_{k_{1}} ; p_{k_{1}+1}=y \\
p_{k_{1}+2, \ldots, p_{n-1}, q_{n}}}}\right)^{n+1} \Psi^{|\mathcal{T}|+n-1} \cdot \mathbb{E}{\overline{j_{1} y}}_{G_{i_{1} y} V_{1,2} \cdots V_{n-1, n} G_{i_{n} y} \bar{G}_{j_{n} y} /\left.\left|G_{p_{k_{1}+1} y} G_{p_{n} y}\right|\left(\prod_{t \in \mathcal{T}} t\right)|| \mathcal{Q}_{y y}\right|^{\alpha+\beta}} \\
& \prec\left(\frac{1}{\sqrt{L}}\right)^{n+1} \Psi^{|\mathcal{T}|+n-1} \cdot L^{n-1}\left(\frac{1}{\sqrt{L \eta}}\right)^{n} \Psi^{3 n+1-|\mathcal{T}|-2-n} \mathbb{E}\left|\mathcal{Q}_{y y}\right|^{\alpha+\beta} \prec \frac{\Psi^{2}}{L \eta} \cdot\left(\frac{\Psi^{3}}{\sqrt{\eta}}\right)^{n-1} \cdot \mathbb{E}\left|\mathcal{Q}_{y y}\right|^{2 p-n-1},
\end{aligned}
$$

where in the last step we used the estimate $\frac{\sqrt{L \eta}}{L \Psi} \prec 1$.

One can take other bounds in (6.17) and show that we have the same bound. Thus we obtained from (6.16) that

$$
(A) \prec \frac{\Psi^{2}}{L \eta} \cdot\left(\frac{\Psi^{3}}{\sqrt{\eta}}\right)^{n-1} \cdot \mathbb{E}\left|\mathcal{Q}_{y y}\right|^{2 p-n-1} \prec\left(\frac{\Psi}{\sqrt{L \eta}}+\frac{\Psi^{3}}{\sqrt{\eta}}\right)^{n+1} \mathbb{E}\left|\mathcal{Q}_{y y}\right|^{2 p-n-1} .
$$

Step 3. Now let's look at the first and fourth term on the RHS of (6.12), which is (A) and (D). It is important to consider these two contributions together because there is a crucial cancellation between them. By writing

$$
\mathcal{Q}_{j_{n} y}=\sum_{i_{n+1}, j_{n+1}} S_{\mathbf{v} i}\left(H_{i_{n+1} j_{n+1}} G_{j_{n+1} j_{n}} \bar{G}_{i_{n+1} y}+S_{i_{n+1} j_{n+1}} G_{j_{n+1} j_{n+1}} G_{i_{n+1} x} \bar{G}_{i_{n+1} y}+S_{i_{n+1} j_{n+1}} \bar{G}_{i_{n+1} i_{n+1}} G_{j_{n+1} j_{n}} \bar{G}_{j_{n+1} y}\right)
$$

and using cumulant expansion on $H_{i_{n+1} j_{n+1}}$ we see that

$$
\begin{aligned}
& (A)+(D)=-\left(\frac{1}{\sqrt{L}}\right)^{n} \mathbb{E} \sum_{i_{1}, j_{1}, \ldots, i_{n}, j_{n}} \sigma_{i_{1} j_{1}}^{(1)} \cdots \sigma_{i_{n} j_{n}}^{(n)} G_{j_{1} y} \bar{G}_{i_{1} y} V_{1,2} \cdots V_{n-1, n} \mathcal{Q}_{j_{n} y} G_{i_{n} y} \cdot \mathcal{Q}_{y y}^{\alpha} \overline{\mathcal{Q}}_{y y}^{\beta}+(D) \\
= & -\left(\frac{1}{\sqrt{L}}\right)^{n} \mathbb{E} \sum_{i_{1}, j_{1}, \ldots, i_{n+1}, j_{n+1}} \sigma_{i_{1} j_{1}}^{(1)} \cdots \sigma_{i_{n} j_{n}}^{(n)} \partial_{j_{n+1} i_{n+1}}\left(G_{j_{1} y} \bar{G}_{i_{1} y} V_{1,2} \cdots V_{n-1, n} S_{\mathbf{v} i_{n+1}} S_{i_{n+1} j_{n+1}} G_{j_{n+1} j_{n}} \bar{G}_{i_{n+1} y} G_{i_{n} y} \cdot \mathcal{Q}_{y y}^{\alpha} \overline{\mathcal{Q}}_{y y}^{\beta}\right) \\
& -\left(\frac{1}{\sqrt{L}}\right)^{n} \mathbb{E} \sum_{i_{1}, j_{1}, \ldots, i_{n}, j_{n}} \sigma_{i_{1} j_{1}}^{(1)} \cdots \sigma_{i_{n} j_{n}}^{(n)} G_{j_{1} y} \bar{G}_{i_{1} y} V_{1,2} \cdots V_{n-1, n} . \\
& \left(\sum_{i_{n+1}, j_{n+1}} S_{\mathbf{v} i_{n+1}} S_{i_{n+1} j_{n+1}}\left(G_{j_{n+1} j_{n+1}} G_{i_{n+1} j_{n}} \bar{G}_{i_{n+1} y}+G_{j_{n+1} j_{n}} \bar{G}_{i_{n+1} i_{n+1}} \bar{G}_{j_{n+1} y}\right)\right) G_{i_{n} y} \mathcal{Q}_{y y}^{\alpha} \overline{\mathcal{Q}}_{y y}^{\beta}+(D)
\end{aligned}
$$


We see that when the differential $\partial_{j_{n+1} i_{n+1}}$ is applied to $G_{j_{n+1} j_{n}}, \bar{G}_{i_{n+1} y}$, and $G_{i_{n} y}$, the result will cancel the second, third and last term on the RHS of (6.20) respectively. Thus by setting $\sigma_{i_{n+1} j_{n+1}}^{(n+1)}:=\sqrt{L} S_{\mathbf{v} i_{n+1}} S_{i_{n+1} j_{n+1}}$ we have

$$
\begin{aligned}
& (A)+(D) \\
= & -\left(\frac{1}{\sqrt{L}}\right)^{n+1} \mathbb{E} \sum_{i_{1}, j_{1}, \ldots, i_{n+1}, j_{n+1}} \sigma_{i_{1} j_{1}}^{(1)} \cdots \sigma_{i_{n+1} j_{n+1}}^{(n+1)}\left[\partial_{j_{n+1} i_{n+1}}\left(G_{j_{1} y} \bar{G}_{i_{1} y} V_{1,2} \cdots V_{n-1, n} \cdot \mathcal{Q}_{y y}^{\alpha} \overline{\mathcal{Q}}_{y y}^{\beta}\right)\right] G_{j_{n+1} j_{n}} \bar{G}_{i_{n+1} y} G_{i_{n} y} .
\end{aligned}
$$

Also, note that $V_{n, n+1}:=G_{j_{n+1} j_{n}} \bar{G}_{i_{n+1} y} G_{i_{n} y} \in \mathcal{V}_{n, n+1}$, thus when the differential is applied to $\mathcal{Q}_{y y}^{\alpha} \overline{\mathcal{Q}}_{y y}^{\beta}$, we return to the same form as (6.11). The term left to be estimated is

$$
-\left(\frac{1}{\sqrt{L}}\right)^{n+1} \mathbb{E} \sum_{i_{1}, j_{1}, \ldots, i_{n+1}, j_{n+1}} \sigma_{i_{1} j_{1}}^{(1)} \cdots \sigma_{i_{n+1} j_{n+1}}^{(n+1)}\left[\partial_{j_{n+1} i_{n+1}}\left(G_{j_{1} y} \bar{G}_{i_{1} y} V_{1,2} \cdots V_{n-1, n}\right)\right] G_{j_{n+1} j_{n}} \bar{G}_{i_{n+1} y} G_{i_{n} y} \cdot \mathcal{Q}_{y y}^{\alpha} \overline{\mathcal{Q}}_{y y}^{\beta} .
$$

As in the estimation of (A), we can first apply Lemma 6.1 to sum over $q_{1}, \ldots, q_{n-1}, j_{n}, q_{n+1}$. Depending on whether $\partial_{j_{n+1} i_{n+1}}$ is applied to a $G$ or $\bar{G}$, the index $q_{n+1}$ will be equal to $j_{n+1}$ or $i_{n+1}$ respectively. Also, in this case we will have one loop (in the sense of Remark 6.2) if the differential is applied to a $G_{j_{k} x}\left(x \in\left\{y, j_{k-1}\right\}\right.$ ) and we have $G_{j_{n+1} j_{n}} \cdots G_{j_{k+1} j_{k}}$ in (6.22). Other summations in (6.22) will still give rise to chains. Thus, with the same procedure of Step 2 we can use Lemma 6.1(i)(ii) to show that

$$
(6.22) \prec\left(\frac{\Psi}{L \eta}+\frac{\Psi^{3}}{\sqrt{\eta}}\right)^{n+1} \cdot \mathbb{E}\left|\mathcal{Q}_{y y}\right|^{2 p-n-1} .
$$

Thus by (6.21)-(6.23) we have

$$
\begin{array}{r}
(A)+(D)=\left(\frac{1}{\sqrt{L}}\right)^{n+1} \mathbb{E} \sum_{i_{1}, j_{1}, \ldots, i_{n+1}, j_{n+1}} \sigma_{i_{1} j_{1}}^{(1)} \cdots \sigma_{i_{n+1} j_{n+1}}^{(n+1)} G_{j_{1} y} \bar{G}_{i_{1} y} V_{1,2} \cdots V_{n, n+1}\left(\partial_{j_{n+1} i_{n+1}} \mathcal{Q}_{y y}^{\alpha} \overline{\mathcal{Q}}_{y y}^{\beta}\right) \\
+O_{\prec}\left(\frac{\Psi}{L \eta}+\frac{\Psi^{3}}{\sqrt{\eta}}\right)^{n+1} \cdot \mathbb{E}\left|\mathcal{Q}_{y y}\right|^{2 p-n-1} .
\end{array}
$$

Similarly,

$$
\begin{array}{r}
(B)+(E)=\left(\frac{1}{\sqrt{L}}\right)^{n+1} \mathbb{E} \sum_{i_{1}, j_{1}, \ldots, i_{n+1}, j_{n+1}} \sigma_{i_{1} j_{1}}^{(1)} \cdots \sigma_{i_{n+1} j_{n+1}}^{(n+1)} G_{j_{1} y} \bar{G}_{i_{1} y} V_{1,2} \cdots V_{n-1, n} V_{n, n+1}^{\prime}\left(\partial_{j_{n+1} i_{n+1}} \mathcal{Q}_{y y}^{\alpha} \overline{\mathcal{Q}}_{y y}^{\beta}\right) \\
+O \prec\left(\frac{\Psi}{L \eta}+\frac{\Psi^{3}}{\sqrt{\eta}}\right)^{n+1} \cdot \mathbb{E}\left|\mathcal{Q}_{y y}\right|^{2 p-n-1},
\end{array}
$$

where $V_{n, n+1}^{\prime}:=\bar{G}_{i_{n+1} i_{n}} \bar{G}_{j_{n} y} G_{j_{n+1} y} \in \mathcal{V}_{n, n+1}$.

Step 4. Plugging (6.19), (6.24) and (6.25) into (6.12) gives

$$
\begin{aligned}
&\left(\frac{1}{\sqrt{L}}\right)^{n} \mathbb{E} \sum_{i_{1}, j_{1}, \ldots, i_{n}, j_{n}} \sigma_{i_{1} j_{1}}^{(1)} \cdots \sigma_{i_{n} j_{n}}^{(n)} G_{j_{1} y} \bar{G}_{i_{1} y} V_{1,2} \cdots V_{n-1, n}\left(\partial_{j_{n} i_{n}} \mathcal{Q}_{y y}\right) \mathcal{Q}_{y y}^{\alpha} \overline{\mathcal{Q}}_{y y}^{\beta} \\
&=\left(\frac{1}{\sqrt{L}}\right)^{n+1} \mathbb{E} \sum_{i_{1}, j_{1}, \ldots, i_{n+1}, j_{n+1}} \sigma_{i_{1} j_{1}}^{(1)} \cdots \sigma_{i_{n+1} j_{n+1}}^{(n+1)} G_{j_{1} y} \bar{G}_{i_{1} y} V_{1,2} \cdots V_{n-1, n}\left(V_{n, n+1}+V_{n, n+1}^{\prime}\right)\left(\partial_{j_{n+1} i_{n+1}} \mathcal{Q}_{y y}^{\alpha} \overline{\mathcal{Q}}_{y y}^{\beta}\right) \\
&+O \prec\left(\frac{\Psi}{L \eta}+\frac{\Psi^{3}}{\sqrt{\eta}}\right)^{n+1} \cdot \mathbb{E}\left|\mathcal{Q}_{y y}\right|^{2 p-n-1} \cdot \quad(6 .
\end{aligned}
$$

Similar result can also be obtained for

$$
\left(\frac{1}{\sqrt{L}}\right)^{n} \mathbb{E} \sum_{i_{1}, j_{1}, \ldots, i_{n}, j_{n}} \sigma_{i_{1} j_{1}}^{(1)} \cdots \sigma_{i_{n} j_{n}}^{(n)} G_{j_{1} y} \bar{G}_{i_{1} y} V_{1,2} \cdots V_{n-1, n}\left(\partial_{j_{n} i_{n}} \overline{\mathcal{Q}}_{y y}\right) \mathcal{Q}_{y y}^{\alpha} \overline{\mathcal{Q}}_{y y}^{\beta}
$$


together with (6.9) we have

$$
\mathbb{E}\left|\mathcal{Q}_{y y}\right|^{2 p}=\sum_{n=1}^{2 p-1} O_{\prec}\left(\frac{\Psi}{L \eta}+\frac{\Psi^{3}}{\sqrt{\eta}}\right)^{n+1} \cdot \mathbb{E}\left|\mathcal{Q}_{y y}\right|^{2 p-n-1},
$$

which gives the desired result by Lemma 6.3.

The proof of (5.9) proceeds as the one for (5.8) but the $S_{\mathbf{v} i_{m}}$ 's $(m=1, \ldots, 2 p-1)$ are replaced by the $S_{x i_{m}}$ 's which are bounded by $W^{-1}$. Finally, (5.10) and (5.11) are easily obtained by using Lemma 6.1 (iii) and the Ward identity (6.7).

\section{$7 \quad$ Proof of Lemma 6.1}

In order to prove Lemma 6.1 we will need some auxiliary technical lemmata. The first one concerns a trick to write self-consistent equations for the kind of expectations we are going to use.

Lemma 7.1. Consider the expectation

$$
D_{a b c}=\mathbb{E} \sum_{i} \sigma_{a i} G_{i b} G_{c i} \mathfrak{p}(G, \bar{G})
$$

where $\sigma \in \mathbb{S}$ and $\mathfrak{p}(G, \bar{G})$ is a polynomial of $G_{x y}$ and $\bar{G}_{x^{\prime} y^{\prime}}$ with $x, y, x^{\prime}, y^{\prime} \not \equiv i$. Then

$$
\begin{aligned}
D_{a b c}= & \mathbb{E} \xi_{a b} G_{c b} \mathfrak{p}(G, \bar{G})-\mathbb{E} \sum_{i, j} \xi_{a i} S_{i j} G_{j b} G_{c i} \partial_{j i} \mathfrak{p}(G, \bar{G}) \\
& +\mathbb{E} \sum_{i, j} \xi_{a i} S_{i j}\left(\left(G_{j j}-\mathfrak{m}\right) G_{i b} G_{c i}+G_{j b} G_{c j}\left(G_{i i}-\mathfrak{m}\right)\right) \mathfrak{p}(G, \bar{G})
\end{aligned}
$$

where $\xi \in \mathbb{S}$.

Proof. Applying (5.4) and the cumulant expansion formula (5.6), we get:

$$
z D_{a b c}=\mathbb{E} \sum_{i} \sigma_{a i}\left(z G_{i b}\right) G_{c i} \mathfrak{p}(G, \bar{G})=-\mathbb{E} \sum_{i} \sigma_{a i} \delta_{i b} G_{c i} \mathfrak{p}(G, \bar{G})+\mathbb{E} \sum_{i, j} \sigma_{a i} S_{i j} \partial_{j i}\left(G_{j b} G_{c i} \mathfrak{p}(G, \bar{G})\right)
$$

By performing the derivatives and writing the diagonal entries of $G$ as $\left(G_{i i}-\mathfrak{m}\right)+\mathfrak{m}$ one has

$$
\begin{aligned}
z D_{a b c}=-\mathbb{E} \sigma_{a b} G_{c b} \mathfrak{p}(G, \bar{G})-\mathbb{E} \sum_{i, j} \sigma_{a i} S_{i j}\left(G_{j j} G_{i b} G_{c i}+G_{j b} G_{c j} G_{i i}\right) \mathfrak{p}(G, \bar{G})+\mathbb{E} \sum_{i, j} \sigma_{a i} S_{i j} G_{j b} G_{c i} \partial_{j i} \mathfrak{p}(G, \bar{G}) \\
=-\mathbb{E} \sigma_{a b} G_{c b} \mathfrak{p}(G, \bar{G})-\mathfrak{m} D_{a b c}-\mathfrak{m} \sum_{i} \sigma_{a i} \mathbb{E} \sum_{j} S_{i j} G_{j b} G_{c j} \mathfrak{p}(G, \bar{G}) \\
\quad-\mathbb{E} \sum_{i, j} \sigma_{a i} S_{i j}\left(\left(G_{j j}-\mathfrak{m}\right) G_{i b} G_{c i}+G_{j b} G_{c j}\left(G_{i i}-\mathfrak{m}\right)\right) \mathfrak{p}(G, \bar{G})+\mathbb{E} \sum_{i, j} \sigma_{a i} S_{i j} G_{j b} G_{c i} \partial_{j i} \mathfrak{p}(G, \bar{G}) .
\end{aligned}
$$

Let us consider the summation of the last term of the expansion in (7.3)

$$
\tilde{D}_{a b c}:=\mathbb{E} \sum_{i} S_{a i} G_{i b} G_{c i} \mathfrak{p}(G, \bar{G}) .
$$

Note that the only difference between $D_{a b c}$ and $\tilde{D}_{a b c}$ is that $\sigma_{a i}$ in $D_{a b c}$ is replaced by $S_{a i}$ in $\tilde{D}_{a b c}$. Expanding $\tilde{D}_{a b c}$ in the same way we have done for $D_{a b c}$, we get

$$
\begin{aligned}
& \sum_{i}\left((z+m) \delta_{a i}+\mathfrak{m} S_{a i}\right) \tilde{D}_{i b c}=-\mathbb{E} S_{a b} G_{c b} \mathfrak{p}(G, \bar{G})+\mathbb{E} \sum_{i, j} S_{a i} S_{i j} G_{j b} G_{c i} \partial_{j i} \mathfrak{p}(G, \bar{G}) \\
& -\mathbb{E} \sum_{i, j} S_{a i} S_{i j}\left(\left(G_{j j}-\mathfrak{m}\right) G_{i b} G_{c i}+G_{j b} G_{c j}\left(G_{i i}-\mathfrak{m}\right)\right) \mathfrak{p}(G, \bar{G}) .
\end{aligned}
$$


Let us analyse the operator on the left hand side of (7.4): thanks to (2.10) we get

$$
\mathcal{L}:=((z+\mathfrak{m}) I+\mathfrak{m} S)^{-1}=-\mathfrak{m}\left(I-\mathfrak{m}^{2} S\right)^{-1} .
$$

Since we are interested in the bulk spectrum of the band matrices, from Proposition B.2 in [7] we see that there is a positive constant $C$ such that

$$
\rho:=\|\mathcal{L}\|_{\infty \rightarrow \infty} \leqslant C \log L \prec 1 .
$$

From the translational invariance of $\mathcal{L}$ and (7.6) one can easily see that $\mathcal{L} \xi \in \mathbb{S}$ for any $\xi \in \mathbb{S}$. Thus, (7.6) and (7.4) imply

$$
\begin{aligned}
\tilde{D}_{a b c}= & -\mathbb{E} \tau_{a b} G_{c b} \mathfrak{p}(G, \bar{G})+\mathbb{E} \sum_{i, j} \tau_{a i} S_{i j} G_{j b} G_{c i} \partial_{j i} \mathfrak{p}(G, \bar{G}) \\
& -\mathbb{E} \sum_{i, j} \tau_{a i} S_{i j}\left(\left(G_{j j}-\mathfrak{m}\right) G_{i b} G_{c i}+G_{j b} G_{c j}\left(G_{i i}-\mathfrak{m}\right)\right) \mathfrak{p}(G, \bar{G})
\end{aligned}
$$

where $\tau=\mathcal{L} S \in \mathbb{S}$. Coming back to (7.3) and using again (5.4), we get

$$
\begin{aligned}
& D_{a b c}=\mathfrak{m} \mathbb{E} \sigma_{a b} G_{c b} \mathfrak{p}(G, \bar{G})-\mathfrak{m} \mathbb{E} \sum_{i, j} \sigma_{a i} S_{i j} G_{j b} G_{c i} \partial_{j i} \mathfrak{p}(G, \bar{G}) \\
& +\mathfrak{m} \mathbb{E} \sum_{i, j} \sigma_{a i} S_{i j}\left(\left(G_{j j}-\mathfrak{m}\right) G_{i b} G_{c i}+G_{j b} G_{c j}\left(G_{i i}-\mathfrak{m}\right)\right) \mathfrak{p}(G, \bar{G})+\mathfrak{m}^{2} \sum_{i} \sigma_{a i} \tilde{D}_{i b c} .
\end{aligned}
$$

In conclusion, using (7.7) and the fact that $\mathbb{S}$ is close with respect to matrix addition and multiplication, we get

$$
\begin{aligned}
D_{a b c}= & \mathbb{E} \xi_{a b} G_{c b} \mathfrak{p}(G, \bar{G})-\mathbb{E} \sum_{i, j} \xi_{a i} S_{i j} G_{j b} G_{c i} \partial_{j i} \mathfrak{p}(G, \bar{G}) \\
& +\mathbb{E} \sum_{i, j} \xi_{a i} S_{i j}\left(\left(G_{j j}-\mathfrak{m}\right) G_{i b} G_{c i}+G_{j b} G_{c j}\left(G_{i i}-\mathfrak{m}\right)\right) \mathfrak{p}(G, \bar{G}) .
\end{aligned}
$$

where $\xi=\mathfrak{m}(\sigma-\mathfrak{m} \sigma \tau) \in \mathbb{S}$.

It is convenient to define the following transformation on the matrices belonging to $\mathbb{S}$ : let $\sigma \in \mathbb{S}$, then we set

$$
\breve{\sigma}:=\mathfrak{m}(\sigma-\mathfrak{m} \sigma \mathcal{L} S) \in \mathbb{S}
$$

where $\mathcal{L}$ is the matrix defined in (7.5). With this notation we can write $\xi$ in Lemma 7.1 as $\xi=\breve{\sigma}$.

By using an argument very similar to the one exploited in Lemma 7.1 we can also show that

$$
\mathcal{G}_{a}:=\sum_{i} \sigma_{a i} \mathbb{E}\left(G_{i i}-\mathfrak{m}\right) \prec \Psi^{2}
$$

under the assumption that $\Lambda \prec \Psi$. In fact, by the cumulant expansion formula (5.6) we have

$$
z \mathcal{G}_{a}=-\mathbb{E} \sum_{i, j} \sigma_{a i} S_{i j}\left(G_{j j}-\mathfrak{m}\right)\left(G_{i i}-\mathfrak{m}\right)-\mathfrak{m} \mathcal{G}_{a}-\mathfrak{m} \sum_{i} \sigma_{a i} \tilde{\mathcal{G}}_{i}
$$

where $\tilde{\mathcal{G}}_{a}:=\sum_{i} S_{a i} \mathbb{E}\left(G_{i i}-\mathfrak{m}\right)$. Expanding $\tilde{\mathcal{G}}_{a}$ again via (5.6) and using the properties of the operator $\mathcal{L}$ as we did for $\tilde{D}_{a b c}$ in the proof of Lemma 7.1, we get

$$
\tilde{\mathcal{G}}_{a}=-\mathbb{E} \sum_{i, j}(\mathcal{L} S)_{a i} S_{i j}\left(G_{j j}-\mathfrak{m}\right)\left(G_{i i}-\mathfrak{m}\right) .
$$

Using (7.9), this implies $\mathcal{G}_{a}=\mathbb{E} \sum_{i, j} \breve{\sigma}_{a i} S_{i j}\left(G_{j j}-\mathfrak{m}\right)\left(G_{i i}-\mathfrak{m}\right) \prec \Psi^{2}$. In the same way one gets also the following estimates

$$
\mathbb{E}\left(G_{j j}-\mathfrak{m}\right) \prec \Psi^{2}, \quad \mathbb{E} G_{j k} \prec \Psi^{2} \text { for } k \neq j, \quad \sum_{j} \sigma_{i j} \mathbb{E} G_{j k} \prec \Psi^{2} .
$$

We will prove (6.3) and (6.4) by induction over $n$. Therefore, first we should check that those two formulae are true for $n=1$ and $n=2$ respectively. This is shown in the following lemma. 
Lemma 7.2. Under the assumption of Lemma 6.1, for some $\xi \in \mathbb{S}$ with nonnegative entries one has

$$
\begin{aligned}
& Y_{a b ; u}^{(1)} \prec \Psi^{3}+\delta_{a b} \Psi^{2}, \\
& Z_{a b}^{(2)} \prec \Psi^{4}+\xi_{a b} .
\end{aligned}
$$

Proof. We slightly simplify the notation by setting $Y_{u a b}:=Y_{a b ; u}^{(1)}=\sum_{i} \sigma_{u i} G_{a i} G_{i b}$ with $\sigma \in \mathbb{S}$. We fix an arbitrary integer $p \geqslant 1$ and we apply Lemma 7.1 to $\mathbb{E}\left|Y_{u a b}\right|^{2 p}=\mathbb{E} \sum_{i} \sigma_{u i} G_{a i} G_{i b} Y_{s a b}^{p-1} \bar{Y}_{s a b}^{p}$, so we get

$$
\begin{aligned}
& \mathbb{E}\left|Y_{u a b}\right|^{2 p} \prec\left|\mathbb{E} \breve{\sigma}_{u a} G_{a b}\left(Y_{u a b}\right)^{p-1}\left(\bar{Y}_{u a b}\right)^{p}\right|+\left|\mathbb{E} \sum_{i, j} \breve{\sigma}_{u i} S_{i j} G_{a i} G_{j b} \partial_{j i}\left(Y_{u a b}^{p-1} \bar{Y}_{u a b}^{p}\right)\right| \\
& +\left|\mathbb{E} \sum_{i, j} \breve{\sigma}_{u i} S_{i j}\left(\left(G_{j j}-\mathfrak{m}\right) G_{a i} G_{i b}+G_{a j} G_{j b}\left(G_{i i}-\mathfrak{m}\right)\right) Y_{u a b}^{p-1} \bar{Y}_{u a b}^{p}\right| \\
& \prec\left(\Psi^{3}+\delta_{a b} \Psi^{2}\right) \mathbb{E}\left|Y_{u a b}\right|^{2 p-1}+\left|\mathbb{E} \sum_{i, j} \breve{\sigma}_{u i} S_{i j} G_{a j} G_{i b} \partial_{j i}\left(Y_{u a b}^{p-1} \bar{Y}_{u a b}^{p}\right)\right|
\end{aligned}
$$

where we used the hypothesis $\Lambda \prec \Psi$. Let us focus on the last term of (7.13): we note that

$$
\partial_{j i} Y_{u a b}=-G_{i a} Y_{u j b}-G_{b j} Y_{u a i}, \quad \partial_{j i} \bar{Y}_{u a b}=-\bar{G}_{j a} \bar{Y}_{u i b}-\bar{G}_{b i} \bar{Y}_{u a j} .
$$

For $a \neq b$ we set the a prior bound

$$
Y_{u a b} \prec \lambda
$$

for $\lambda \in\left[\Psi^{3}, L^{C}\right]$ for some $C>0$, so that for $a \neq b$, recalling the trivial bound $Y_{u a b} \prec \Psi^{2}$, using (7.15), (7.14) and (7.13), we have

$$
\mathbb{E}\left|Y_{\text {uab }}\right|^{2 p} \prec \Psi^{3} \mathbb{E}\left|Y_{\text {uab }}\right|^{2 p-1}+\lambda \Psi^{3} \mathbb{E}\left|Y_{\text {uab }}\right|^{2 p-2}+\Psi^{6} \mathbb{E}\left|Y_{\text {uab }}\right|^{2 p-2} \prec \lambda \Psi^{3} \mathbb{E}\left|Y_{\text {uab }}\right|^{2 p-2} .
$$

From Lemma 6.3 with $\vartheta=\lambda, \varphi=\Psi^{3}$ and $q=1 / 2$ we deduce that $Y_{u a b}=Y_{a b ; u}^{(1)} \prec \Psi^{3}$ for $a \neq b$, and therefore in general we obtain

$$
Y_{a b ; u}^{(1)} \prec \Psi^{3}+\delta_{a b} \Psi^{2} .
$$

Let us now prove (7.12) with the same strategy used for (7.11). We recall that

$$
Z_{a b}^{(2)}:=\sum_{i j} \sigma_{a i} \tau_{b j} G_{i j} G_{j i}
$$

with $\sigma, \tau \in \mathbb{S}$. In the following we will drop the superscript (2). Again from Lemma 7.1 for any fixed integer $p$ we have

$$
\begin{aligned}
\mathbb{E}\left|Z_{a b}\right|^{2 p} \prec & \left|\mathbb{E} \sum_{i} \breve{\sigma}_{a i} \tau_{b i} G_{i i} Z_{a b}^{p-1} \bar{Z}_{a b}^{p}\right|+\left|\mathbb{E} \sum_{i, j, k} \breve{\sigma}_{a i} \tau_{b j} S_{i k}\left[\left(G_{k k}-\mathfrak{m}\right) G_{j i} G_{i j}+\left(G_{i i}-\mathfrak{m}\right) G_{k j} G_{j k}\right] Z_{a b}^{p-1} \bar{Z}_{a b}^{p}\right| \\
& +\left|\mathbb{E} \sum_{i, j, k} \breve{\sigma}_{a i} \tau_{b j} S_{i k} G_{k j} G_{j i} \partial_{k i}\left(Z_{a b}^{p-1} \bar{Z}_{a b}^{p}\right)\right| \\
& \prec\left(\xi_{a b}+\left|B_{a b}\right|+\Psi^{4}\right) \mathbb{E}\left|Z_{c d}\right|^{2 p-1}+\left|\mathbb{E} \sum_{i, j, k} \breve{\sigma}_{a i} \tau_{b j} S_{i k} G_{k j} G_{j i} \partial_{k i}\left(Z_{a b}^{p-1} \bar{Z}_{a b}^{p}\right)\right|
\end{aligned}
$$

where $\xi \in \mathbb{S}$ and in the last line we used (6.5). Moreover,

$$
B_{a b}:=\sum_{i, j, k} \breve{\sigma}_{a i} \tau_{b j} S_{i k} G_{i i}^{b} G_{k j} G_{j k}
$$


where we used the notation $g^{b}:=g-\mathbb{E} g$. We now have to estimate $B_{a b}$ : note that by using (7.11) we get $B_{a b} \prec \Psi W^{-1}$, but this is not enough for our purposes. Thus, we are going to expand $B_{a b}$ : as before Lemma 7.1 implies

$$
\begin{aligned}
& \mathbb{E}\left|B_{a b}\right|^{2 p} \prec\left|\mathbb{E} \sum_{i, j} \breve{\sigma}_{a i} \breve{\tau}_{b j} S_{i j} G_{i i}^{b} G_{j j} B_{a b}^{p-1} \bar{B}_{a b}^{p}\right|+\left|\mathbb{E} \sum_{i, j, k, l} \breve{\sigma}_{a i} \breve{\tau}_{b j} S_{i k} S_{j l} G_{k j} G_{l k} \partial_{l j}\left(G_{i i}^{b} B_{a b}^{p-1} \bar{B}_{a b}^{p}\right)\right| \\
& \prec \Psi^{4} \mathbb{E}\left|B_{a b}\right|^{2 p-1}+\Psi^{8} \mathbb{E}\left|B_{a b}\right|^{2 p-2}
\end{aligned}
$$

where in the second line we used (6.5), (7.11) and the fact that trivially one gets $\partial_{l j} B_{a b} \prec \Psi^{4}$. Thus, we obtained that

$$
B_{a b} \prec \Psi^{4}
$$

and

$$
\mathbb{E}\left|Z_{a b}\right|^{2 p} \prec\left(\xi_{a b}+\Psi^{4}\right) \mathbb{E}\left|Z_{a b}\right|^{2 p-1}+\left|\mathbb{E} \sum_{i, j, k} \breve{\sigma}_{a i} \tau_{b j} S_{i k} G_{k j} G_{j i} \partial_{k i}\left(Z_{a b}^{p-1} \bar{Z}_{a b}^{p}\right)\right| .
$$

Let us focus on the last term in (7.21): we need to compute the derivatives of $Z$ :

$$
\begin{aligned}
\partial_{k i} Z_{c d} & =-\sum_{l h} \sigma_{c l} \tau_{d h}\left(G_{l k} G_{i h} G_{h l}+G_{h k} G_{i l} G_{l h}\right) \\
\partial_{k i} \bar{Z}_{c d} & =-\sum_{l h} \bar{\sigma}_{c l} \bar{\tau}_{d h}\left(\bar{G}_{h l} \bar{G}_{l i} \bar{G}_{k h}+\bar{G}_{l h} \bar{G}_{h i} \bar{G}_{k l}\right) .
\end{aligned}
$$

We consider the first term in (7.22) (the second one is treated similarly as well as the two terms in (7.23)): we define

$$
A_{a b c d}:=\sum_{i, j} \sigma_{a i} \tau_{b j} G_{i c} G_{d j} G_{j i}
$$

To bound $A_{a b c d}$ we perform again a cumulant expansions. For $c \neq d$ set the prior bound

$$
A_{a b c d} \prec \alpha
$$

with $\alpha \in\left[\Psi^{5}, L^{C}\right]$. For any integer $p \geqslant 1$ Lemma 7.1 yields

$$
\begin{aligned}
& \mathbb{E}\left|A_{a b c d}\right|^{2 p} \prec\left|\mathbb{E} \sum_{j} \breve{\sigma}_{a c} \tau_{b j} G_{c c} G_{d j} G_{j c} A_{a b c d}^{p-1} \bar{A}_{a b c d}^{p}\right| \\
& +\left|\mathbb{E} \sum_{i, j, k} \breve{\sigma}_{a i} \tau_{b j} S_{k i}\left[\left(G_{k k}-\mathfrak{m}\right) G_{i c} G_{d j} G_{j i k}+\left(G_{i i}-\mathfrak{m}\right) G_{k c} G_{d j} G_{j k}\right] A_{a b c d}^{p-1} \bar{A}_{a b c d}^{p}\right| \\
& +\left|\mathbb{E} \sum_{i, j, k} \breve{\sigma}_{a i} \tau_{b j} S_{k i} G_{k c} G_{d k} G_{i j} G_{j i} A_{a b c d}^{p-1} \bar{A}_{a b c d}^{p}\right|+\left|\mathbb{E} \sum_{i, j, k} \breve{\sigma}_{a i} \tau_{b j} S_{k i} G_{k c} G_{d j} G_{j i} \partial_{k i}\left(A_{a b c d}^{p-1} \bar{A}_{a b c d}^{p}\right)\right| .
\end{aligned}
$$

By using (7.11) we get for $c \neq d$

$$
\mathbb{E}\left|A_{a b c d}\right|^{2 p} \prec \Psi^{5} \mathbb{E}\left|A_{a b c d}\right|^{2 p-1}+\left|\mathbb{E} \sum_{i, j, k} \breve{\sigma}_{a i} \tau_{b j} S_{k i} G_{k c} G_{d j} G_{j i} \partial_{k i}\left(A_{a b c d}^{p-1} \bar{A}_{a b c d}\right)^{p}\right| .
$$

To bound the second term on the right hand side of (7.26) we observe that

$$
\begin{aligned}
& \partial_{k i} A_{a b c d}=-G_{i c} A_{a b k d}-G_{d k} A_{a b c i}-\sum_{l h} \sigma_{a l} \tau_{b h} G_{l d} G_{c h} G_{h k} G_{i l} \\
& \prec\left|G_{i c}\right|\left(\alpha+\delta_{k d}\left|A_{a b d d}\right|\right)+\left|G_{d k}\right|\left(\alpha+\delta_{c i}\left|A_{a b c c}\right|\right)+\left(\Psi^{3}+\delta_{d l} W^{-1}\right)\left(\Psi^{3}+\delta_{c k} W^{-1}\right) \\
& \prec\left|G_{i c}\right|\left(\alpha+\delta_{k d} \Psi^{4}\right)+\left|G_{d k}\right|\left(\alpha+\delta_{c i} \Psi^{4}\right)+\left(\Psi^{3}+\delta_{d i} W^{-1}\right)\left(\Psi^{3}+\delta_{c k} W^{-1}\right)
\end{aligned}
$$


where we used (7.11), the trivial estimate $A_{a b c d} \prec \Psi^{4}$ (where we exploited again (7.11)) and the prior estimate (7.24). Note that a similar bound holds for $\partial_{k i} \bar{A}_{a b c d}$. Thus, using (7.27) and (7.11), we get

$$
\mathbb{E} \sum_{i, j, k} \breve{\sigma}_{a i} \tau_{b j} S_{k i} G_{k c} G_{d j} G_{j i} \partial_{k i}\left(A_{a b c d}^{p-1} \bar{A}_{a b c d}^{p}\right) \prec\left(\alpha \Psi^{5}+\Psi^{10}\right) \mathbb{E}\left|A_{a b c d}\right|^{2 p-2}
$$

which implies, via Lemma 6.3 , that for $c \neq d$ we have $A_{a b c d} \prec \Psi^{5}$ and in general

$$
A_{a b c d} \prec \Psi^{5}+\delta_{c d} \Psi^{4} .
$$

Thus, by using (7.28) and (7.11) in (7.17) we get

$$
\mathbb{E}\left|Z_{a b}\right|^{2 p} \prec\left(\xi_{a b}+\Psi^{4}\right) \mathbb{E}\left|Z_{a b}\right|^{2 p-1}+\Psi^{8} \mathbb{E}\left|Z_{a b}\right|^{2 p-2} .
$$

Therefore, Lemma 6.3 implies that $Z_{a b} \prec \xi_{a b}+\Psi^{4}$.

7.1. Chain estimates: proof of (6.3). We will proceed by induction on $n$ : thanks to (7.11), we know that $Y_{a b ; u}^{(1)} \prec \Psi^{3}+\delta_{a b} \Psi^{2}$ and we assume as induction hypothesis that

$$
Y_{a b ; u_{1} \cdots u_{n-1}}^{(n-1)} \prec \Psi^{2(n-1)+1}+\delta_{a b} \Psi^{2(n-1)} .
$$

In the following we will simplify the notation by setting $Y_{a b ; u_{1} \cdots u_{n}}^{(n)} \equiv Y_{a b}^{(n)}$. For any fixed integer $p \geqslant 1$ Lemma 7.1 implies that

$$
\begin{aligned}
\mathbb{E}\left|Y_{a b}^{(n)}\right|^{2 p} \prec & \left|\mathbb{E} \sum_{i_{2}, \ldots, i_{n}} \breve{\sigma}_{u_{1} i_{2}}^{(1)} \sigma_{u_{2} i_{2}}^{(2)} \cdots \sigma_{u_{n} i_{n}}^{(n)} G_{a i_{2}} G_{i_{2} i_{3}} \cdots G_{i_{n} b}\left(Y_{a b}^{(n)}\right)^{p-1}\left(\bar{Y}_{a b}^{(n)}\right)^{p}\right| \\
& +\mid \mathbb{E} \sum_{i_{1}, \ldots, i_{n+1}} \breve{\sigma}_{u_{1} i_{1}}^{(1)} \cdots \sigma_{u_{n} i_{n}}^{(n)} S_{i_{1} i_{n+1}} \\
& \times\left[G_{a i_{1}}\left(G_{i_{n+1} i_{n+1}}-\mathfrak{m}\right) G_{i_{1} i_{2}}+G_{a i_{n+1}}\left(G_{i_{1} i_{1}}-\mathfrak{m}\right) G_{i_{n+1} i_{2}}\right] G_{i_{2} i_{3}} \cdots G_{i_{n} b}\left(Y_{a b}^{(n)}\right)^{p-1}\left(\bar{Y}_{a b}^{(n)}\right)^{p} \mid \\
& +\left|\mathbb{E} \sum_{i_{1}, \ldots, i_{n+1}} \breve{\sigma}_{u_{1} i_{1}}^{(1)} \cdots \sigma_{u_{n} i_{n}}^{(n)} S_{i_{1} i_{n+1}} G_{a i_{n+1}} G_{i_{1} i_{2}}\left[\partial_{i_{1} i_{n+1}} G_{i_{2} i_{3}} \cdots G_{i_{n} b}\right]\left(Y_{a b}^{(n)}\right)^{p-1}\left(\bar{Y}_{a b}^{(n)}\right)^{p}\right| \\
& +\left|\mathbb{E} \sum_{i_{1}, \ldots, i_{n+1}} \breve{\sigma}_{u_{1} i_{1}}^{(1)} \cdots \sigma_{u_{n} i_{n}}^{(n)} S_{i_{1} i_{n+1}} G_{a i_{n+1}} G_{i_{1} i_{2}} G_{i_{2} i_{3}} \cdots G_{i_{n} b}\left[\partial_{i_{1} i_{n+1}}\left(Y_{a b}^{(n)}\right)^{p-1}\left(\bar{Y}_{a b}^{(n)}\right)^{p}\right]\right| \\
& =(\mathrm{A})+(\mathrm{B})+(\mathrm{C})+(\mathrm{D}) .
\end{aligned}
$$

Let us now deal with the terms (A), (B), (C) and (D). To simplify the notation we rename $\breve{\sigma}^{(1)}$ by $\sigma^{(1)}$.

(A) Let us define the tensor $\Xi_{a b}^{c}:=W \sigma_{a b}^{(1)} \sigma_{c b}^{(2)}$. It is immediate to verify that for any $c$ we have that $\Xi^{c} \in \mathbb{S}$. Therefore, by using the induction hypothesis (7.30) one has

$$
\begin{aligned}
(\mathrm{A}) & \prec W^{-1}\left|\mathbb{E} \sum_{i_{2}, \ldots, i_{n}} \Xi_{u_{1} i_{2}}^{u_{2}} \sigma_{u_{3} i_{3}}^{(3)} \cdots \sigma_{u_{n} i_{n}}^{(n)} G_{a i_{2}} G_{i_{2} i_{3}} \cdots G_{i_{n} b}\left(Y_{a b}^{(n)}\right)^{p-1}\left(\bar{Y}_{a b}^{(n)}\right)^{p}\right| \\
& \prec\left(\Psi^{2} \mathbb{E}\left|Y_{a b}^{(n-1)}\right|\left|Y_{a b}^{(n)}\right|^{2 p-1}\right) \prec\left(\Psi^{2 n+1}+\delta_{a b} \Psi^{2 n}\right) \mathbb{E}\left|Y_{a b}^{(n)}\right|^{2 p-1} .
\end{aligned}
$$

(B) From (7.30) we obtain

$$
\begin{aligned}
(\mathrm{B}) & =\left|\mathbb{E} \sum_{i_{1}, i_{n+1}} \sigma_{u_{1} i_{1}}^{(1)} S_{i_{1} i_{n+1}}\left[\left(G_{i_{n+1} i_{n+1}}-\mathfrak{m}\right) G_{a i_{1}} Y_{i_{1} b}^{(n-1)}+\left(G_{i_{1} i_{1}}-\mathfrak{m}\right) G_{a i_{n+1}} Y_{i_{n+1} b}^{(n-1)}\right]\left(Y_{a b}^{(n)}\right)^{p-1}\left(\bar{Y}_{a b}^{(n)}\right)^{p}\right| \\
& \prec \Psi^{2 n+1} \mathbb{E}\left|Y_{a b}^{(n)}\right|^{2 p-1} .
\end{aligned}
$$


(C) Using (7.30), one has

$$
\begin{aligned}
(\mathrm{C}) \leqslant & \left|\mathbb{E} \sum_{i_{1}, \ldots, i_{n+1}} \sigma_{u_{1} i_{1}}^{(1)} \cdots \sigma_{u_{n} i_{n}}^{(n)} S_{i_{1} i_{n+1}} G_{a i_{n+1}} G_{i_{1} i_{2}} \sum_{k=2}^{n-1} G_{i_{k} i_{1}} G_{i_{n+1} i_{k+1}}\left(\prod_{\substack{l=2 \\
l \neq k}}^{n-1} G_{i_{l} i_{l+1}}\right) G_{i_{n} b}\left(Y_{a b}^{(n)}\right)^{p-1}\left(\bar{Y}_{a b}^{(n)}\right)^{p}\right| \\
& +\left|\mathbb{E} \sum_{i_{1}, \ldots, i_{n+1}} \sigma_{u_{1} i_{1}}^{(1)} \cdots \sigma_{u_{n} i_{n}}^{(n)} S_{i_{1} i_{n+1}} G_{a i_{n+1}} G_{i_{1} i_{2}} \cdots G_{i_{n} i_{1}} G_{i_{n+1} b}\left(Y_{a b}^{(n)}\right)^{p-1}\left(\bar{Y}_{a b}^{(n)}\right)^{p}\right| \\
& \prec \sum_{k=1}^{n-1} \mathbb{E} \sum_{i_{1}}\left|\sigma_{u_{1} i_{1}}^{(1)}\right|\left|Y_{i_{1} i_{1}}^{(k)}\left\|Y_{a b}^{(n-k)}\right\| Y_{a b}^{(n)}\right|^{2 p-1} \prec\left(\Psi^{2 n+1}+\delta_{a b} \Psi^{2 n}\right) \mathbb{E}\left|Y_{a b}^{(n)}\right|^{2 p-1}
\end{aligned}
$$

(D) Note that

$$
\partial_{i_{1} i_{n+1}} Y_{a b}^{(n)}=-\sum_{k=0}^{n} Y_{a i_{1}}^{(k)} Y_{i_{n+1} b}^{(n-k)}, \quad \partial_{i_{1} i_{n+1}} \bar{Y}_{a b}^{(n)}=-\sum_{k=0}^{n} \bar{Y}_{a i_{n+1}}^{(k)} \bar{Y}_{i_{1} b}^{(n-k)}
$$

where $Y_{a b}^{(0)}:=G_{a b}$. Therefore we get for some $\xi \in \mathbb{S}$ (ignoring the complex conjugation which does not play any role here)

$$
\begin{aligned}
(\mathrm{D}) & \prec\left|\mathbb{E} \sum_{k=0}^{n} \sum_{i_{1}, i_{n+1}} \sigma_{u_{1} i_{1}}^{(1)} S_{i_{1} i_{n+1}} G_{a i_{n+1}} Y_{a i_{1}}^{(k)} Y_{i_{n+1} b}^{(n-k)} Y_{i_{1} b}^{(n-1)}\left(Y_{a b}^{(n)}\right)^{2 p-2}\right| \\
& \prec\left|\mathbb{E} \sum_{k=1}^{n-1} \sum_{i_{1}, i_{n+1}} \sigma_{u_{1} i_{1}}^{(1)} S_{i_{1} i_{n+1}} G_{a i_{n+1}} Y_{a i_{1}}^{(k)} Y_{i_{n+1} b}^{(n-k)} Y_{i_{1} b}^{(n-1)}\left(Y_{a b}^{(n)}\right)^{2 p-2}\right| \\
& +\left|\mathbb{E} \sum_{i_{1} i_{n+1}} \sigma_{u_{1}, i_{1}}^{(1)} S_{i_{1} i_{n+1}} G_{a i_{n+1}}\left[G_{a i_{1}} Y_{i_{n+1} b}^{(n)}+G_{i_{n+1} b} Y_{a i_{1}}^{(n)}\right] Y_{i_{1} b}^{(n-1)}\left(Y_{a b}^{(n)}\right)^{2 p-2}\right| \\
& \prec\left(\Psi^{4 n+2}+\delta_{a b} \Psi^{4 n}\right) \mathbb{E}\left|Y_{a b}^{(n)}\right|^{2 p-2}+(\mathrm{E})
\end{aligned}
$$

where

$$
(\mathrm{E})=\mathbb{E} \sum_{i_{1}, \ldots, i_{n+1}} \sigma_{u_{1} i_{1}}^{(1)} S_{i_{1} i_{n+1}} G_{a i_{n+1}}\left[G_{a i_{1}} Y_{i_{n+1} b}^{(n)}+G_{i_{n+1} b} Y_{a i_{1}}^{(n)}\right] Y_{i_{1} b}^{(n-1)}\left(Y_{a b}^{(n)}\right)^{2 p-2} .
$$

In order to bound (E) let suppose that $a \neq b$ and that

$$
Y_{a b}^{(n)} \prec \lambda^{(n)}, \quad \lambda^{(n)} \in\left[\Psi^{2 n+1}, L^{C}\right] .
$$

Note that from (7.30) we can easily get that $Y_{x y}^{(n)} \prec \Psi^{2 n}$ for any $x, y \in \mathbb{T}_{L}$, so from (7.33) we have

$$
\begin{aligned}
& (\mathrm{E}) \prec\left(\lambda^{(n)} \Psi^{2 n+1}+\Psi^{4 n+2}\right) \mathbb{E}\left|Y_{a b}^{(n)}\right|^{2 p-2}, \quad a \neq b \\
& (\mathrm{E}) \prec \Psi^{4 n} \mathbb{E}\left|Y_{a b}^{(n)}\right|^{2 p-2}, \quad a=b .
\end{aligned}
$$

Putting together the contribution form all the terms (A), (B), (C), (D), (E) and using Lemma 6.3, we can conclude that $Y_{a b}^{(n)} \prec \Psi^{2 n+1}+\delta_{a b} \Psi^{2 n}$.

7.2. Loop estimates: proof of (6.4). As for (6.3), we will proceed by induction on $n$ : from (7.12) we know that $Z_{a b}^{(2)} \prec \Psi^{4}+\xi_{a b}$ where $\xi \in \mathbb{S}$. As induction hypothesis, let us assume that

$$
Z_{a b ; u_{3} \cdots u_{n-1}}^{(n-1)} \prec \Psi^{2(n-1)}+\xi_{a b} \Psi^{2(n-3)}, \quad \xi \in \mathbb{S} .
$$

We need the following auxiliary lemma. Recall that $g^{b}=g-\mathbb{E} g$. 
Lemma 7.3. Let

$$
B_{a b}^{(n)}:=\sum_{i_{1}, \ldots, i_{n}, i_{n+1}} \sigma_{a i_{1}}^{(1)} \sigma_{b i_{2}}^{(2)} \cdots \sigma_{u_{n} i_{n}}^{(n)} S_{i_{1} i_{n+1}} G_{i_{1} i_{1}}^{b} G_{i_{n+1} i_{2}} G_{i_{n} i_{n+1}} G_{i_{2} i_{3}} \cdots G_{i_{n-1} i_{n}}
$$

with $\sigma^{(1)}, \sigma^{(2)}, \ldots, \sigma^{(n)} \in \mathbb{S}$. Then

$$
B_{a b}^{(n)} \prec \Psi^{2 n}
$$

Proof. See Section 7.3.

In the following we will adopt the simplified notation $Z_{a b}^{(n)} \equiv Z_{a b ; u_{3} \cdots u_{n}}^{(n)}$. As before, from Lemma 7.1 for any $p \geqslant 1$ we deduce that

$$
\begin{aligned}
& \mathbb{E}\left|Z_{a b}^{(n)}\right|^{2 p} \prec\left|\mathbb{E} \sum_{i_{2}, \ldots, i_{n}} \breve{\sigma}_{a i_{2}}^{(1)} \sigma_{b i_{2}}^{(2)} \cdots \sigma_{u_{n} i_{n}}^{(n)} G_{i_{2} i_{3}} \cdots G_{i_{n-1} i_{n}} G_{i_{n} i_{2}}\left(Z_{a b}^{(n)}\right)^{p-1}\left(\bar{Z}_{a b}^{(n)}\right)^{p}\right| \\
& +\left|\mathbb{E} \sum_{i_{1}, \ldots, i_{n+1}} \breve{\sigma}_{a i_{1}}^{(1)} \sigma_{b i_{2}}^{(2)} \cdots \sigma_{u_{n} i_{n}}^{(n)} S_{i_{1} i_{n+1}}\left(G_{i_{n+1} i_{n+1}}-\mathfrak{m}\right) G_{i_{n} i_{1}} G_{i_{1} i_{2}} G_{i_{2} i_{3}} \cdots G_{i_{n}-1} i_{n}\left(Z_{a b}^{(n)}\right)^{p-1}\left(\bar{Z}_{a b}^{(n)}\right)^{p}\right| \\
& +\left|\mathbb{E} \sum_{i_{1}, \ldots, i_{n+1}} \breve{\sigma}_{a i_{1}}^{(1)} \sigma_{b i_{2}}^{(2)} \cdots \sigma_{u_{n} i_{n}}^{(n)} S_{i_{1} i_{n+1}}\left(G_{i_{1} i_{1}}-\mathfrak{m}\right) G_{i_{n} i_{n+1}} G_{i_{n+1} i_{2}} G_{i_{2} i_{3}} \cdots G_{i_{n-1} i_{n}}\left(Z_{a b}^{(n)}\right)^{p-1}\left(\bar{Z}_{a b}^{(n)}\right)^{p}\right| \\
& +\left|\mathbb{E} \sum_{i_{1}, \ldots, i_{n+1}} \breve{\sigma}_{a i_{1}}^{(1)} \sigma_{b i_{2}}^{(2)} \cdots \sigma_{u_{n} i_{n}}^{(n)} S_{i_{1} i_{n+1}} G_{i_{n} i_{1}} G_{i_{n+1} i_{2}} \sum_{k=2}^{n-1} G_{i_{k} i_{n+1}} G_{i_{1} i_{k+1}}\left(\prod_{l=2}^{n-1} G_{i_{l} i_{l+1}}\right)\left(Z_{a b}^{(n)}\right)^{p-1}\left(\bar{Z}_{a b}^{(n)}\right)^{p}\right| \\
& +\left|\mathbb{E} \sum_{i_{1}, \ldots, i_{n+1}} \breve{\sigma}_{a i_{1}}^{(1)} \sigma_{b i_{2}}^{(2)} \cdots \sigma_{u_{n} i_{n}}^{(n)} S_{i_{1} i_{n+1}} G_{i_{n+1} i_{2}} G_{i_{2} i_{3}} \cdots G_{i_{n-1} i_{n}} G_{i_{n} i_{1}} \partial_{i_{n+1} i_{1}}\left(Z_{a b}^{(n)}\right)^{p-1}\left(\bar{Z}_{a b}^{(n)}\right)^{p}\right| \\
& =(\mathrm{A})+(\mathrm{B})+(\mathrm{C})+(\mathrm{D})+(\mathrm{E}) .
\end{aligned}
$$

In the following we simplify the notation by renaming $\breve{\sigma}^{(1)}$ as $\sigma^{(1)}$. Let us now look at the different terms.

(A) By using (6.3) and the properties of the matrix family $\mathbb{S}$, for some $\xi \in \mathbb{S}$ one easily gets that

$$
(\mathrm{A}) \prec \Psi^{2(n-2)} \sum_{i_{2}}\left|\sigma_{a i_{2}}^{(1)}\right|\left|\sigma_{b i_{2}}^{(2)}\right| \mathbb{E}\left|Z_{a b}^{(n)}\right|^{2 p-1} \prec \xi_{a b} \Psi^{2(n-2)} \mathbb{E}\left|Z_{a b}^{(n)}\right|^{2 p-1} .
$$

(B) We note that

$$
(\mathrm{B}) \prec\left|\mathbb{E} \sum_{i_{1}, i_{n+1}} \sigma_{a i_{1}}^{(1)} S_{i_{1} i_{n+1}}\left(G_{i_{n+1} i_{n+1}}-\mathfrak{m}\right) Y_{i_{1} i_{1}}^{(n-1)}\left(Z_{a b}^{(n)}\right)^{p-1}\left(\bar{Z}_{a b}^{(n)}\right)^{p}\right|
$$

thus from (6.5) and (6.3) we get $(\mathrm{B}) \prec \Psi^{2 n} \mathbb{E}\left|Z_{c d}^{(n)}\right|^{2 p-1}$.

(C) From Lemma 7.3 and the fact that $\mathbb{E} G_{k k}-\mathfrak{m} \prec \Psi^{2}$, one can conclude that $(\mathrm{C}) \prec \Psi^{2 n} \mathbb{E}\left|Z_{a b}^{(n)}\right|^{2 p-1}$.

(D) We note that

$$
\begin{aligned}
(\mathrm{D}) & \prec\left|\mathbb{E} \sum_{k=2}^{n-1} \sum_{i_{1}} \sigma_{a i_{1}}^{(1)} Z_{i_{1} b}^{(k)} Y_{i_{1} i_{1}}^{(n-k)}\left(Z_{a b}^{(n)}\right)^{p-1}\left(\bar{Z}_{a b}^{(n)}\right)^{p}\right| \prec\left(\Psi^{2 n}+\Psi^{2(n-2)} \sum_{i_{1}}\left|\sigma_{a i_{1}}^{(1)}\right| \xi_{i_{1} b}^{\prime}\right) \mathbb{E}\left|Z_{a b}^{(n)}\right|^{2 p-1} \\
& \prec\left(\Psi^{2 n}+\xi_{a b} \Psi^{2(n-2)}\right) \mathbb{E}\left|Z_{a b}^{(n)}\right|^{2 p-1}
\end{aligned}
$$

where we used (7.34) and (6.3). Here $\xi_{a b}=\sum_{i}\left|\sigma_{a i}^{(1)}\right| \xi_{i b}^{\prime}$ and $\xi, \xi^{\prime} \in \mathbb{S}$. 
(E) Note that

$$
\begin{aligned}
& \partial_{i_{n+1} i_{1}} Z_{a b}^{(n)}=-\left.\sum_{k=1}^{n} \sum_{j_{1}, \ldots, j_{n}} \sigma_{a j_{1}}^{(1)} \sigma_{b j_{2}}^{(2)} \cdots \sigma_{u_{n} j_{n}}^{(n)} G_{j_{k} i_{n+1}} G_{i_{1} j_{k+1}} \prod_{l=1, l \neq k}^{n} G_{j_{l} j_{l+1}}\right|_{j_{n+1}=j_{1}} \\
& \partial_{i_{n+1} i_{1}} \bar{Z}_{a b}^{(n)}=-\left.\sum_{k=1}^{n} \sum_{j_{1}, \ldots, j_{n}} \sigma_{a j_{1}}^{(1)} \sigma_{b j_{2}}^{(2)} \cdots \sigma_{u_{n} j_{n}}^{(n)} \bar{G}_{j_{k} i_{1}} \bar{G}_{i_{n+1} j_{k+1}} \prod_{l=1, l \neq k}^{n} \bar{G}_{j_{l} j_{l+1}}\right|_{j_{n+1}=j_{1}},
\end{aligned}
$$

thus from (6.3) we get

$$
(\mathrm{E}) \prec \mathbb{E} \sum_{i_{i}, i_{n+1}}\left|\sigma_{a i_{1}}^{(1)}\right| S_{i_{1} i_{n+1}}\left|Y_{i_{1} i_{n+1}}^{(n)}\right|\left|Y_{i_{i} i_{n+1}}^{(n-1)}\right|\left|Z_{a b}^{(n)}\right|^{2 p-2} \prec \Psi^{4 n} \mathbb{E}\left|Z_{a b}^{(n)}\right|^{2 p-2} .
$$

Collecting all the terms and applying Lemma 6.3 we get the claim.

7.3. Proof of Lemma 7.3. From (7.20) we know that $B_{a b}^{(2)} \prec \Psi^{4}$. We want to show that $B_{a b}^{(n)} \prec \Psi^{2 n}$ and we will proceed by induction by setting the hypothesis

$$
B_{a b}^{(n-1)} \prec \Psi^{2(n-1)} .
$$

For any fixed integer $p \geqslant 1$ we consider the moment $\mathbb{E}\left|B_{a b}\right|^{2 p}$ and by invoking Lemma 7.1, we get

$$
\mathbb{E}\left|B_{a b}^{(n)}\right|^{2 p} \prec(\mathrm{A})+(\mathrm{B})+(\mathrm{C})+(\mathrm{D})+(\mathrm{E})+(\mathrm{F})
$$

where the explicit form of (A), (B), (C), (D), (E) and (F) is given below. We proceed term by term.

(A) By using the induction hypothesis (7.36) and the properties of the matrices in $\mathbb{S}$ one has

$$
\begin{aligned}
(\mathrm{A})= & \mid \mathbb{E} \sum_{i_{1}, \ldots, i_{n-1}, i_{n+1}} \sigma_{a i_{1}}^{(1)} \sigma_{b i_{2}}^{(2)} \cdots \sigma_{u_{n-1} i_{n-1}}^{(n-1)} \breve{\sigma}_{u_{n} i_{n-1}}^{(n)} S_{i_{1} i_{n+1}} \\
& \times G_{i_{1} i_{1}}^{b} G_{i_{n-1} i_{n+1}} G_{i_{n+1} i_{2}} G_{i_{2} i_{3}} \cdots G_{i_{n-2} i_{n-1}}\left(B_{a b}^{(n)}\right)^{p-1}\left(\bar{B}_{a b}^{(n)}\right)^{p} \mid \\
& \prec W^{-1} \Psi^{2(n-1)} \mathbb{E}\left|B_{a b}^{(n)}\right|^{2 p-1} \prec \Psi^{2 n} \mathbb{E}\left|B_{a b}^{(n)}\right|^{2 p-1} .
\end{aligned}
$$

(B) From (6.3) we have

$$
\begin{aligned}
(\mathrm{B})= & \mid \mathbb{E} \sum_{i_{1}, \ldots, i_{n}, i_{n+1}, i_{n+2}} \sigma_{a i_{1}}^{(1)} \sigma_{b i_{2}}^{(2)} \cdots \sigma_{u_{n-1} i_{n-1}}^{(n-1)} \breve{\sigma}_{u_{n} i_{n}}^{(n)} S_{i_{1} i_{n+1}} S_{i_{n-1} i_{n+2}} \\
& \times G_{i_{1} i_{1}}^{b} G_{i_{n} i_{n+1}} G_{i_{n+1} i_{2}} G_{i_{2} i_{3}} \cdots G_{i_{n-3} i_{n-2}} G_{i_{n-2} i_{n-1}} G_{i_{n-1} i_{n}}\left(G_{i_{n+2} i_{n+2}}-\mathfrak{m}\right)\left(B_{a b}^{(n)}\right)^{p-1}\left(\bar{B}_{a b}^{(n)}\right)^{p} \mid \\
\prec & \mathbb{E} \sum_{i_{1} i_{n-1} i_{n+2}}\left|\sigma_{a i_{1}}^{(1)} \sigma_{u_{n-1} i_{n-1}}^{(n-1)} S_{i_{n-1} i_{n+2}} G_{i_{1} i_{1}}^{b}\left(G_{i_{n+2} i_{n+2}}-\mathfrak{m}\right) Y_{i_{n-1} i_{n-1}}^{(n-1)}\right|\left|B_{a b}^{(n)}\right|^{2 p-1} \prec \Psi^{2 n} \mathbb{E}\left|B_{a b}^{(n)}\right|^{2 p-1} .
\end{aligned}
$$

(C) Similarly from (6.3) we have

$$
\begin{aligned}
(\mathrm{C})= & \mid \mathbb{E} \sum_{i_{1}, \ldots, i_{n+2}} \sigma_{a i_{1}}^{(1)} \sigma_{b i_{2}}^{(2)} \cdots \sigma_{u_{n-1} i_{n-1}}^{(n-1)} \breve{\sigma}_{u_{n} i_{n}}^{(n)} S_{i_{1} i_{n+1}} S_{i_{n-1} i_{n+2}} \\
& \times G_{i_{1} i_{1}}^{b} G_{i_{n} i_{n+1}} G_{i_{n+1} i_{2}} G_{i_{2} i_{3}} \cdots G_{i_{n-3} i_{n-2}} G_{i_{n-2} i_{n+2}} G_{i_{n+2} i_{n}}\left(G_{i_{n-1} i_{n-1}}-\mathfrak{m}\right)\left(B_{a b}^{(n)}\right)^{p-1}\left(\bar{B}_{a b}^{(n)}\right)^{p} \mid \\
& \prec \mathbb{E} \sum_{i_{1}, i_{n-1}, i_{n+2}}\left|\sigma_{a i_{1}}^{(1)} \sigma_{u_{n-1} i_{n-1}}^{(n-1)} S_{i_{n-1} i_{n+2}} G_{i_{1} i_{1}}^{b}\left(G_{i_{n-1} i_{n-1}}-\mathfrak{m}\right) Y_{i_{n+2} i_{n+2}}^{(n-1)}\right|\left|B_{a b}^{(n)}\right|^{2 p-1} \prec \Psi^{2 n} \mathbb{E}\left|B_{a b}^{(n)}\right|^{2 p-1} .
\end{aligned}
$$


(D) By (6.3) one has

$$
\begin{aligned}
(\mathrm{D})= & \mid \mathbb{E} \sum_{i_{1}, \ldots, i_{n+2}} \sigma_{a i_{1}}^{(1)} \sigma_{b i_{2}}^{(2)} \cdots \sigma_{u_{n-1} i_{n-1}}^{(n-1)} \breve{\sigma}_{u_{n} i_{n}}^{(n)} S_{i_{1} i_{n+1}} S_{i_{n-1} i_{n+2}} \\
& \times G_{i_{1} i_{n+2}} G_{i_{n+2} i_{n}} G_{i_{n} i_{n+1}} G_{i_{n+1} i_{2}} G_{i_{2} i_{3}} \cdots G_{i_{n-3} i_{n-2}} G_{i_{n-2} i_{n-1}} G_{i_{n-1} i_{1}}\left(B_{a b}^{(n)}\right)^{p-1}\left(\bar{B}_{a b}^{(n)}\right)^{p} \mid \\
& \prec \mathbb{E} \sum_{i_{1}, i_{n+2}, i_{n-1}}\left|\sigma_{a i_{1}}^{(1)} \sigma_{u_{n-1} i_{n-1}}^{(n-1)} S_{i_{n-1} i_{n+2}} G_{i_{1} i_{n+2}} G_{i_{n-1} i_{1}} Y_{i_{n+2} i_{n-1}}^{(n-1)}\left(B_{a b}^{(n)}\right)^{p-1}\left(\bar{B}_{a b}^{(n)}\right)^{p}\right| \prec \Psi^{2 n} \mathbb{E}\left|B_{a b}^{(n)}\right|^{2 p-1} .
\end{aligned}
$$

(E) Again using (6.3), one gets

$$
\begin{aligned}
(\mathrm{E})= & \mid \mathbb{E} \sum_{i_{1}, \ldots, i_{n+2}} \sigma_{a i_{1}}^{(1)} \sigma_{b i_{2}}^{(2)} \cdots \sigma_{u_{n-1} i_{n-1}}^{(n-1)} \breve{\sigma}_{u_{n} i_{n}}^{(n)} S_{i_{1} i_{n+1}} S_{i_{n-1} i_{n+2}} \\
& \times G_{i_{1} i_{1}}^{b} G_{i_{n+2} i_{n}} G_{i_{n-2} i_{n-1}}\left[\left.\sum_{k=0}^{n-3} G_{i_{k} i_{n+2}} G_{i_{n-1} i_{k+1}}\left(\prod_{\substack{l=0 \\
l \neq k}}^{n-3} G_{i_{l} i_{l+1}}\right)\right|_{i_{0}=i_{n}}\right]\left(B_{a b}^{(n)}\right)^{p-1}\left(\bar{B}_{a b}^{(n)}\right)^{p} \mid \\
& \prec \mathbb{E} \sum_{k=0}^{n-3} \sum_{i_{n-1}}\left|\sigma_{u_{n-1} i_{n-1}}^{(n-1)} B_{a b}^{(k+2)} Y_{i_{n-1} i_{n-1}}^{(n-2-k)} \| B_{a b}^{(n)}\right|^{2 p-1} \prec \Psi^{2 n} \mathbb{E}\left|B_{a b}^{(n)}\right|^{2 p-1} .
\end{aligned}
$$

(F) We note that

$$
\begin{aligned}
& \partial_{i_{n+2} i_{n-1}} B_{a b}^{(n)}=\sum_{j_{1}, \ldots, j_{n}, j_{n+1}} \sigma_{a i_{1}}^{(1)} \sigma_{b i_{2}}^{(2)} \cdots \sigma_{u_{n} i_{n}}^{(n)} S_{j_{1} j_{n+1}} \\
& \times\left[G_{j_{1} i_{n+2}} G_{i_{n-1} j_{1}}\left(\left.\prod_{l=2}^{n+1} G_{j_{l} j_{l+1}}\right|_{j_{n+2}=j_{2}}\right)+G_{j_{1} j_{1}}^{b} \sum_{k=2}^{n+1} G_{j_{k} i_{n+2}} G_{i_{n-1} j_{k+1}}\left(\left.\prod_{l=2, l \neq k}^{n+1} G_{j_{l} j_{l+1}}\right|_{j_{n+2}=j_{2}}\right)\right] \\
& \partial_{i_{n+2} i_{n-1}} \bar{B}_{a b}^{(n)}=\sum_{j_{1}, \ldots, j_{n}, j_{n+1}} \sigma_{a i_{1}}^{(1)} \sigma_{b i_{2}}^{(2)} \cdots \sigma_{u_{n} i_{n}}^{(n)} S_{j_{1} j_{n+1}} \\
& \times\left[\bar{G}_{j_{1} i_{n-1}} \bar{G}_{i_{n+2} j_{1}}\left(\left.\prod_{l=2}^{n+1} \bar{G}_{j_{l} j_{l+1}}\right|_{j_{n+2}=j_{2}}\right)+\bar{G}_{j_{1} j_{1}}^{b} \sum_{k=2}^{n+1} \bar{G}_{j_{k} i_{n-1}} \bar{G}_{i_{n+2} j_{k+1}}\left(\left.\prod_{l=2, l \neq k}^{n+1} \bar{G}_{j_{l} j_{l+1}}\right|_{j_{n+2}=j_{2}}\right)\right],
\end{aligned}
$$

thus from (7.37) and (7.38) and by using the trivial bound $Z_{a b}^{(n)} \prec \Psi^{2(n-1)}$ we get

$$
\begin{aligned}
(\mathrm{F})= & \mid \mathbb{E} \sum_{i_{1}, \ldots, i_{n+2}} \sigma_{a i_{1}}^{(1)} \sigma_{b i_{2}}^{(2)} \cdots \breve{\sigma}_{u_{n} i_{n}}^{(n)} S_{i_{1} i_{n+1}} S_{i_{n+2} i_{n-1}} \\
& \times G_{i_{1} i_{1}}^{b} G_{i_{n+2} i_{n}} G_{i_{n} i_{n+1}} G_{i_{n+1} i_{2}} G_{i_{2} i_{3}} \cdots G_{i_{n-2} i_{n-1}} \partial_{i_{n+2} i_{n-1}}\left(B_{a b}^{(n)}\right)^{p-1}\left(\bar{B}_{a b}^{(n)}\right)^{p} \mid \\
\prec & \mathbb{E} \sum_{i_{1}, j_{1}, i_{n-1}, i_{n+2}}\left|\sigma_{a i_{1}}^{(1)} \sigma_{a j_{1}}^{(1)} \sigma_{u_{n-1} i_{n-1}}^{(n-1)} S_{i_{n+2} i_{n-1}} G_{i_{1} i_{1}}^{b}\right| \\
& \times\left(\left|G_{j_{1} i_{n+2}} G_{i_{n-1} j_{1}}\right|+\left|G_{j_{1} i_{n-1}} G_{i_{n+2}} j_{1}\right|\right)\left|Y_{i_{n+2} i_{n-1}}^{(n-1)} Z_{j_{1} b}^{(n)}\right|\left|B_{a b}^{(n)}\right|^{2 p-2} \\
& +\mathbb{E} \sum_{i_{1}, j_{1}, i_{n-1}, i_{n+2}}\left|\sigma_{a i_{1}}^{(1)} \sigma_{a j_{1}}^{(1)} \sigma_{u_{n-1} i_{n-1}}^{(n-1)} S_{i_{n+2} i_{n-1}} G_{i_{1} i_{1}}^{b} G_{j_{1} j_{1}}^{b} Y_{i_{n+2} i_{n-1}}^{(n-1)} Y_{i_{n+2} i_{n-1}}^{(n)}\right|\left|B_{a b}^{(n)}\right|^{2 p-2} \\
& \prec \Psi^{4 n} \mathbb{E}\left|B_{a b}^{(n)}\right|^{2 p-2} .
\end{aligned}
$$

In conclusion, we obtained $\mathbb{E}\left|B_{a b}^{(n)}\right|^{2 p} \prec \Psi^{2 n} \mathbb{E}\left|B_{a b}^{(n)}\right|^{2 p-1}+\Psi^{4 n} \mathbb{E}\left|B_{a b}^{(n)}\right|^{2 p-2}$ which implies the desired estimate thanks to Lemma 6.3. 
7.4. Proof of (6.5). Since from (7.8) we have that $\mathbb{E} \sum_{a} \sigma_{i a}\left(G_{a a}-\mathfrak{m}\right) \prec \Psi^{2}$, to prove the claim we have to estimate

$$
X_{i}^{\prime}=\sum_{a} \sigma_{i a} G_{a a}^{b}
$$

We proceed as in the proof of Lemma 7.1: consider $z \mathbb{E}\left|X_{i}^{\prime}\right|^{2 p}=\mathbb{E} \sum_{a} \sigma_{i a} z G_{a a}^{b}\left(X_{i}^{\prime}\right)^{p-1}\left(\bar{X}_{i}^{\prime}\right)^{p}$. The cumulant expansion yields

$$
\begin{aligned}
& z \mathbb{E}\left|X_{i}^{\prime}\right|^{2 p}=-\mathbb{E} \sum_{a, b} \sigma_{i a} S_{a b}\left(G_{a a} G_{b b}\right)^{b}\left(X_{i}^{\prime}\right)^{p-1}\left(\bar{X}_{i}^{\prime}\right)^{p}+\mathbb{E} \sum_{a, b} \sigma_{i a} S_{a b} G_{b a} \partial_{b a}\left(X_{i}^{\prime}\right)^{p-1}\left(\bar{X}_{i}^{\prime}\right)^{p} \\
&=- \mathbb{E} \sum_{a, b} \sigma_{i a} S_{a b}\left[G_{a a}^{b} G_{b b}^{b}-\mathbb{E} G_{a a}^{b} G_{b b}^{b}+G_{a a}^{b} \mathbb{E} G_{b b}+G_{b b}^{b} \mathbb{E} G_{a a}\right]\left(X_{i}^{\prime}\right)^{p-1}\left(\bar{X}_{i}^{\prime}\right)^{p} \\
&+\mathbb{E} \sum_{a, b} \sigma_{i a} S_{a b} G_{b a} \partial_{b a}\left(X_{i}^{\prime}\right)^{p-1}\left(\bar{X}_{i}^{\prime}\right)^{p} \\
&=-\mathfrak{m} \mathbb{E}\left|X_{i}^{\prime}\right|^{2 p}-\mathfrak{m} \mathbb{E} \sum_{a, b} \sigma_{i a} S_{a b} G_{b b}^{b}\left(X_{i}^{\prime}\right)^{p-1}\left(\bar{X}_{i}^{\prime}\right)^{p}+\mathbb{E} \sum_{a, b} \sigma_{i a} S_{a b} G_{b a} \partial_{b a}\left(X_{i}^{\prime}\right)^{p-1}\left(\bar{X}_{i}^{\prime}\right)^{p} \\
&-\mathbb{E} \sum_{a, b} \sigma_{i a} S_{a b}\left[G_{a a}^{b} G_{b b}^{b}-\mathbb{E} G_{a a}^{b} G_{b b}^{b}+G_{a a}^{b}\left(\mathbb{E} G_{b b}-\mathfrak{m}\right)+G_{b b}^{b}\left(\mathbb{E} G_{a a}-\mathfrak{m}\right)\right]\left(X_{i}^{\prime}\right)^{p-1}\left(\bar{X}_{i}^{\prime}\right)^{p} .
\end{aligned}
$$

where we used that

$$
(f g)^{b}=f^{b} g^{b}-\mathbb{E} f b g^{b}+f^{b} \mathbb{E} g+g^{b} \mathbb{E} f .
$$

Let $\tilde{X}_{i}:=\sum_{a} S_{i a} G_{a a}^{b}$. Expanding $\mathbb{E}\left|\tilde{X}_{i}\right|^{2 p}$ as we did for $\mathbb{E}\left|X_{i}^{\prime}\right|^{2 p}$ and using the properties of the matrix $\mathcal{L}$ defined in (7.5), we get

$$
\begin{aligned}
\mathbb{E}\left|\tilde{X}_{i}\right|^{2 p}=\mathbb{E} & \sum_{a, b} \tau_{i a} S_{a b} G_{b a} \partial_{b a}\left(X_{i}^{\prime}\right)^{p-1}\left(\bar{X}_{i}^{\prime}\right)^{p} \\
& -\mathbb{E} \sum_{a, b} \tau_{i a} S_{a b}\left[G_{a a}^{b} G_{b b}^{b}-\mathbb{E} G_{a a}^{b} G_{b b}^{b}+G_{a a}^{b}\left(\mathbb{E} G_{b b}-\mathfrak{m}\right)+G_{b b}^{b}\left(\mathbb{E} G_{a a}-\mathfrak{m}\right)\right]\left(X_{i}^{\prime}\right)^{p-1}\left(\bar{X}_{i}^{\prime}\right)^{p},
\end{aligned}
$$

where $\tau=\mathcal{L} S$. Thus, from (7.40) and (7.39) we get

$$
\begin{aligned}
\mathbb{E}\left|X_{i}^{\prime}\right|^{2 p} & \prec\left|\mathbb{E} \sum_{a, b} \breve{\sigma}_{i a} S_{a b} G_{b a} \partial_{b a}\left(X_{i}^{\prime}\right)^{p-1}\left(\bar{X}_{i}^{\prime}\right)^{p}\right| \\
& +\left|\mathbb{E} \sum_{a, b} \breve{\sigma}_{i a} S_{a b}\left[G_{a a}^{b} G_{b b}^{b}-\mathbb{E} G_{a a}^{b} G_{b b}^{b}+G_{a a}^{b}\left(\mathbb{E} G_{b b}-\mathfrak{m}\right)+G_{b b}^{b}\left(\mathbb{E} G_{a a}-\mathfrak{m}\right)\right]\left(X_{i}^{\prime}\right)^{p-1}\left(\bar{X}_{i}^{\prime}\right)^{p}\right| \\
& \prec\left|\mathbb{E} \sum_{a, b} \breve{\sigma}_{i a} S_{a b} G_{b a} \partial_{b a}\left(X_{i}^{\prime}\right)^{p-1}\left(\overline{X_{i}^{\prime}}\right)^{p}\right|+\Psi^{2} \mathbb{E}\left|X_{i}^{\prime}\right|^{2 p}
\end{aligned}
$$

where in the last line we used $\mathbb{E}\left(G_{i i}-\mathfrak{m}\right) \prec \Psi$ in (7.10) and $G_{i i}^{b} \prec \Psi$. To finish the proof, we compute the derivative

$$
\partial_{a b} X_{i}^{\prime}=-\sum_{c} \sigma_{i c} G_{c a} G_{b c} \prec \Psi^{3}+\delta_{a b} \Psi^{2}
$$

where we used (7.11). Therefore, (7.41) becomes

$$
\mathbb{E}\left|\tilde{X}_{i}\right|^{2 p} \prec \Psi^{2} \mathbb{E}\left|X_{i}^{\prime}\right|^{2 p-1}+\Psi^{4} \mathbb{E}\left|X_{i}^{\prime}\right|^{2 p-2} .
$$

By invoking Lemma 6.3, we finally get $X_{i}^{\prime} \prec \Psi^{2}$. The proof of the second estimate in (6.5), i.e. $\sum_{k} \sigma_{i k} G_{k j} \prec \Psi^{2}$, is completely analogous to the one just presented for $X_{i} \prec \Psi^{2}$ and it is omitted. 


\section{Extension to non-Gaussian distribution and general complex case}

In this section we explain how to deal with the more general case when $H_{i j}$ is not necessarily Gaussian distributed and when $\mathbb{E} H_{i j}^{2} \neq 0$.

Let us start with the non Gaussian corrections: we point out that the way we are going to control them is insensitive to whether $\mathbb{E} H_{i j}^{2}=0$ or not, therefore, for the sake of simplicity, we will assume that $\mathbb{E} H_{i j}^{2}=0$. We recall that the Gaussian case is easier because, adopting the notation of Lemma 3.3, the cumulants of order $p+q=k \geqslant 3$ vanish, which implies that the cumulant expansion formula (3.1) is truncated at $\ell=1$ with $R_{2}=0$. When $H_{i j}$ is not Gaussian we have to estimate all the higher order terms in (3.1).

We will show explicitly that the non Gaussian terms do not modify the bounds that we got earlier in (5.8), (7.11) and (7.12) since they are at most of the same magnitude as the Gaussian terms. Heuristically, the reason is that, as stated in Lemma 3.3, we have

$$
\mathcal{C}^{(p, q)}\left(H_{i j}\right)=O\left(S_{i j}^{k / 2}\right) \prec S_{i j} \Psi^{k-2}, \quad k=p+q .
$$

Throughout this whole Section 8 we will assume that $\Psi$ is an admissible control parameter such that the a prior bound $\Lambda^{2} \prec \Psi^{2}$ holds true.

Note that (8.1) implies that in the higher order contributions of the full cumulant expansion (3.1) the huge number of resolvent entries produced by the derivatives is compensated by the smallness of the high order cumulants.

In particular, the strategy is to expand these derivatives via the Leibniz rule (see (8.4), (8.12) and (8.22) below): only few terms in this expansion need to be treated carefully (see for example (8.8), (8.9), (8.13) and (8.26)), while all the others are easily bounded by using (8.1), the Ward identity and $\Lambda^{2} \prec \Psi^{2}$.

Moreover, for (7.11) and (7.12) we can still employ the same self-consistent scheme that we set up in Lemma 7.1 in the non Gaussian case: the only modification is that the non Gaussian terms will appear just as additional contributions on the right hand side of (7.1).

The same method can be applied to prove that all the other results in Proposition 5.1 and lemmata 6.1, 7.2 and 7.3 remain valid when $H$ is non Gaussian. Finally, we will briefly discuss also how to control the additional terms arising in the cumulant expansion when $\mathbb{E} H_{i j}^{2} \neq 0$.

8.1. Non Gaussian terms in (5.8). In this section we explain the proof of (5.8) when the entries of $H$ are not Gaussian. Suppose there is some $\lambda \in\left[L^{-1}, L\right]$ such that $\mathcal{Q}_{x y} \prec \lambda$ for all $x, y \in \mathbb{T}_{L}$. As in Section 6, we would like to have a bound on $\mathbb{E}\left|Q_{y y}\right|^{2 p}$. By looking at the proof in Section 6 carefully, we see that we used two cumulant expansions in the proof: the first one is in (6.9) and the second one is in (6.20). We now want to control the additional non Gaussian terms arising from them.

Let us look at the estimate of (6.20). Starting from the LHS of (6.20), we need to consider the additional terms

$$
\begin{aligned}
& \left(\frac{1}{\sqrt{L}}\right)^{n} \sum_{\substack{w, t \geqslant 0 \\
w+t=2}}^{K} \frac{1}{w ! t !} \mathcal{C}^{(w, t+1)}\left(H_{j_{n+1} i_{n+1}}\right) \mathbb{E} \sum_{i_{1}, j_{1}, \ldots, i_{n+1}, j_{n+1}} \sigma_{i_{1} j_{1}}^{(1)} \cdots \sigma_{i_{n} j_{n}}^{(n)} \\
& \partial_{j_{n+1} i_{n+1}}^{w} \partial_{i_{n+1} j_{n+1}}^{t}\left(G_{j_{1} y} \bar{G}_{i_{1} y} V_{1,2} \cdots V_{n-1, n} S_{\mathbf{v} i_{n+1}} G_{j_{n+1} j_{n}} \bar{G}_{i_{n+1} y} G_{i_{n} y} \cdot \mathcal{Q}_{y y}^{\alpha} \overline{\mathcal{Q}}_{y y}^{\beta}\right)+\sum_{j_{n+1}, i_{n+1}} R_{K+1}^{j_{n+1} i_{n+1}},
\end{aligned}
$$

where $R_{K+1}^{j_{n+1} i_{n+1}}$ is the remainder term defined similarly to $R_{K+1}$ in (3.2). Following a routine verification (one may refer to the proof of Lemma 4.6(i) in [13]), we see that for any $D>0$, there is $K=K(D) \in \mathbb{N}$ such that

$$
\sum_{j_{n+1}, i_{n+1}} R_{K+1}^{j_{n+1} i_{n+1}}=O\left(L^{-D}\right) .
$$

Now we are left with the estimate of the first sum in (8.2). Let us fix $(w, t) \in \mathbb{N} \times \mathbb{N}$ with $w+t=k \geqslant 2$. W.L.O.G we assume $w=k$ and $t=0$, and the general cases of $w, t$ follow in a similar fashion. Let us define

$$
\sigma_{j_{n+1} i_{n+1}}^{(n+1)}=\sqrt{L} W^{(k-1) / 2} S_{\mathbf{v} i_{n+1}} \mathcal{C}^{(k, 1)}\left(H_{j_{n+1} i_{n+1}}\right),
$$

which belongs to $\mathbb{S}$ thanks to (8.1). 
We would like to bound

$\frac{1}{L^{(n+1) / 2} W^{(k-1) / 2}} \mathbb{E} \sum_{i_{1}, j_{1}, \ldots, i_{n+1}, j_{n+1}} \sigma_{i_{1} j_{1}}^{(1)} \cdots \sigma_{i_{n+1} j_{n+1}}^{(n+1)} \partial_{j_{n+1} i_{n+1}}^{k}\left(G_{j_{1} y} \bar{G}_{i_{1} y} V_{1,2} \cdots V_{n-1, n} G_{j_{n+1} j_{n}} \bar{G}_{i_{n+1} y} G_{i_{n} y} \cdot \mathcal{Q}_{y y}^{\alpha} \overline{\mathcal{Q}}_{y y}^{\beta}\right)$.

By Leibniz's rule, we look at (8.3) by considering

$$
\begin{array}{r}
\frac{1}{L^{(n+1) / 2} W^{(k-1) / 2}} \mathbb{E} \sum_{i_{1}, j_{1}, \ldots, i_{n+1}, j_{n+1}} \sigma_{i_{1} j_{1}}^{(1)} \cdots \sigma_{i_{n+1} j_{n+1}}^{(n+1)}\left[\partial_{j_{n+1} i_{n+1}}^{s}\left(G_{j_{1} y} \bar{G}_{i_{1} y} V_{1,2} \cdots V_{n-1, n} G_{j_{n+1} j_{n}} \bar{G}_{i_{n+1} y} G_{i_{n} y}\right)\right] \\
\cdot\left(\partial_{j_{n+1} i_{n+1}}^{t_{1}} \mathcal{Q}_{y y} \cdots \partial_{j_{n+1} i_{n+1}}^{t_{\ell}} \mathcal{Q}_{y y}\right) \cdot Q_{y y}^{\alpha-\ell} \overline{\mathcal{Q}}_{y y}^{\beta},
\end{array}
$$

where $s+t_{1}+\cdots+t_{\ell}=k \geqslant 2$. Here we consider the case where the differential $\partial_{j_{n+1} i_{n+1}}$ is only applied to $Q_{y y}$, and the general case when $\partial_{j_{n+1} i_{n+1}}$ is also applied to $\bar{Q}_{y y}$ can be estimated in the similar fashion. Thus a bound on (8.4) implies the same bound on (8.3).

Case 1. Suppose $\ell \geqslant 2$. Let $\tilde{\mathcal{T}}$ be the collection of factors $G$ and $\bar{G}$ in (8.4) such that at least one of the two indices belongs to the class $\left\{i_{1}, j_{1}, \ldots, i_{n}, j_{n}\right\}$, and we have $|\tilde{\mathcal{T}}|=3 n+1$. As in Section 5.1, we can use Lemma 6.1(i) to show

$$
\left(\frac{1}{\sqrt{L}}\right)^{n} \sum_{i_{1}, j_{1}, \ldots, i_{n}, j_{n}} \sigma_{i_{1} j_{1}}^{(1)} \sigma_{i_{n} j_{n}}^{(n)} \prod_{t \in \tilde{\mathcal{T}}} t \prec\left(\frac{\Psi^{3}}{\sqrt{\eta}}+\frac{\Psi}{\sqrt{L \eta}}\right)^{n} \cdot \Psi
$$

By

$$
\partial_{j i} \mathcal{Q}_{y y}=-\mathcal{Q}_{j y} G_{i y}-\mathcal{Q}_{y i} \bar{G}_{j y}+S_{\mathbf{v} j} G_{i y} \bar{G}_{j y}-\sum_{k, l} S_{\mathbf{v} k} S_{k l} G_{l j} G_{i l}\left|G_{k y}\right|^{2}-\sum_{k, l} S_{\mathbf{v} k} S_{k l} \bar{G}_{k i} \bar{G}_{j k}\left|G_{l y}\right|^{2}
$$

and

$$
\partial_{j i} \overline{\mathcal{Q}}_{y y}=-\overline{\mathcal{Q}}_{i y} \bar{G}_{j y}-\overline{\mathcal{Q}}_{y j} G_{i y}+\bar{S}_{\mathbf{v} j} G_{i y} \bar{G}_{j y}-\sum_{k, l} \bar{S}_{\mathbf{v} l} S_{l k} G_{l j} G_{i l}\left|G_{k y}\right|^{2}-\sum_{k, l} \bar{S}_{\mathbf{v} l} S_{l k} \bar{G}_{k i} \bar{G}_{j k}\left|G_{l y}\right|^{2}
$$

together with Ward identity, we see that for any fixed $m \geqslant 1$,

$$
\partial_{j_{n+1} i_{n+1}}^{m} \mathcal{Q}_{y y} \prec \lambda+\frac{1}{\sqrt{L}}+\frac{\Psi^{3}}{\sqrt{\eta}} .
$$

By using the above bound for $\ell-2$ many factors in (8.4), together with (8.5) we have for some $p_{n+1} \in\left\{i_{n+1}, j_{n+1}\right\}$ with

$$
\begin{aligned}
(8.4) \prec & \frac{1}{L^{1 / 2} W^{(k-1) / 2}} \cdot\left(\frac{\Psi^{3}}{\sqrt{\eta}}+\frac{\Psi}{\sqrt{L \eta}}\right)^{n} \cdot \Psi \cdot\left(\lambda+\frac{1}{\sqrt{L}}+\frac{\Psi^{3}}{\sqrt{\eta}}\right)^{\ell-2} \\
& \cdot \mathbb{E} \mid \sum_{i_{n+1}, j_{n+1}} \sigma_{i_{n+1} j_{n+1}}^{(n+1)} \bar{G}_{p_{n+1} y} \partial_{j_{n+1} t_{n+1}}^{t_{1}} \mathcal{Q}_{y y} \partial_{j_{n+1} t_{2} t_{n+1}}^{\left.t_{y y}|\cdot| \mathcal{Q}_{y y}\right|^{\alpha+\beta-\ell}} \\
\prec & \frac{1}{L^{1 / 2} W^{(k-\ell+1) / 2}} \cdot\left(\frac{\Psi^{3}}{\sqrt{\eta}}+\frac{\Psi}{\sqrt{L \eta}}\right)^{n} \cdot \Psi \cdot\left(\lambda \Psi+\frac{\Psi}{\sqrt{L}}+\frac{\Psi^{3}}{\sqrt{\eta}}\right)^{\ell-2} \\
& \cdot \mathbb{E}\left|\sum_{i_{n+1}, j_{n+1}} \sigma_{i_{n+1} j_{n+1}}^{(n+1)} \bar{G}_{p_{n+1} y} \partial_{j_{n+1} i_{n+1}}^{t_{1}} \mathcal{Q}_{y y} \partial_{j_{n+1} t_{2} t_{n+1}}^{t_{y y}}\right| \cdot\left|\mathcal{Q}_{y y}\right|^{\alpha+\beta-\ell} .
\end{aligned}
$$

For $\max \left\{t_{1}, t_{2}\right\} \geqslant 2$, we have $k-\ell+1 \geqslant 2$, thus by $1 / \sqrt{W} \prec \Psi$ and using Ward identity for the term $\bar{G}_{p_{n+1} y}$ and another $G$ hidden in $\partial_{j_{n+1} i_{n+1}}^{t_{1}} \mathcal{Q}_{y y}$ we have

$$
\begin{aligned}
(8.7) & \prec \frac{\Psi^{2}}{\sqrt{L}} \cdot\left(\frac{\Psi^{3}}{\sqrt{\eta}}+\frac{\Psi}{\sqrt{L \eta}}\right)^{n} \cdot \Psi \cdot\left(\lambda \Psi+\frac{\Psi}{\sqrt{L}}+\frac{\Psi^{3}}{\sqrt{\eta}}\right)^{\ell-2} \cdot \frac{1}{\eta}\left(\lambda+\frac{1}{\sqrt{L}}+\frac{\Psi^{3}}{\sqrt{\eta}}\right)^{2} \mathbb{E}\left|\mathcal{Q}_{y y}\right|^{2 p-n-\ell-1} \\
& \prec\left(\frac{\Psi^{3}}{\sqrt{\eta}}+\frac{\Psi}{\sqrt{L \eta}}+\lambda \Psi\right)^{n+\ell-2} \cdot\left(\frac{\Psi}{\eta^{1 / 6}} \lambda^{2 / 3}+\frac{\Psi^{3}}{\sqrt{\eta}}+\frac{\Psi}{\sqrt{L \eta}}\right)^{3} \mathbb{E}\left|\mathcal{Q}_{y y}\right|^{2 p-n-\ell-1} \\
& \prec\left(\frac{\Psi^{3}}{\sqrt{\eta}}+\frac{\Psi}{\sqrt{L \eta}}+\lambda \Psi+\frac{\Psi}{\eta^{1 / 6}} \lambda^{2 / 3}\right)^{n+\ell+1} \mathbb{E}\left|\mathcal{Q}_{y y}\right|^{2 p-n-\ell-1} .
\end{aligned}
$$


For $t_{1}=t_{2}=1$, we have $k-\ell+1 \geqslant 1$, thus by exploring (8.6) carefully and use Ward identity we have

$$
\begin{aligned}
(8.7) & \prec \frac{\Psi}{\sqrt{L}} \cdot\left(\frac{\Psi^{3}}{\sqrt{\eta}}+\frac{\Psi}{\sqrt{L \eta}}\right)^{n} \cdot \Psi \cdot\left(\lambda \Psi+\frac{\Psi}{\sqrt{L}}+\frac{\Psi^{3}}{\sqrt{\eta}}\right)^{\ell-2} \cdot\left(\frac{1}{\eta} \lambda^{2}+\frac{\Psi}{\eta} \frac{1}{L}+\frac{1}{\eta} \frac{\Psi^{6}}{\eta}\right) \cdot \mathbb{E}\left|\mathcal{Q}_{y y}\right|^{2 p-n-\ell-1} \\
& \prec\left(\frac{\Psi^{3}}{\sqrt{\eta}}+\frac{\Psi}{\sqrt{L \eta}}+\lambda \Psi+\frac{\Psi}{\eta^{1 / 6}} \lambda^{2 / 3}\right)^{n+\ell+1} \mathbb{E}\left|\mathcal{Q}_{y y}\right|^{2 p-n-\ell-1} .
\end{aligned}
$$

Thus for Case 1 we have

$$
(8.4) \prec \sum_{\ell=1}^{2 p-n-1}\left(\frac{\Psi^{3}}{\sqrt{\eta}}+\frac{\Psi}{\sqrt{L \eta}}+\lambda \Psi+\frac{\Psi}{\eta^{1 / 6}} \lambda^{2 / 3}\right)^{n+\ell+1} \mathbb{E}\left|\mathcal{Q}_{y y}\right|^{2 p-n-\ell-1} .
$$

Case 2. Suppose $\ell=1$. We have $1 / W^{(k-1) / 2} \prec \Psi$. By (8.5) and Ward identity we have

$$
\begin{aligned}
(8.4) & \prec \frac{\Psi}{\sqrt{L}}\left(\frac{\Psi^{3}}{\sqrt{\eta}}+\frac{\Psi}{\sqrt{L \eta}}\right)^{n} \cdot \Psi \cdot \mathbb{E}\left|\sum_{i_{n+1}, j_{n+1}} \sigma_{i_{n+1} j_{n+1}}^{(n+1)} \bar{G}_{i_{n+1} y} \partial_{j_{n+1} i_{n+1}}^{t} \mathcal{Q}_{y y}\right| \cdot\left|\mathcal{Q}_{y y}\right|^{\alpha+\beta-1} \\
& \prec\left(\frac{\Psi^{3}}{\sqrt{\eta}}+\frac{\Psi}{\sqrt{L \eta}}\right)^{n} \cdot \frac{\Psi^{2}}{\sqrt{L}} \cdot \frac{1}{\eta}\left(\lambda+\frac{1}{\sqrt{L}}+\frac{\Psi^{3}}{\sqrt{\eta}}\right) \cdot \mathbb{E}\left|\mathcal{Q}_{y y}\right|^{2 p-n-2} \\
& \prec\left(\frac{\Psi^{3}}{\sqrt{\eta}}+\frac{\Psi}{\sqrt{L \eta}}+\frac{\Psi}{L^{1 / 4} \eta^{1 / 2}} \lambda^{1 / 2}\right)^{n+2} \cdot \mathbb{E}\left|\mathcal{Q}_{y y}\right|^{2 p-n-2} .
\end{aligned}
$$

Case 3. Suppose $\ell=0$, and we see that similar as in Section 5.1, we can use Lemma 6.1 and shown that

$$
\begin{array}{r}
\frac{1}{L^{(n+1) / 2} W^{(k-1) / 2}} \sum_{i_{1}, j_{1}, \ldots, i_{n+1}, j_{n+1}} \sigma_{i_{1} j_{1}}^{(1)} \cdots \sigma_{i_{n+1} j_{n+1}}^{(n+1)} \partial_{j_{n+1} i_{n+1}}^{k}\left(G_{j_{1} y} \bar{G}_{i_{1} y} V_{1,2} \cdots V_{n-1, n} G_{j_{n+1} j_{n}} \bar{G}_{i_{n+1} y} G_{i_{n} y}\right) \\
\prec\left(\frac{\Psi^{3}}{\sqrt{\eta}}+\frac{\Psi}{\sqrt{L \eta}}\right)^{n+1},
\end{array}
$$

which implies

$$
(8.4) \prec\left(\frac{\Psi^{3}}{\sqrt{\eta}}+\frac{\Psi}{\sqrt{L \eta}}\right)^{n+1} \mathbb{E}\left|\mathcal{Q}_{y y}\right|^{2 p-n-1}
$$

Thus from Cases 1-3 we have

$$
\begin{aligned}
(8.4) & \prec \sum_{k=1}^{2 p-n}\left(\frac{\Psi^{3}}{\sqrt{\eta}}+\frac{\Psi}{\sqrt{L \eta}}+\lambda \Psi+\frac{\Psi}{\eta^{1 / 6}} \lambda^{2 / 3}+\frac{\Psi}{L^{1 / 4} \eta^{1 / 2}} \lambda^{1 / 2}\right)^{n+k} \mathbb{E}\left|\mathcal{Q}_{y y}\right|^{2 p-n-k} \\
& \prec \sum_{k=1}^{2 p}\left(\frac{\Psi^{3}}{\sqrt{\eta}}+\frac{\Psi}{\sqrt{L \eta}}\right)^{k / 3} \lambda^{2 k / 3} \mathbb{E}\left|\mathcal{Q}_{y y}\right|^{2 p-k}
\end{aligned}
$$

By estimating (6.9) in a similar fashion and using the steps in Section 6, we see that

$$
\mathbb{E}\left|\mathcal{Q}_{y y}\right|^{2 p} \prec \sum_{k=1}^{2 p}\left(\frac{\Psi^{3}}{\sqrt{\eta}}+\frac{\Psi}{\sqrt{L \eta}}\right)^{k / 3} \lambda^{2 k / 3} \mathbb{E}\left|\mathcal{Q}_{y y}\right|^{2 p-k} .
$$

Note that we can also estimate $\mathbb{E}\left|\mathcal{Q}_{x y}\right|^{2 p}$ exactly in the same way, i.e.

$$
\mathbb{E}\left|\mathcal{Q}_{x y}\right|^{2 p} \prec \sum_{k=1}^{2 p}\left(\frac{\Psi^{3}}{\sqrt{\eta}}+\frac{\Psi}{\sqrt{L \eta}}\right)^{k / 3} \lambda^{2 k / 3} \mathbb{E}\left|\mathcal{Q}_{x y}\right|^{2 p-k}
$$

whenever $\mathcal{Q}_{x y} \prec \lambda$ for all $x, y \in \mathbb{T}_{L}$. Hence, it suffices to apply Lemma 6.3 to conclude the proof. 
8.2. Non Gaussian terms in (7.11). In this section we want to estimate the non Gaussian terms in the cumulant expansion (7.13): using Lemma 3.2, we see that the non Gaussian terms yields the additional contributions $\mathcal{U}_{u a b}+$ $\left|R_{K+1}\right|$ to the right hand side of (7.13) where

$$
\mathcal{U}_{u a b}=\left|\mathbb{E} \sum_{i, j} \sigma_{u i} \sum_{\substack{w, t \geqslant 0 \\ w+t=2}}^{K} \frac{1}{w ! t !} \mathcal{C}^{(w, t+1)}\left(H_{j i}\right) \partial_{i j}^{t} \partial_{j i}^{w}\left[G_{a i} G_{j b}\left(Y_{u a b}\right)^{p-1}\left(\bar{Y}_{u a b}\right)^{p}\right]\right|, \quad \sigma \in \mathbb{S}
$$

and $R_{K+1}$ is given by (3.2). As in the previous section, from Lemma 3.4 (iii) in [12], we know that, given a large constant $D$, then one can choose $K=K(D)$ such that $R_{K+1}=O\left(L^{-D}\right)$.

Let us now focus on $\mathcal{U}_{\text {uab }}$ : since we are going to use very rough estimates where the complex conjugation does not play any role, in the following we will simplify the notation by neglecting it: i.e. we will replace $\bar{Y}_{u a b}$ by $Y_{u a b}$ and also $\partial_{j i}=\partial / \partial H_{j i}=\partial / \partial \bar{H}_{j i}$ by $\partial_{i j}=\partial / \partial H_{i j}$.

Using these notation conventions and (8.1), we can write

$$
\mathcal{U}_{u a b} \prec \sum_{n=2}^{K} U_{u a b}^{(n)}, \quad U_{u a b}^{(n)}:=\mathbb{E} \sum_{i, j}\left|\sigma_{u i}\right| S_{i j}^{(n+1) / 2}\left|\partial_{i j}^{n} G_{a i} G_{j b} Y_{u a b}^{2 p-1}\right| .
$$

Applying the Leibniz rule for derivatives we have

$$
\partial_{i j}^{n} G_{a i} G_{j b} Y_{u a b}^{2 p-1}=\sum_{r=0}^{n}\left(\partial_{i j}^{r} G_{a i} G_{j b}\right)\left(\partial_{i j}^{n-r} Y_{u a b}^{2 p-1}\right)
$$

thus a second application of the Leibniz rule allows us to estimate $U_{u a b}^{(n)}$ as a sum of contributions of the form

$$
\mathbb{E} \sum_{i, j}\left|\sigma_{u i}\right| S_{i j}^{(n+1) / 2}\left|\left(\partial_{i j}^{r} G_{a i} G_{j b}\right)\left(\prod_{t=1}^{h} \partial_{i j}^{\ell_{t}} Y_{u a b}\right) Y_{u a b}^{2 p-1-h}\right|
$$

where the sum runs over the integers $h=0, \ldots,(n-r) \wedge(2 p-1)$ and $\ell_{1}, \ldots, \ell_{h} \geqslant 1$ with $\ell_{1}+\cdots+\ell_{h}=n-r$. Let us split $U^{(n)}$ in three terms:

(a) the one corresponding to $r=0$ and $h=n$ (so that $\ell_{1}=\ell_{2}=\cdots=\ell_{n}=1$ ) is stochastically dominated by

$$
\text { (a) }:=\mathbb{E} \sum_{i, j} S_{i j}^{(n+1) / 2}\left|\sigma_{u i}\right|\left|G_{a i} G_{j b} \| \partial_{i j} Y_{u a b}\right|^{n}\left|Y_{u a b}\right|^{2 p-n-1},
$$

(b) the one where $r=0$ and $h \leqslant(n-1) \wedge(2 p-1)$ is dominated by

$$
\text { (b) }:=\mathbb{E} \sum_{i, j} S_{i j}^{(n+1) / 2}\left|\sigma_{u i} G_{a i} G_{j b}\right| \sum_{h=0}^{(n-1) \wedge(2 p-1)}\left|\prod_{t=1}^{h} \partial_{i j}^{\ell_{t}} Y_{u a b}\right|\left|Y_{u a b}\right|^{2 p-1-h},
$$

(c) the one where $r \geqslant 1$ is bounded by

$$
\text { (c) }:=\mathbb{E} \sum_{i, j} S_{i j}^{(n+1) / 2}\left|\sigma_{u i}\right| \sum_{r=1}^{n}\left|\partial_{i j}^{r} G_{a i} G_{j b}\right| \sum_{h=0}^{(n-r) \wedge(2 p-1)}\left|\prod_{t=1}^{h} \partial_{i j}^{\ell_{t}} Y_{u a b}\right|\left|Y_{u a b}\right|^{2 p-1-h} .
$$

Note that, since $K$ in (8.10) is big but fixed, we have that

$$
\mathcal{U}_{u a b} \prec(a)+(b)+(c) .
$$

We are now going to establish more explicit bounds for (a), (b) and (c). For (a), set the prior estimate $Y_{u a b} \prec \lambda$ with $\lambda \in\left[\Psi^{3}, L^{C}\right]$ for $a \neq b$. By using (7.14) and the trivial bound $Y_{u a b} \prec \Psi^{2}$, we get

$$
\partial_{i j} Y_{u a b} \prec \lambda \Psi+\lambda\left(\delta_{a i}+\delta_{b i}+\delta_{a j}+\delta_{b j}\right)+\Psi^{2}\left(\delta_{a i} \delta_{b j}+\delta_{a j} \delta_{b i}\right) .
$$


Therefore, from (8.15) and (8.13) we get that

$$
\begin{aligned}
(\mathrm{a}) & \prec\left(\Psi^{4 n+1}+\lambda^{n} \Psi^{n+2}+\Psi^{3 n+3}\right) \mathbb{E}\left|Y_{u a b}\right|^{2 p-n-1} \\
& \prec\left(\Psi^{3} \lambda^{2}\right)^{\frac{n+1}{3}} \mathbb{E}\left|Y_{u a b}\right|^{2 p-(n+1)},
\end{aligned}
$$

where in the last line we used that $n \geqslant 2$.

Let us now deal with (b) and (c): a simple induction shows that for any $r \geqslant 0$ and $r^{\prime} \geqslant 1$ and $\tau, \omega \in \mathbb{S}$

$$
\begin{aligned}
& \sum_{i, j} \tau_{u i} \omega_{i j}\left|\partial_{i j}^{r} G_{a i} G_{j b}\right| \prec \Psi^{2}, \\
& \sum_{i, j} \tau_{u i} \omega_{i j}\left|\partial_{i j}^{r} G_{a i} G_{j b}\right|\left(\delta_{a i}+\delta_{b i}+\delta_{a j}+\delta_{b j}+\delta_{i j}\right) \prec \Psi^{3}, \\
& \partial_{i j}^{r^{\prime}} Y_{u a b} \prec \Psi^{3}+\left(\delta_{a i}+\delta_{b i}+\delta_{a j}+\delta_{b j}+\delta_{i j}\right) \Psi^{2} .
\end{aligned}
$$

By (8.19), (8.17), (8.18) and (8.16) we get

$$
\begin{aligned}
(\mathrm{b}) & \prec \mathbb{E} \sum_{i, j} S_{i j}^{(n+1) / 2}\left|\sigma_{u i} G_{a i} G_{j b}\right| \sum_{h=0}^{(n-1) \wedge(2 p-1)}\left(\Psi^{3 h}+\left(\delta_{a i}+\delta_{b i}+\delta_{a j}+\delta_{b j}+\delta_{i j}\right) \Psi^{2 h}\right)\left|Y_{u a b}\right|^{2 p-1-h} \\
& \prec \Psi^{n+1} \sum_{h=0}^{2 p-1} \Psi^{3 h} \mathbb{E}\left|Y_{u a b}\right|^{2 p-1-h}+\Psi^{n+2} \sum_{h=0}^{(n-1) \wedge(2 p-1)} \Psi^{2 h} \mathbb{E}\left|Y_{u a b}\right|^{2 p-1-h} \\
& \prec \Psi^{n-2} \sum_{h^{\prime}=1}^{2 p} \Psi^{3 h^{\prime}} \mathbb{E}\left|Y_{u a b}\right|^{2 p-h^{\prime}}+\Psi^{n} \sum_{h^{\prime}=1}^{n \wedge 2 p} \Psi^{2 h^{\prime}} \mathbb{E}\left|Y_{u a b}\right|^{2 p-h^{\prime}} \prec \sum_{h^{\prime}=1}^{2 p} \Psi^{3 h^{\prime}} \mathbb{E}\left|Y_{u a b}\right|^{2 p-h^{\prime}}
\end{aligned}
$$

where in the last passage we used that $n \geqslant 2, n \geqslant h^{\prime}$ and $\Psi \leqslant 1$. The same argument shows that for (c) the same bound holds. Therefore, recalling (8.14) and the definition of $\lambda$, we have

$$
\mathcal{U}_{u a b} \prec\left(\Psi^{3} \lambda^{2}\right)^{\frac{n+1}{3}} \mathbb{E}\left|Y_{u a b}\right|^{2 p-(n+1)}+\sum_{l=1}^{2 p} \Psi^{3 l} \mathbb{E}\left|Y_{u a b}\right|^{2 p-l} \prec \sum_{l=1}^{2 p}\left(\Psi^{3} \lambda^{2}\right)^{l / 3} \mathbb{E}\left|Y_{u a b}\right|^{2 p-l} .
$$

This implies that in the general case the bound (7.16) becomes

$$
\mathbb{E}\left|Y_{u a b}\right|^{2 p} \prec \lambda \Psi^{3} \mathbb{E}\left|Y_{u a b}\right|^{2 p-2}+\sum_{l=1}^{2 p}\left(\Psi^{3} \lambda^{2}\right)^{l / 3} \mathbb{E}\left|Y_{u a b}\right|^{2 p-l} \prec \sum_{l=1}^{2 p}\left(\Psi^{3} \lambda^{2}\right)^{l / 3} \mathbb{E}\left|Y_{u a b}\right|^{2 p-l} .
$$

Applying Lemma 6.3 with $q=2 / 3, \vartheta=\lambda$ and $\varphi=\Psi^{3}$ concludes the proof for $a \neq b$. For $a=b$ the bound $Y_{\text {uaa }} \prec \Psi^{2}$ is trivial.

8.3. Non Gaussian terms in (7.12). First, we note that the same method used to treat the non Gaussian terms in $Y_{u a b}$ can be employed to show that the bounds (7.28) for $A_{a b c d}$ and (7.20) for $B_{a b}$ remain valid in the non Gaussian case.

Here we will focus on the additional terms arising from the expansion (7.17):

$$
\mathcal{U}_{a b}=\left|\mathbb{E} \sum_{i, j, k} \sigma_{a i} \tau_{b j} \sum_{w+t=2}^{K} \frac{1}{w ! t !} \mathcal{C}^{(w, t+1)}\left(H_{i k}\right) \partial_{i k}^{t} \partial_{k i}^{w}\left(G_{k j} G_{j i} Z_{a b}^{p-1} \bar{Z}_{a b}^{p}\right)\right|, \quad \sigma, \tau \in \mathbb{S} .
$$

By using the same notation simplification adopted for the non Gaussian terms of (7.11) in the previous Section 8.2 , we have

$$
\mathcal{U}_{a b} \prec \sum_{n=2}^{K} U_{a b}^{(n)}, \quad U_{a b}^{(n)}:=\mathbb{E} \sum_{i, j, k}\left|\sigma_{a i} \tau_{b j}\right| S_{k i}^{(n+1) / 2}\left|\partial_{k i}^{n} G_{k j} G_{j i} Z_{a b}^{2 p-1}\right|
$$


As before, a double application of the Leibniz rule implies that $U_{a b}^{(n)}$ is bounded by a sum of terms of the form

$$
\mathbb{E} \sum_{i, j, k}\left|\sigma_{a i} \tau_{b j}\right| S_{k i}^{(n+1) / 2}\left|\left(\partial_{k i}^{r} G_{k j} G_{j i}\right)\left(\prod_{t=1}^{h} \partial_{k i}^{\ell_{t}} Z_{a b}\right) Z_{a b}^{2 p-1-h}\right|
$$

where the sum runs over the integers $r=0, \ldots, n, h=0, \ldots,(n-r) \wedge(2 p-1)$ and $\ell_{1}, \ldots, \ell_{h} \geqslant 1$ with $\ell_{1}+\cdots+\ell_{h}=$ $n-r$. An induction argument involving (7.11) yields for $\ell \geqslant 1, r \geqslant 0$ and $\sigma, \tau, \omega \in \mathbb{S}$ that

$$
\begin{aligned}
& \mathbb{E} \sum_{i, j, k} \sigma_{a i} \tau_{b j} \omega_{k i}\left|\partial_{k i}^{r} G_{k j} G_{j i}\right| \prec \Psi^{2}, \\
& \partial_{k i}^{\ell} Z_{a b} \prec \Psi^{4} .
\end{aligned}
$$

Thus, by using (8.22), (8.23) and (8.24), we get

$$
\mathcal{U}_{a b} \prec \Psi^{n+1} \sum_{h=0}^{2 p-1} \Psi^{4 h} \mathbb{E}\left|Z_{a b}\right|^{2 p-(h+1)} \prec \Psi^{n-3} \sum_{h^{\prime}=0}^{2 p-1} \Psi^{4 h^{\prime}} \mathbb{E}\left|Z_{a b}\right|^{\left.2 p-h^{\prime}\right)} \prec \sum_{h^{\prime}=1}^{2 p} \Psi^{4 h^{\prime}} \mathbb{E}\left|Z_{a b}\right|^{2 p-h^{\prime}}
$$

where in the last passage we used that $n \geqslant 3$ and $\Psi \leqslant 1$. To complete the proof we examine in more detail what happens when $n=2$ which corresponds to the third order cumulant. We come back to the original expression (8.21) and we observe that the terms where $w+t=2$ are stochastically dominated by

$$
\begin{aligned}
& \left|\mathbb{E} \sum_{i, j, k} \sigma_{a i} \tau_{b j} \sum_{w+t=2} \frac{1}{w ! t !} \mathcal{C}^{(w, t+1)}\left(H_{i k}\right)\left(\partial_{i k}^{t} \partial_{k i}^{w} G_{k j} G_{j i}\right) Z_{a b}^{p-1} \bar{Z}_{a b}^{p}\right| \\
& +\left|\mathbb{E} \sum_{i, j, k} \sigma_{a i} \tau_{b j} \sum_{w+t=2} \frac{1}{w ! t !} \mathcal{C}^{(w, t+1)}\left(H_{i k}\right) G_{k j} G_{j i}\left(\partial_{k i}^{w} \partial_{i k}^{t} Z_{a b}^{p-1} \bar{Z}_{a b}^{p}\right)\right| \\
& +\left|\mathbb{E} \sum_{i, j, k} \sigma_{a i} \tau_{b j} \mathcal{C}^{(1,2)}\left(H_{i k}\right)\left(\partial_{k i} G_{k j} G_{j i}\right)\left(\partial_{i k} Z_{a b}^{p-1} \bar{Z}_{a b}^{p}\right)\right| \\
& +\left|\mathbb{E} \sum_{i, j, k} \sigma_{a i} \tau_{b j} \mathcal{C}^{(1,2)}\left(H_{i k}\right)\left(\partial_{i k} G_{k j} G_{j i}\right)\left(\partial_{k i} Z_{a b}^{p-1} \bar{Z}_{a b}^{p}\right)\right| \\
& +\left|\mathbb{E} \sum_{i, j, k} \sigma_{a i} \tau_{b j} \mathcal{C}^{(0,3)}\left(H_{i k}\right)\left(\partial_{i k} G_{k j} G_{j i}\right)\left(\partial_{i k} Z_{a b}^{p-1} \bar{Z}_{a b}^{p}\right)\right| \\
& +\left|\mathbb{E} \sum_{i, j, k} \sigma_{a i} \tau_{b j} \mathcal{C}^{(2,1)}\left(H_{i k}\right)\left(\partial_{k i} G_{k j} G_{j i}\right)\left(\partial_{k i} Z_{a b}^{p-1} \bar{Z}_{a b}^{p}\right)\right|=(\mathrm{a})+(\mathrm{b})+(\mathrm{c})
\end{aligned}
$$

where (c) denotes the sum of the last four terms on the left hand side of (8.26). Let us proceed term by term.

(a) We note that $\partial_{i k}^{t} \partial_{k i}^{w} G_{k j} G_{j i}$ can only generate of the form $G_{i i} G_{k k} G_{k j} G_{j i}$ and $G_{k i} G_{k k} G_{i j} G_{j i}$, up to switching $i$ to $k$. Therefore, plugging the first type of contribution in (a) and using (8.1) and (7.11) for the summation over $j$, we get

$$
\begin{aligned}
& \left|\mathbb{E} \sum_{i, j, k} \sigma_{a i} \tau_{b j} \sum_{w+t=2} \frac{1}{w ! t !} \mathcal{C}^{(w, t+1)}\left(H_{i k}\right) G_{i i} G_{k k} G_{k j} G_{j i} Z_{a b}^{p-1} \bar{Z}_{a b}^{p}\right| \\
& \prec \Psi \mathbb{E} \sum_{i, k}\left|\sigma_{a i} S_{i k}\right|\left(\Psi^{3}+\delta_{i k} \Psi^{2}\right)\left|Z_{a b}\right|^{2 p-1} \prec \Psi^{4} \mathbb{E}\left|Z_{a b}\right|^{2 p-1} .
\end{aligned}
$$

The second type of contribution has three non-diagonal entries of $G$, so we trivially get the same bound as for the former term. This implies that (a) $\prec \Psi^{4} \mathbb{E}\left|Z_{a b}\right|^{2 p-1}$. 
(b) By summing over $j$ and using (8.24) we get

$$
\begin{aligned}
\text { (b) } & \prec\left|\mathbb{E} \sum_{i, j, k} \sigma_{a i} \tau_{b j} \sum_{w+t=2} \frac{1}{w ! t !} \mathcal{C}^{(w, t+1)}\left(H_{i k}\right) G_{k j} G_{j i}\left(\partial_{k i}^{w} \partial_{i k}^{t} Z_{a b}^{p-1} \bar{Z}_{a b}^{p}\right)\right| \\
& \prec \Psi \mathbb{E} \sum_{i, k}\left|\sigma_{a i} S_{i k}\right|\left(\Psi^{3}+\delta_{i k} \Psi^{2}\right)\left|\partial_{k i}^{w} \partial_{i k}^{t} Z_{a b}^{p-1} \bar{Z}_{a b}^{p}\right| \\
& \prec \Psi \sum_{i, k}\left|\sigma_{a i} S_{i k}\right|\left(\Psi^{3}+\delta_{i k} \Psi^{2}\right)\left(\Psi^{4} \mathbb{E}\left|Z_{a b}\right|^{2 p-2}+\Psi^{8} \mathbb{E}\left|Z_{a b}\right|^{2 p-3}\right) \prec \Psi^{8} \mathbb{E}\left|Z_{a b}\right|^{2 p-2}+\Psi^{12} \mathbb{E}\left|Z_{a b}\right|^{2 p-3} .
\end{aligned}
$$

(c) The three terms in (c) have the same structure, so we will examine only the first one. According to (7.28), which is valid also in the non Gaussian case, the derivative of $Z$ is controlled as follows

$$
A_{a b c d}=\partial_{c d} Z_{a b} \prec \Psi^{5}+\delta_{c d} \Psi^{4} .
$$

Thus, from (8.1) we have

$$
(\mathrm{c}) \prec \Psi \mathbb{E} \sum_{i, j, k}\left|\sigma_{a i} \tau_{b j} S_{i k}\right|\left|G_{k k} G_{i j} G_{j i}+G_{k j} G_{i k} G_{i j}\right|\left|A_{a b k i}\right|\left|Z_{a b}\right|^{2 p-2} \prec \Psi^{8} \mathbb{E}\left|Z_{a b}\right|^{2 p-2} .
$$

By putting together all the contributions and invoking Lemma 6.3, one concludes the proof.

8.4. General complex case. Here we explain how our results extend to the case when

$$
\mathbb{E} H_{i j}^{2} \neq 0
$$

Since the non-Gaussian terms in this case are treated exactly as when $\mathbb{E} H_{i j}^{2}=0$, we will assume that $H_{i j}$ is Gaussian.

The bounds in Proposition 5.1 revolve around the self consistent equation trick implemented in Lemma 7.1, so we need to examine how (8.28) affects this lemma. From the cumulant expansion formula in Lemma 3.2 we see that equation (7.2) becomes

$$
z D_{a b c}=-\mathbb{E} \sum_{i} \sigma_{a i} \delta_{i b} G_{c i} \mathfrak{p}(G, \bar{G})+\mathbb{E} \sum_{i} \sigma_{a i}\left(S_{i j} \partial_{j i}+\mathcal{C}^{(2,0)}\left(H_{i j}\right) \partial_{i j}\right)\left(G_{j b} G_{c i} \mathfrak{p}(G, \bar{G})\right) .
$$

Note that $\mathcal{C}^{(2,0)}\left(H_{i j}\right)=\mathbb{E} H_{i j}^{2} \in \mathbb{S}$ since $\left|\mathcal{C}^{(2,0)}\left(H_{i j}\right)\right| \leqslant S_{i j}$ and $\partial_{i j} G_{j b} G_{c i}=-2 G_{j i} G_{j b} G_{c i}$ : this means that the additional term never generates any diagonal entry of $G$ and therefore it is always smaller than the contribution proportional to $\mathbb{E}\left|H_{i j}\right|^{2}=S_{i j}$.

As an example, let us write down in details the estimate of the additional terms for the estimate of the elementary chain $Y_{a b ; u}^{(1)} \equiv Y_{u a b}$ (Lemma 6.1). From Lemma 7.1 modified according to (8.29), we get

$$
\begin{aligned}
& \mathbb{E}\left|Y_{u a b}\right|^{2 p} \prec\left|\mathbb{E} \breve{\sigma}_{u a} G_{a b}\left(Y_{u a b}\right)^{p-1}\left(\bar{Y}_{u a b}\right)^{p}\right|+\left|\mathbb{E} \sum_{i, j} \breve{\sigma}_{u i} \omega_{i j} G_{a i} G_{j b} \partial_{j i}\left(Y_{u a b}^{p-1} \bar{Y}_{u a b}^{p}\right)\right| \\
& +\left|\mathbb{E} \sum_{i, j} \breve{\sigma}_{u i} S_{i j}\left(\left(G_{j j}-\mathfrak{m}\right) G_{a i} G_{i b}+G_{a j} G_{j b}\left(G_{i i}-\mathfrak{m}\right)\right) Y_{u a b}^{p-1} \bar{Y}_{u a b}^{p}\right| \\
& +\left|\mathbb{E} \sum_{i, j} \breve{\sigma}_{u i} \tau_{i j} G_{j i} G_{a i} G_{j b} Y_{u a b}^{p-1} \bar{Y}_{u a b}^{p}\right|
\end{aligned}
$$

where $\tau_{i j}=\mathcal{C}^{(2,0)}\left(H_{i j}\right), \omega=S+\tau$ and $\tau, \omega, \breve{\sigma} \in \mathbb{S}$. Note the last term on the right hand side of (8.30) is the only new additional contribution with respect to the former estimate (7.13). By using the hypothesis $\Lambda \prec \Psi$, we can easily control it:

$$
\mathbb{E} \sum_{i, j} \breve{\sigma}_{u i} \tau_{i j} G_{j i} G_{a i} G_{j b} Y_{u a b}^{p-1} \bar{Y}_{u a b}^{p} \prec \Psi^{3} \mathbb{E}\left|Y_{u a b}\right|^{2 p-1}
$$

Thus, (7.13) (and consequently (7.11)) actually holds true even when $\mathbb{E} H_{i j}^{2} \neq 0$. Analogous straightforward arguments show that also all the other bounds in Proposition 5.1 and lemmata 6.1, 7.2 and 7.3 remain valid. 


\section{High dimensions}

Fix $d \geqslant 2$ and recall that $N=L^{d}$ and $M \asymp W^{d}$. From Proposition 2.8 in [9] for $d \geqslant 2$ we have

$$
\Theta_{x y} \leqslant C \tilde{\Phi}^{2}
$$

where the explicit expression for $\Theta$ is given in Lemma 8.2 in [9] and

$$
\tilde{\Phi}^{2}:=\frac{1}{M}+\frac{1}{N \eta} .
$$

In this setting we can show the analogues of Theorem 2.2, Corollary 2.3 and Theorem 2.4 in [9].

Theorem 9.1 (High dimensions). Let $d \geqslant 2$ and assume (2.12).

(i) Suppose that

$$
L \ll W^{1+d / 2}, \quad \eta \gg \frac{L}{W^{1+d}}
$$

Then for $z \in \mathbf{S}$ we have

$$
\Lambda^{2} \prec \tilde{\Phi}^{2} .
$$

(ii) If $L \ll W^{1+\frac{d}{d+1}}$, then the eigenvectors of $H$ are completely delocalized in the sense of Proposition 7.1 in [9].

(iii) Assume that

$$
L \ll W^{1+d / 3}, \quad(W / L)^{2} \leqslant \eta \leqslant 1 .
$$

Then

$$
T_{x y}-\Theta_{x y} \prec \frac{1}{M^{3 / 2} \eta} .
$$

Moreover, the analogues of (2.21) and (2.22) hold with the explicit expression for $\Upsilon_{x y}$ given in Theorem 8.6 in [9].

Remark 9.2 (Comparison with the analogous results in [9]). Theorems 9.1 improves Theorem 8.4 and Theorem 8.6 in [9]. In fact, in Theorem 8.4 [9] it is assumed that $L \ll W^{1+d / 4}$ and $\eta \gg \frac{L^{2}}{W^{d+2}}$, in Theorem 8.6 that $L \ll W^{1+d / 4}$.

Furthermore, in Corollary 8.5 in [9] the stated condition, i.e. $L \ll W^{1+d / 4}$, is wrong: it should be $L \ll W^{1+\frac{d}{d+2}}$ because one must have that $\frac{L^{2}}{W^{d+2}} \ll \eta \leqslant N / M$ in order to apply Proposition 7.1. Thus Theorem 9.1(iii) improves also Corollary 8.5 in [9].

Proof. (i) For $d>1$ we treat the error term $\tilde{\mathcal{E}}$ of equation (1.1) very similarly to what we did in Proposition 4.1 for $d=1$. We define the analogue of the matrix $Q$ as $Q^{(\alpha)}$ such that $\widehat{q}^{(\alpha)}(p)=1-\chi\left(p W^{1-\alpha} L^{\alpha}\right)$ for $p \in \mathbb{T}_{L}^{d}$, where $q_{x}^{(\alpha)}=Q_{x 0}^{(\alpha)}$ for $x \in \mathbb{T}_{L}^{d}$ and $\chi$ is a smooth bump function as defined in the proof of Proposition 4.1. Here we set $\alpha \in[0,1)$ since for $\alpha \geqslant 1, \chi\left(p W^{1-\alpha} L^{\alpha}\right)=0$ for $p \neq 0$, thus we would be back to the old analysis performed in [9]. We will tune $\alpha$ in order to get the optimal conditions for the local law.

Let $w_{x}:=(\Pi \mathcal{E})_{x y}$, then

$$
\sup _{x, y}\left|\tilde{\mathcal{E}}_{x y}\right| \prec \sup _{y}\left\|\frac{Q_{\alpha}}{I-|\mathfrak{m}|^{2} S} \mathbf{w}\right\|_{\infty}+\sup _{y}\left\|\frac{I-Q_{\alpha}}{I-|\mathfrak{m}|^{2} S} \mathbf{w}\right\|_{\infty}
$$

where

$$
\begin{aligned}
& \sup _{y}\left\|\frac{I-Q_{\alpha}}{I-|\mathfrak{m}|^{2} S} \mathbf{w}\right\|_{\infty}=\sup _{y}\left\|\sum_{p \in\left(\mathbb{T}_{L}^{d}\right)^{*}, p \neq 0} \frac{\chi\left(p W^{1+\alpha}\right)}{1-|\mathfrak{m}|^{2} \widehat{s}(p)} \mathbf{e}(p)\langle\mathbf{e}(p), \mathcal{E}\rangle_{y}\right\|_{\infty} \\
& \prec \frac{1}{\sqrt{N}} \min \left\{\frac{1}{\eta},\left(\frac{L}{W}\right)^{2}\right\}\left(\frac{L}{W}\right)^{(d-2)(1-\alpha)} \sup _{y} \sup _{p \neq 0}\left|\langle\mathbf{e}(p), \mathcal{E}\rangle_{y}\right|
\end{aligned}
$$


and

$$
\sup _{y}\left\|\frac{Q_{\alpha}}{1-|\mathfrak{m}|^{2} S} \mathbf{w}\right\|_{\infty} \prec \frac{1}{\eta+(W / L)^{2 \alpha}} \sup _{x, y}\left|\mathcal{E}_{x y}\right|
$$

Hence, for $\alpha<1$

$$
\sup _{x, y}\left|\tilde{\mathcal{E}}_{x y}\right| \prec \frac{1}{\sqrt{N}} \min \left\{\frac{1}{\eta},\left(\frac{L}{W}\right)^{2}\right\}\left(\frac{L}{W}\right)^{(d-2)(1-\alpha)} \sup _{y} \sup _{p \neq 0}\left|\langle\mathbf{e}(p), \mathcal{E}\rangle_{y}\right|+\frac{1}{\eta+(W / L)^{2 \alpha}} \sup _{x, y}\left|\mathcal{E}_{x y}\right|,
$$

while for $\alpha \geqslant 1$ we get the same bound as in [9], i.e.

$$
\sup _{x, y}\left|\tilde{\mathcal{E}}_{x y}\right| \prec \frac{1}{\eta+(W / L)^{2}} \sup _{x, y}\left|\mathcal{E}_{x y}\right| .
$$

Moreover, from [9] we know that

$$
\sup _{x, y}\left|\mathcal{E}_{x y}\right| \prec \Psi^{4}+\Psi^{2} M^{-1 / 2} .
$$

Note that (9.7) is derived by using the fluctuation averaging bounds in [7], but we believe that it can be obtained also by the cumulant expansion method by performing nested expansions of $\mathcal{P}_{x y}$ and $\mathcal{R}_{x y}$ defined in (5.2) and (5.3).

By combining (9.7) with the (5.8) and (5.10) in Proposition 5.1 we get

$$
\sup _{x, y}\left|\tilde{\mathcal{E}}_{x y}\right| \prec\left(\frac{L}{W}\right)^{2 \alpha} \Psi^{2}\left[\left(\frac{N}{M}\right)^{1-\alpha}\left(\frac{1}{N \eta}+\frac{\Psi^{-1}}{N \sqrt{\eta}}+\frac{\Psi}{\sqrt{N \eta}}\right)+\Psi^{2}+M^{-1 / 2}\right] .
$$

Recall that initially, thanks to Lemma $3.4, \Psi^{2}=(M \eta)^{-1}$ and that $\Psi^{-2} \leqslant N \eta+M$. In order to apply Lemma 5.3 , we need to have

$$
L \ll W^{1+\frac{d}{4 \alpha+d(1-2 \alpha)}}, \quad \eta \gg \frac{L^{2 \alpha+d / 2-d \alpha}}{W^{2 \alpha+3 d / 2-d \alpha}}, \quad \eta \gg \frac{L^{2 \alpha}}{W^{2 \alpha+d}}, \quad L \ll W^{1+d / 4 \alpha} .
$$

By comparing the above conditions, it is easy to see that the optimal conditions are attained for $\alpha=1 / 2$, i.e.

$$
L \ll W^{1+d / 2}, \quad \eta \gg \frac{L}{W^{1+d}} .
$$

Observe that from (9.5) we see that for $d=2$ we can choose any $\alpha \in[0,1 / 2]$ because the sum over the moments is only logarithmically divergent:

$$
\sum_{\substack{p \in\left(\mathbb{T}_{L}^{2}\right)^{*} \\ p \neq 0}} \frac{\chi\left(p W^{1+\alpha}\right)}{1-|\mathfrak{m}|^{2} \widehat{s}(p)} \leqslant C \min \left\{\frac{1}{\eta}, \frac{L^{2}}{W^{2}}\right\} \sum_{\substack{j \in \mathbb{Z}^{2} \\ 0<|j| \leqslant L / W^{1+\alpha}}}|j|^{-2}=\min \left\{\frac{1}{\eta}, \frac{L^{2}}{W^{2}}\right\} O\left(\log \frac{L}{W^{1+\alpha}}\right) .
$$

(ii) From (2.17) and (i) we know that for the eigenvector delocalization we need $\eta\left\langle M / N\right.$ and $\eta \gg L / W^{1+d}$, which is true when $L \ll W^{1+\frac{d}{d+1}}$.

(iii) From (i) we know that $\Lambda^{2} \prec M^{-1}+(N \eta)^{-1}$ when $L \ll W^{1+d / 2}$ and $\eta \gg L / W^{1+d}$. Here we want $\eta \geqslant(W / L)^{2}$, therefore, in order to use (i), we need to require

$$
\frac{L}{W^{1+d}} \ll \frac{W^{2}}{L^{2}},
$$

i.e. $L \ll W^{1+d / 3}$. Note also that $\eta \geqslant(W / L)^{2}$ implies $\eta \geqslant M / N$ for $d \geqslant 2$, thus $\Lambda^{2} \prec M^{-1}$. This means that (9.5) holds with $\Psi=M^{-1 / 2}$. In this setting it is easy to check from (9.5) and (9.6) that (9.3) and the analogues of (2.21) and (2.22) are valid for any $\alpha \geqslant 1 / 2$. 


\section{A Proof of Lemma 5.2}

We recall that Lemma 5.2 basically coincides with Corollary 5.4 in [9]. Here we give a proof which does not rely on the averaging fluctuations estimate in [7].

We will assume that $H$ is Hermitian with Gaussian entries and such that $\mathbb{E} H_{i j}^{2}=0$, but the result holds also in the general complex case and the additional terms are treated as we saw in Section 8.

To get the desired bounds, we consider the expectation $\mathcal{F}_{a b}:=\mathbb{E}\left|F_{a b}\right|^{2 p}$ where $F_{a b}:=G_{a b}-\mathfrak{m} \delta_{a b}$ and $p$ is an arbitrary strictly positive integer. The cumulant expansion yields

$$
\begin{aligned}
& z \mathcal{F}_{a b}=\mathbb{E}\left(z G_{a b}-z \mathfrak{m} \delta_{a b}\right) F_{a b}^{p-1} \bar{F}_{a b}^{p}=\mathbb{E}\left(\sum_{i} H_{a i} G_{i b}-(1+z \mathfrak{m}) \delta_{a b}\right) F_{a b}^{p-1} \bar{F}_{a b}^{p} \\
& =\mathfrak{m}^{2} \delta_{a b} \mathbb{E} F_{a b}^{p-1} \bar{F}_{a b}^{p}-\mathbb{E} \sum_{i} S_{a i} \partial_{i a} G_{i b} F_{a b}^{p-1} \bar{F}_{a b}^{p} \\
& =\mathfrak{m}^{2} \delta_{a b} \mathbb{E} F_{a b}^{p-1} \bar{F}_{a b}^{p}-\mathbb{E} \sum_{i} S_{a i} G_{i i} G_{a b} F_{a b}^{p-1} \bar{F}_{a b}^{p}+\mathbb{E} \sum_{i} S_{a i} G_{i b} \partial_{i a} F_{a b}^{p-1} \bar{F}_{a b}^{p}
\end{aligned}
$$

where we used (2.10). Using the trivial identities $G_{i i}=G_{i i}-\mathfrak{m}+\mathfrak{m}$ and $G_{a b}=F_{a b}+\mathfrak{m} \delta_{a b}$, we get

$$
\begin{aligned}
(z+\mathfrak{m}) \mathcal{F}_{a b}= & -\mathbb{E} \sum_{i} S_{a i}\left(G_{i i}-\mathfrak{m}\right) F_{a b} F_{a b}^{p-1} \bar{F}_{a b}^{p}-\mathfrak{m} \delta_{a b} \mathbb{E} \sum_{i} S_{a i}\left(G_{i i}-\mathfrak{m}\right) F_{a b}^{p-1} \bar{F}_{a b}^{p} \\
& +\mathbb{E} \sum_{i} S_{a i} G_{i b} \partial_{i a} F_{a b}^{p-1} \bar{F}_{a b}^{p} .
\end{aligned}
$$

Using (6.5), (2.10) and that $\left|F_{a b}\right| \leqslant \Lambda \prec \Psi$, one obtains

$$
\mathcal{F}_{a b}=O_{\prec}\left(\Psi^{2} \mathbb{E}\left|F_{a b}\right|^{2 p-1}\right)+O_{\prec}\left(\left|\mathbb{E} \sum_{i} S_{a i} G_{i b} \partial_{i a} F_{a b}^{p-1} \bar{F}_{a b}^{p}\right|\right) .
$$

Let us examine the second term on the right hand side of (A.1): we note that

$$
\partial_{i a} F_{a b}=-G_{a i} G_{a b}, \quad \partial_{i a} \bar{F}_{a b}=-\bar{G}_{a a} \bar{G}_{i b},
$$

therefore (A.1) becomes

$$
\begin{aligned}
\mathcal{F}_{a b}= & O_{\prec}\left(\Psi^{2} \mathbb{E}\left|F_{a b}\right|^{2 p-1}\right)+O_{\prec}\left(\left|\mathbb{E} \sum_{i} S_{a i} G_{i b} G_{a i} G_{a b} F_{a b}^{p-2} \bar{F}_{a b}^{p}\right|\right) \\
& +O_{\prec}\left(\left.\left|\mathbb{E} \sum_{i} S_{a i}\right| G_{i b}\right|^{2} \bar{G}_{a a} F_{a b}^{p-1} \bar{F}_{a b}^{p-1} \mid\right) .
\end{aligned}
$$

In the second term on the right hand side of (A.2), when $a \neq b$ we can use (7.11) so that

$$
\mathcal{F}_{a b} \prec \Psi^{2} \mathbb{E}\left|F_{a b}\right|^{2 p-1}+\mathbb{E}\left(\Psi^{4}+T_{a b}\right)\left|F_{a b}\right|^{2 p-2},
$$

while, when $a=b$, the trivial inequality $|x y| \leqslant\left(|x|^{2}+|y|^{2}\right) / 2$ yields

$$
\sum_{i} S_{a i} G_{i b} G_{a i} \prec \sum_{i} S_{a i}\left(\left|G_{a i}\right|^{2}+\left|G_{i a}\right|^{2}\right)=T_{a a}+T_{a a}^{\prime}
$$

so that

$$
\mathcal{F}_{a a} \prec \Psi^{2} \mathbb{E}\left|F_{a a}\right|^{2 p-1}+\mathbb{E}\left(T_{a a}+T_{a a}^{\prime}\right)\left|F_{a a}\right|^{2 p-2}
$$

Thus, we finally have

$$
\mathcal{F}_{a b} \prec \Psi^{2} \mathbb{E}\left|F_{a b}\right|^{2 p-1}+\left(\Psi^{4}+\Omega_{a b}\right) \mathbb{E}\left|F_{a b}\right|^{2 p-2} \prec\left(\Psi^{2}+\Omega_{a b}\right) \mathbb{E}\left|F_{a b}\right|^{2 p-1}+\left(\Psi^{4}+\Omega_{a b}^{2}\right) \mathbb{E}\left|F_{a b}\right|^{2 p-2} .
$$

Lemma 6.3 completes the proof.

Remark A.1. The estimate in Lemma 5.2 cannot be improved in the sense that $F_{a b}$ will always be bounded at least by $T_{a b}=\sum_{i} S_{a i}\left|G_{i b}\right|^{2}$ because $T_{a b}$ appears explicitly in the third term on the right hand side of (A.2). 


\section{References}

[1] P.W. Anderson, Absence of diffusion in certain random lattices, Phys. Rev. 109 (1958), 1492.

[2] P. Bourgade, F. Yang, H.-T. Yau, and J. Yin, Random band matrices in the delocalized phase, II: Generalized resolvent estimates. J. Stat. Phys. 174 (2019), pp 11891221.

[3] P. Bourgade, H.-T. Yau and J. Yin, Random band matrices in the delocalized phase, I: Quantum unique ergodicity and universality. Preprint arXiv:1807.01559.

[4] A. Boutet de Monvel and A. Khorunzhy, Asymptotic distribution of smoothed eigenvalue density. II. Wigner random matrices, Random Oper. and Stoch. Equ. 7 (1999), 149-168.

[5] L. Erdős and A. Knowles, Quantum diffusion and delocalization for band matrices with general distribution, Ann. H. Poincaré 12 (2011), 1227-1319.

[6] L. Erdős and A. Knowles, Quantum diffusion and eigenfunction delocalization in a random band matrix model, Comm. Math. Phys. 303 (2011), 509-554.

[7] L. Erdős, A. Knowles, and H.-T. Yau, Averaging fluctuations in resolvents of random band matrices, Ann. H. Poincaré 14 (2013), 1837-1925.

[8] L. Erdős, A. Knowles, H.T. Yau, and J. Yin, The local semicircle law for a general class of random matrices, Elect. J. Prob. 18 (2013), no. 59, 58 pages.

[9] L. Erdős, A. Knowles, H.-T. Yau, and J. Yin, Delocalization and diffusion profile for random band matrices , Comm. Math. Phys. 323 (2013), 367-416.

[10] L. Erdős, H.-T. Yau and J. Yin, Bulk universality for generalized Wigner matrices, J. Probab. Theory Relat. Fields 154(1) (2012) 341-407.

[11] Y.V. Fyodorov and A.D. Mirlin, Scaling properties of localization in random band matrices: a $\sigma$-model approach, Phys. Rev. Lett. 67 (1991), 2405-2409.

[12] Y. He, A. Knowles and R. Rosenthal Isotropic self-consistent equations for mean-field random matrices, to appear in Prob. Theor. Rel. Fields.

[13] Y. He and A. Knowles, Mesoscopic eigenvalue statistics of Wigner matrices, Ann. Appl. Prob. 27 (2017), $1510-1550$.

[14] A.M. Khorunzhy, B.A. Khoruzhenko, and L.A. Pastur, Asymptotic properties of large random matrices with independent entries, J. Math. Phys. 37 (1996), 5033-5060.

[15] J. Schenker, Eigenvector localization for random band matrices with power law band width, Comm. Math. Phys. 290 (2009), 1065-1097.

[16] T. Spencer, SUSY statistical mechanics and random band matrices, lecture notes.

[17] T. Spencer, Random banded and sparse matrices (Chapter 23), "Oxford Handbook of Random Matrix Theory" edited by G. Akemann, J. Baik, and P. Di Francesco (2011).

[18] E.P. Wigner, Characteristic vectors of bordered matrices with infinite dimensions, Ann. Math. 62 (1955), $548-564$.

[19] F. Yang and J. Yin, Random band matrices in the delocalized phase, III: Averaging fluctuations. Preprint arXiv:1807.02447. 